\title{
Multiwavelength optical observations of chromospherically active binary systems
}

\author{
III. High resolution echelle spectra from $\mathrm{Ca}$ II $\mathrm{H} \& \mathrm{~K}$ to $\mathrm{Ca}$ II IRT ${ }^{\star, \star \star}$ \\ D. Montes ${ }^{1,2}$, M.J. Fernández-Figueroa ${ }^{1}$, E. De Castro ${ }^{1}$, M. Cornide ${ }^{1}$, A. Latorre ${ }^{1}$, and J. Sanz-Forcada ${ }^{1,3}$ \\ 1 Departamento de Astrofísica, Facultad de Ciencias Físicas, Universidad Complutense de Madrid, E-28040 Madrid, Spain \\ 2 Guest observer at McDonald Observatory, Texas, U.S.A. \\ 3 Harvard-Smithsonian Center for Astrophysics, 60 Garden St., Cambridge, MA 02138, U.S.A.
}

Received April 10; accepted July 3, 2000

\begin{abstract}
This is the third paper of a series aimed at studying the chromosphere of active binary systems using the information provided for several optical spectroscopic features. High resolution echelle spectra including all the optical chromospheric activity indicators from the Ca II $\mathrm{H} \& \mathrm{~K}$ to $\mathrm{Ca}$ II IRT lines are analysed here for 16 systems. The chromospheric contribution in these lines has been determined using the spectral subtraction technique. Very broad wings have been found in the subtracted $\mathrm{H} \alpha$ profile of the very active star HU Vir. These profiles are well matched using a two-component Gaussian fit (narrow and broad) and the broad component can be interpreted as arising from microflaring. Red-shifted absorption features in the $\mathrm{H} \alpha$ line have been detected in several systems and excess emission in the blue wing of FG UMa was also detected. These features indicate that several dynamical processes, or a combination of them, may be involved. Using the $E_{\mathrm{H} \alpha} / E_{\mathrm{H} \beta}$ ratio as a diagnostic we have detected prominence-like extended material viewed off the limb in many stars of the sample, and prominences viewed against the disk at some orbital phases in the dwarfs OU Gem and BF Lyn. The He $\mathrm{I}_{3}$ line has been detected as an absorption feature in mainly all the giants of the sample.
\end{abstract}

Send offprint requests to: D. Montes

* Based on observations made with the Isaac Newton Telescope (INT) operated on the island of La Palma by the Isaac Newton Group in the Spanish Observatorio del Roque de Los Muchachos of the Instituto de Astrofísica de Canarias, and with the Nordic Optical Telescope (NOT), operated on the island of La Palma jointly by Denmark, Finland, Iceland, Norway and Sweden, in the Spanish Observatorio del Roque de los Muchachos of the Instituto de Astrofísica de Canarias.

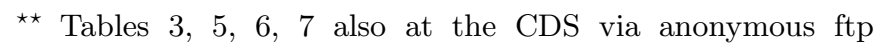
to cdsarc.u-strasbg.fr (130.79.128.5) or via http://cdsweb.ustrasbg.fr/Abstract.html

Correspondence to: e-mail: dmg@astrax.fis.ucm.es
Total filling-in of the He I $\mathrm{D}_{3}$, probably due to microflaring activity, is observed in HU Vir. Self-absorption with red asymmetry is detected in the $\mathrm{Ca}$ II $\mathrm{H} \& \mathrm{~K}$ lines of the giants 12 Cam, FG UMa and BM CVn. All the stars analysed show clear filled-in Ca II IRT lines or even notable emission reversal. The small values of the $E_{8542} / E_{8498}$ ratio we have found indicate Ca II IRT emission arises from plage-like regions. Orbital phase modulation of the chromospheric emission has been detected in some systems, in the case of $\mathrm{HU}$ Vir evidence of an active longitude area has been found.

Key words: stars: activity — stars: binaries: close — stars: binaries: spectroscopic — stars: chromospheres — stars: late-type

\section{Introduction}

This paper is a continuation of our ongoing project of multiwavelength optical observations aimed at studying the chromosphere of active binary systems using the information provided for several optical spectroscopic features that are formed at different heights in the chromosphere. In Paper I (Montes et al. 1997) we focussed our study on the analysis of the extensively used $\mathrm{H} \alpha$ chromospheric activity indicator together with simultaneous observations of the less studied He I $\mathrm{D}_{3}$ and $\mathrm{Na} \mathrm{I}_{1}, \mathrm{D}_{2}$ spectral features in a sample of 18 northern active binary systems. In Paper II (Montes et al. 1998a) the $\mathrm{H} \alpha, \mathrm{H} \beta, \mathrm{Na}$ I $\mathrm{D}_{1}, \mathrm{D}_{2}$, He I $\mathrm{D}_{3}, \mathrm{Mg}$ I b triplet, Ca II H \& $\mathrm{K}$ and $\mathrm{Ca}$ II infrared triplet lines (Ca II IRT) of the RS CVn system EZ Pegasi were studied at different orbital phases, also including a high resolution echelle spectrum. 
Table 1. Observing $\log$

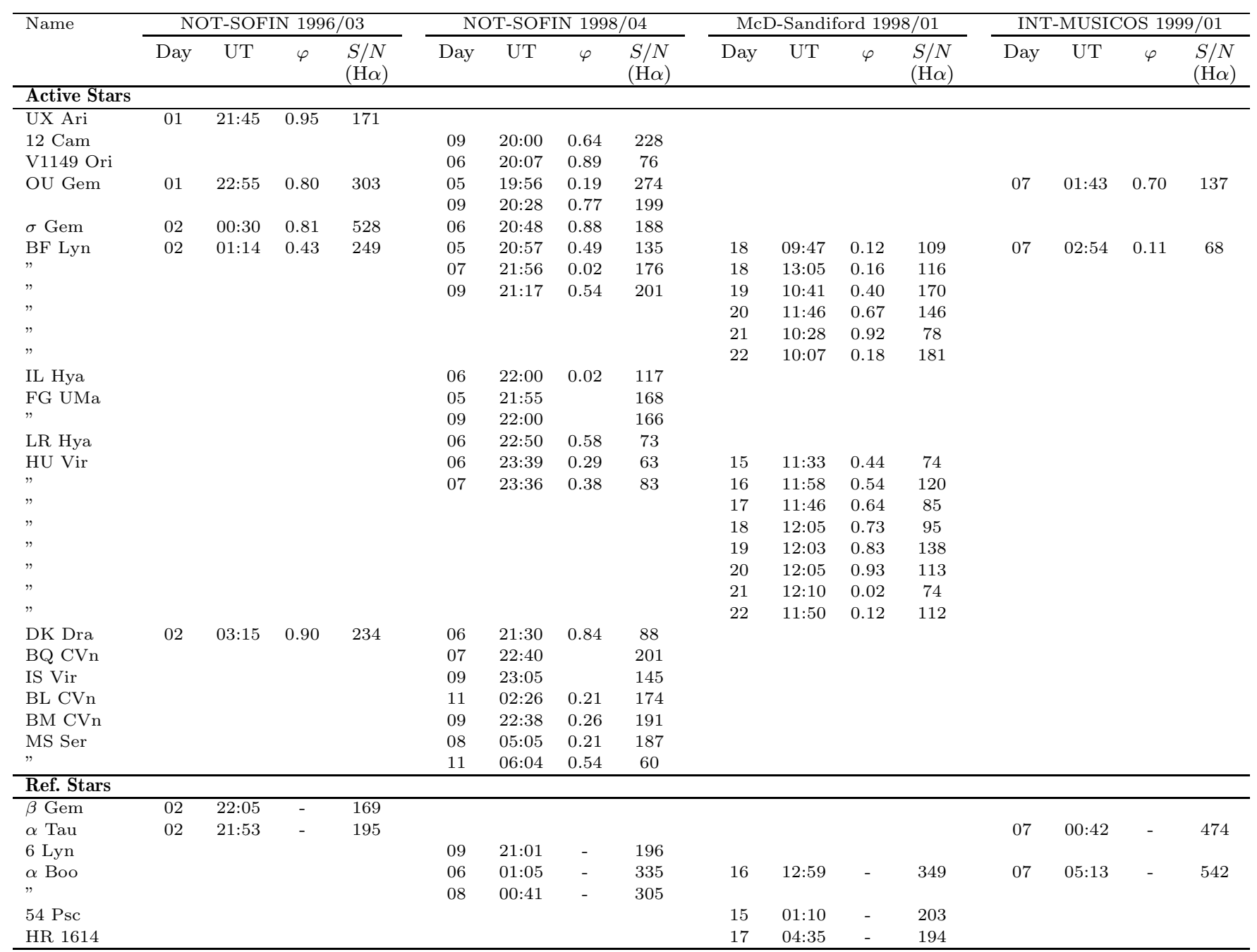

As shown in Papers I and II, with the simultaneous analysis of the different optical chromospheric activity indicators and using the spectral subtraction technique, it is possible to study in detail the chromosphere, discriminating between the different structures: plages, prominences, flares and microflares (see recent studies by Gunn \& Doyle 1997; Gunn et al. 1997; Lázaro \& Arévalo 1997; Arévalo \& Lázaro 1999; Hall \& Wolovitz 1998; Montes et al. 1998b; Montes \& Ramsey 1998, 1999; Montes et al. 1999; Eibe et al. 1999; Oliveira \& Foing 1999; Berdyugina et al. 1999).

In this paper we present high resolution echelle spectra of 16 systems selected from "A Catalog of Chromospherically Active Binary Stars (second edition)" (Strassmeier et al. 1993, hereafter CABS). The spectra were taken during four observing runs (from 1996 to 1999) and include all the optical chromospheric activity indicators from the $\mathrm{Ca}$ II $\mathrm{H} \& \mathrm{~K}$ to $\mathrm{Ca}$ II IRT lines. Preliminary results of some of the systems included here can be found in Sanz-Forcada et al. $(1998,1999)$, Montes et al. (1998c, 2000b), Latorre et al. (1999, 2000).
In Sect. 2 we give the details of our observations and data reduction. In Sect. 3 we describe the spectroscopic features analysed in this paper: the different chromospheric activity indicators and the $\mathrm{Li}$ I $\lambda 6707.8$ line. Individual results about stellar parameters and the behaviour of the chromospheric excess emission in each system is reported in Sect. 4. Finally, in Sect. 5 the discussion and conclusions are given.

\section{Observations and data reduction}

The spectroscopic echelle observations of the chromospherically active binaries analysed in this paper were obtained during four observing runs. Two of them were carried out in 1-2 March 1996 (NOT96 hereafter) and in 510 April 1998 (NOT98 hereafter), using the $2.56 \mathrm{~m}$ Nordic Optical Telescope (NOT) located at the Observatorio del Roque de Los Muchachos (La Palma, Spain). The Soviet Finnish High Resolution Echelle Spectrograph (SOFIN) was used with an echelle grating (79 grooves $/ \mathrm{mm}$ ), 
Table 2. Stellar parameters

\begin{tabular}{|c|c|c|c|c|c|c|c|c|c|c|}
\hline HD & Name & $\overline{T_{\mathrm{sp}}}$ & SB & $\begin{array}{c}R \\
\left(R_{\odot}\right) \\
\end{array}$ & $V-R$ & $B-V$ & $\begin{array}{l}T_{\text {conj }} \\
\text { (HJD) }\end{array}$ & $\begin{array}{l}\begin{array}{l}P_{\text {orb }} \\
\text { (days) }\end{array} \\
\end{array}$ & $\begin{array}{l}P_{\text {rot }} \\
\text { (days) }\end{array}$ & $\begin{array}{c}V \sin i \\
\left(\mathrm{~km} \mathrm{~s}^{-1}\right)\end{array}$ \\
\hline \multicolumn{11}{|c|}{ Active Stars } \\
\hline 21242 & UX Ari & G5V/K0IV & 2 & $0.93 />4.7$ & $0.54 / 0.70$ & 0.91 & 2450415.0647 & 6.437876 & $\approx P_{\mathrm{orb}}$ & $6 / 37$ \\
\hline 32357 & $12 \mathrm{Cam}$ & KOIII & 1 & $\geq 16$ & {$[0.77]$} & 1.12 & 2448111.1 & 80.898 & 81.23 & 11.3 \\
\hline 37824 & V1149 Ori & K1III & 1 & $/ \geq 11$ & 0.90 & 1.14 & 2444325.93 & 53.580 & 54.1 & 11 \\
\hline 45088 & OU Gem & $\mathrm{K} 3 \mathrm{~V} / \mathrm{K} 5 \mathrm{~V}$ & 2 & $0.77 / 0.72$ & {$[0.82 / 0.99]$} & {$[0.96 / 1.15]$} & 2443846.2 & 6.991868 & 7.36 & $5.6 / 5.6$ \\
\hline 62044 & $\sigma$ Gem & K1III & 1 & $>10.5$ & 0.92 & 1.122 & 2447227.22 & 19.604471 & 19.601562 & 27 \\
\hline 80715 & BF Lyn & $\mathrm{K} 2 \mathrm{~V} /[\mathrm{dK}]$ & 2 & $>0.78 />0.78$ & {$[0.74 /]$} & 0.99 & 2445802.472 & 3.80406 & $\approx P_{\mathrm{orb}}$ & $10 / 10$ \\
\hline 81410 & IL Hya & G8V/K0III-IV & 2 & $1.0 / 8.1$ & 0.79 & 1.02 & 2447103.1723 & 12.904982 & 12.730 & 26.5 \\
\hline 89546 & FG UMa & G8IV & 1 & $>6.4$ & 0.64 & 1.004 & & 21.300 & 21.50 & 15 \\
\hline 91816 & LR Hya & $\mathrm{K} 0 \mathrm{~V} / \mathrm{K} 0 \mathrm{~V}$ & 2 & $\geq 0.8 / \geq 0.8$ & {$[0.64 / 0.64]$} & {$[0.81 / 0.81]$} & 2446539.89 & 6.86569 & 3.1448 & $6 / 6$ \\
\hline 106225 & HU Vir & K0III-IV & 1 & $>5.7$ & {$[0.705]$} & 0.974 & 2450195.909 & 10.3876 & 10.42 & 31.3 \\
\hline 106677 & DK Dra & K1III/K1III & 2 & $\geq 13 / \geq 13$ & {$[0.81 / 0.81]$} & {$[1.07 / 1.07]$} & 2443445.95 & 64.44 & 63.75 & $10 / 10$ \\
\hline 112859 & BQ CVn & F/KOIII & 2 & $/>6.2$ & {$[0.77]$} & 0.93 & & 18.700 & 18.50 & $<6 / 17$ \\
\hline 113816 & IS Vir & KOIII & 1 & $>2.3$ & 0.93 & 1.15 & & 23.700 & 23.50 & 6 \\
\hline 115781 & $\mathrm{BL} \mathrm{CVn}$ & G-KIV/KOIII & 2 & $/ 14.8$ & {$[/ 0.77]$} & 1.14 & 2445284.47 & 18.6917 & $\approx P_{\text {orb }}$ & $7 / 35$ \\
\hline 116204 & BM CVn & K1III & 1 & $\geq 6$ & {$[0.81]$} & 1.16 & 24445256.776 & 20.625 & 20.6 & 15 \\
\hline 143313 & MS Ser & G8V/K2IV & 2 & $1.0 / 3.5$ & {$[0.58 / 0.79]$} & $0.94 / 1.23$ & 2442616.142 & 9.01490 & 9.60 & $7 / 15$ \\
\hline \multicolumn{11}{|c|}{ Ref. Stars } \\
\hline 62509 & $\beta$ Gem & K0III & & & & & & & & \\
\hline 29139 & $\alpha$ Tau & K5III & & & & & & & & \\
\hline 45410 & 6 Lyn & K0III-IV & & & & & & & & \\
\hline 124897 & $\alpha$ Boo & K1III & & & & & & & & \\
\hline 3651 & $54 \mathrm{Psc}$ & $\mathrm{K} 0 \mathrm{~V}$ & & & & & & & & \\
\hline 32147 & HR 1614 & K5V & & & & & & & & \\
\hline
\end{tabular}

camera Astromed-3200 and a $1152 \times 770$ pixel EEV P88200 CCD detector. The wavelength range covers from 3765 to $9865 \AA$, for the first run, and from 3632 to $10800 \AA$, for the second run. The reciprocal dispersion ranges from 0.07 to $0.18 \AA /$ pixel and the spectral resolution, determined as the full width at half maximum (FWHM) of the arc comparison lines, ranges from 0.15 to $0.60 \AA$, in both runs.

We also analyse echelle spectra obtained during a 10night run in 12-21 January 1998 (McD98 hereafter), using the $2.1 \mathrm{~m}$ Otto Struve telescope at McDonald Observatory (Texas, U.S.A.) and the Sandiford Cassegrain Echelle Spectrograph (McCarthy et al. 1993). This instrument is a prism cross-dispersed echelle mounted at the Cassegrain focus and it is used with a $1200 \times 400$ Reticon CCD detector. The spectrograph setup was chosen to cover the H $\alpha(6563 \AA)$ and Ca II IRT $(8498,8542,8662 \AA)$ lines. The wavelength coverage is about $6400-8800 \AA$ and the reciprocal dispersion ranges from 0.06 to $0.08 \AA /$ pixel. The spectral resolution, determined as the FWHM of the arc comparison lines, ranges from 0.13 to $0.20 \AA$. In one of the nights, we changed the spectrograph setup to include the He I $\mathrm{D}_{3}(5876 \AA)$ and $\mathrm{Na}$ I $\mathrm{D}_{1}$ and $\mathrm{D}_{2}(5896,5890 \AA)$ lines, with wavelength coverage of $5600-7000 \AA$.

In addition, we dispose of echelle spectra taken in 7-8 January 1999 (INT99 hereafter), with the $2.5 \mathrm{~m}$ Isaac Newton Telescope (INT) at the Observatorio del Roque de Los Muchachos (La Palma, Spain) using the ESA-MUSICOS spectrograph. This is a fibre-fed cross-dispersed echelle spectrograph, built at the ESA Space Science Department in ESTEC as a replica of the first MUSICOS spectrograph built at Meudon-Paris Observatory (Baudrand \& Böhm 1992) and devel- oped as part of MUlti-SIte COntinuous Spectroscopy $\left(\right.$ MUSICOS $\left.^{1}\right)$ project. During this observing run, a $2148 \times 2148$ pixel SITe1 CCD detector was used obtaining wavelength coverage from $3950 \AA$ to $9890 \AA$. The reciprocal dispersion ranges from 0.06 to $0.12 \AA$ and the spectral resolution (FWHM of the of the arc comparison lines) from 0.15 to $0.4 \AA$.

In Table 1 we give the observing log. For each observation we list date, UT, orbital phase $(\varphi)$ and the signal to noise ratio $(S / N)$ obtained in the $\mathrm{H} \alpha$ line region. Table 2 shows the HD number, name and stellar parameters of the active binary systems and the non active stars used as reference stars in the spectral subtraction. The $B-V$ and $V-R$ color indexes and the radius are obtained from the relation with spectral type given by Landolt-Börnstein (Schmidt-Kaler 1982) when individual values are not given in the literature. Other parameters are given by CABS Catalog (Strassmeier et al. 1993) or taken from the references given in the individual results of each star.

The spectra have been extracted using the standard reduction procedures in the $\mathrm{IRAF}^{2}$ package (bias subtraction, flat-field division and optimal extraction of the spectra). The wavelength calibration was obtained by taking spectra of a Th-Ar lamp, for NOT and McDonald runs, and a $\mathrm{Cu}-\mathrm{Ar}$ lamp, for INT run. Finally, the spectra have been normalized by a polynomial fit to the observed continuum.

\footnotetext{
1 http://www.ucm.es/info/Astrof/MUSICOS.html

2 IRAF is distributed by the National Optical Observatory, which is operated by the Association of Universities for Research in Astronomy, Inc., under contract with the National Science Foundation.
} 
Table 3. $\mathrm{H} \alpha$ line measures in the observed and subtracted spectra

\begin{tabular}{|c|c|c|c|c|c|c|c|c|c|c|c|c|}
\hline \multirow[b]{2}{*}{ Name } & \multirow[b]{2}{*}{ Obs. } & \multirow[b]{2}{*}{$\varphi$} & \multirow[b]{2}{*}{$\mathrm{E}$} & \multirow[b]{2}{*}{$S_{\mathrm{H}} / S_{\mathrm{C}}$} & \multicolumn{4}{|c|}{ Observed $\mathrm{H} \alpha$ Spectrum } & \multicolumn{4}{|c|}{ Subtracted $\mathrm{H} \alpha$ Spectrum } \\
\hline & & & & & $\mathrm{H} \alpha$ & $\begin{array}{l}W_{\text {obs }} \\
(\AA)\end{array}$ & $R_{\mathrm{c}}$ & $\begin{array}{c}E W \\
(\AA)\end{array}$ & $\begin{array}{c}W_{\text {sub }} \\
(\AA)\end{array}$ & $I$ & $\begin{array}{c}E W^{*} \\
(\AA)\end{array}$ & $\log F_{\mathrm{S}}$ \\
\hline UX Ari & NOT 96 & 0.96 & $\mathrm{C}$ & $0.30 / 0.70$ & $\mathrm{E}$ & 1.44 & 1.27 & 0.42 & 2.28 & 0.65 & 2.13 & 6.89 \\
\hline $12 \mathrm{Cam}$ & NOT 98 & 0.64 & - & - & $\mathrm{A}$ & 2.04 & 0.47 & 1.16 & 1.02 & 0.28 & 0.34 & 5.99 \\
\hline V1149 Ori & NOT 98 & 0.89 & - & - & $\mathrm{A}$ & 2.35 & 0.62 & 0.93 & 1.10 & 0.47 & 0.58 & 6.04 \\
\hline OU Gem & NOT 96 & 0.80 & $\mathrm{H}$ & 0.70 & $\mathrm{~A}$ & 1.17 & 0.61 & 0.49 & 1.41 & 0.27 & 0.61 & 6.29 \\
\hline$"$ & $"$ & $"$ & $\mathrm{C}$ & 0.30 & $\mathrm{~A}$ & 1.16 & 0.93 & 0.08 & 1.77 & 0.17 & 1.03 & 6.31 \\
\hline$"$ & NOT 98 & 0.19 & $\mathrm{H}$ & 0.70 & A & 1.23 & 0.60 & 0.53 & 1.28 & 0.24 & 0.51 & 6.21 \\
\hline$"$ & $"$ & $"$ & $\mathrm{C}$ & 0.30 & $\mathrm{~A}$ & 0.89 & 0.91 & 0.07 & 1.32 & 0.14 & 0.70 & 6.14 \\
\hline$"$ & NOT 98 & 0.77 & $\mathrm{H}$ & 0.70 & A & 1.34 & 0.60 & 0.56 & 1.12 & 0.25 & 0.46 & 6.17 \\
\hline$"$ & $"$ & $"$ & $\mathrm{C}$ & 0.30 & $\mathrm{~A}$ & 0.60 & 0.96 & 0.02 & 1.40 & 0.21 & 0.93 & 6.26 \\
\hline$"$ & INT 99 & 0.70 & $\mathrm{H}$ & 0.70 & A & 1.26 & 0.67 & 0.46 & 1.35 & 0.29 & 0.67 & 6.33 \\
\hline$"$ & $"$ & $"$ & $\mathrm{C}$ & 0.30 & A & 0.60 & 0.92 & 0.05 & 1.48 & 0.10 & 0.50 & 5.99 \\
\hline$\sigma$ Gem & NOT 96 & 0.81 & - & - & $\mathrm{A}$ & 1.85 & 0.37 & 1.24 & 0.96 & 0.17 & 0.18 & 5.50 \\
\hline$"$ & NOT 98 & 0.88 & - & - & $\mathrm{A}$ & 1.69 & 0.49 & 0.95 & 1.13 & 0.27 & 0.34 & 5.77 \\
\hline BF Lyn & NOT 98 & 0.02 & $\mathrm{~T}$ & $0.50 / 0.50$ & $\mathrm{~F}$ & - & 0.87 & - & 1.53 & 0.62 & 0.99 & 6.60 \\
\hline$"$ & INT 99 & 0.11 & $\mathrm{H}$ & 0.50 & A & 0.94 & 0.82 & 0.18 & 1.32 & 0.32 & 0.91 & 6.56 \\
\hline$"$ & $"$ & $"$ & $\mathrm{C}$ & 0.50 & $\mathrm{~F}$ & - & 0.94 & - & 1.45 & 0.30 & 0.87 & 6.54 \\
\hline$"$ & McD 98 & 0.12 & $\mathrm{H}$ & $"$ & $\mathrm{~A}$ & 1.06 & 0.80 & 0.20 & 1.25 & 0.29 & 0.80 & 6.51 \\
\hline$"$ & $"$ & $"$ & $\mathrm{C}$ & $"$ & A & 1.07 & 0.85 & 0.16 & 1.21 & 0.23 & 0.57 & 6.36 \\
\hline$"$ & McD 98 & 0.16 & $\mathrm{H}$ & $"$ & A & 1.26 & 0.81 & 0.24 & 1.21 & 0.27 & 0.77 & 6.49 \\
\hline$"$ & $"$ & $"$ & $\mathrm{C}$ & $"$ & $\mathrm{~A}$ & 0.94 & 0.80 & 0.17 & 1.21 & 0.20 & 0.52 & 6.32 \\
\hline$"$ & McD 98 & 0.18 & $\mathrm{H}$ & $"$ & $\mathrm{~A}$ & 1.31 & 0.82 & 0.24 & 1.23 & 0.27 & 0.77 & 6.49 \\
\hline$"$ & $"$ & $"$ & $\mathrm{C}$ & $"$ & A & 0.99 & 0.83 & 0.16 & 1.22 & 0.21 & 0.55 & 6.34 \\
\hline$"$ & McD 98 & 0.40 & $\mathrm{H}$ & $"$ & $\mathrm{~A}$ & 1.16 & 0.84 & 0.20 & 1.41 & 0.28 & 0.87 & 6.54 \\
\hline$"$ & $"$ & $"$ & $\mathrm{C}$ & $"$ & $\mathrm{~A}$ & 0.94 & 0.87 & 0.12 & 1.29 & 0.25 & 0.59 & 6.37 \\
\hline$"$ & NOT 96 & 0.43 & $\mathrm{~T}$ & $"$ & $\mathrm{~F}$ & - & 0.88 & - & 2.06 & 0.50 & 0.99 & 6.60 \\
\hline$"$ & NOT 98 & 0.49 & $\mathrm{~T}$ & $"$ & $\mathrm{~F}$ & - & 0.78 & - & 1.38 & 0.60 & 0.96 & 6.58 \\
\hline$"$ & NOT 98 & 0.54 & $\mathrm{~T}$ & $"$ & $\mathrm{~F}$ & - & 0.84 & - & 1.84 & 0.54 & 0.98 & 6.59 \\
\hline$"$ & $\mathrm{McD} 98$ & 0.67 & $\mathrm{H}$ & $"$ & $\mathrm{~A}$ & 1.03 & 0.78 & 0.23 & 1.29 & 0.26 & 0.76 & 6.48 \\
\hline$"$ & $"$ & $"$ & $\mathrm{C}$ & $"$ & $\mathrm{~A}$ & 1.15 & 0.89 & 0.14 & 1.28 & 0.22 & 0.67 & 6.43 \\
\hline$"$ & McD 98 & 0.92 & $\mathrm{H}$ & $"$ & $\mathrm{~A}$ & 1.04 & 0.81 & 0.22 & 1.12 & 0.29 & 0.67 & 6.43 \\
\hline$"$ & $"$ & $"$ & $\mathrm{C}$ & $"$ & $\mathrm{~A}$ & 1.15 & 0.89 & 0.14 & 1.21 & 0.26 & 0.70 & 6.45 \\
\hline IL Hya & NOT 98 & 0.02 & $\mathrm{C}$ & $0.03 / 0.97$ & $\mathrm{~A}$ & 1.63 & 0.63 & 0.63 & 1.32 & 0.41 & 0.63 & 6.23 \\
\hline FG UMa & NOT 98 & - & - & - & $\mathrm{A}$ & 1.37 & 0.73 & 0.39 & 1.29 & 0.51 & 0.81 & 6.56 \\
\hline$"$ & NOT 98 & - & - & - & $\mathrm{A}$ & 1.05 & 0.80 & 0.22 & 1.85 & 0.60 & 1.03 & 6.66 \\
\hline LR Hya & NOT 98 & 0.58 & $\mathrm{H}$ & 0.50 & $\mathrm{~A}$ & 1.45 & 0.61 & 0.59 & 1.36 & 0.07 & 0.14 & 5.87 \\
\hline$"$ & $"$ & $"$ & $\mathrm{C}$ & 0.50 & A & 1.73 & 0.67 & 0.60 & 1.04 & 0.09 & 0.22 & 6.07 \\
\hline HU Vir & NOT 98 & 0.29 & - & - & $\mathrm{E}$ & 2.59 & 1.43 & 1.34 & 2.07 & 1.12 & 2.47 & 6.71 \\
\hline$"$ & NOT 98 & 0.38 & - & - & $\mathrm{E}$ & 2.21 & 1.31 & 0.71 & 1.80 & 0.96 & 1.91 & 6.60 \\
\hline$"$ & McD 98 & 0.44 & - & - & $\mathrm{E}$ & 2.81 & 1.23 & 0.63 & 2.09 & 0.85 & 1.95 & 6.61 \\
\hline$"$ & McD 98 & 0.54 & - & - & $\mathrm{E}$ & 2.10 & 1.25 & 0.54 & 2.02 & 0.79 & 1.66 & 6.54 \\
\hline$"$ & McD 98 & 0.64 & - & - & $\mathrm{E}$ & 2.93 & 1.22 & 0.59 & 2.18 & 0.81 & 1.82 & 6.58 \\
\hline$"$ & McD 98 & 0.73 & - & - & $\mathrm{E}$ & 3.57 & 1.09 & 0.25 & 1.90 & 0.68 & 1.41 & 6.47 \\
\hline$"$ & McD 98 & 0.83 & - & - & $\mathrm{E}$ & 3.28 & 1.11 & 0.26 & 1.99 & 0.68 & 1.41 & 6.47 \\
\hline$"$ & McD 98 & 0.93 & - & - & $\mathrm{E}$ & 1.19 & 1.13 & 0.15 & 1.91 & 0.65 & 1.31 & 6.44 \\
\hline$"$ & $\mathrm{McD} 98$ & 0.02 & - & - & $\mathrm{E}$ & 1.70 & 1.20 & 0.30 & 2.01 & 0.71 & 1.58 & 6.52 \\
\hline$"$ & McD 98 & 0.12 & - & - & $\mathrm{E}$ & 2.37 & 1.28 & 0.61 & 2.21 & 0.80 & 1.89 & 6.60 \\
\hline DK Dra & NOT 96 & 0.90 & $\mathrm{~T}$ & $0.50 / 0.50$ & $\mathrm{~A}$ & 2.07 & 0.64 & 0.74 & 1.87 & 0.32 & 0.57 & 6.16 \\
\hline$"$ & NOT 98 & 0.84 & $\mathrm{H}$ & 0.50 & $\mathrm{~A}$ & 1.88 & 0.68 & 0.64 & 1.24 & 0.18 & 0.43 & 6.04 \\
\hline$"$ & $"$ & $"$ & $\mathrm{C}$ & 0.50 & $\mathrm{~A}$ & 1.17 & 0.81 & 0.21 & 1.23 & 0.25 & 0.66 & 6.22 \\
\hline BQ CVn & NOT 98 & - & $\mathrm{C}$ & $0.20 / 0.80$ & $\mathrm{~A}$ & 1.94 & 0.58 & 0.77 & 1.39 & 0.34 & 0.61 & 6.25 \\
\hline IS Vir & NOT 98 & - & - & - & $\mathrm{A}$ & 1.50 & 0.65 & 0.58 & 1.24 & 0.47 & 0.70 & 6.07 \\
\hline BL CVn & NOT 98 & 0.21 & $\mathrm{C}$ & $0.07 / 0.93$ & $\mathrm{~A}$ & 2.10 & 0.55 & 0.98 & 1.03 & 0.24 & 0.30 & 5.94 \\
\hline BM CVn & NOT 98 & 0.26 & - & - & $\mathrm{A}$ & 1.55 & 0.76 & 0.35 & 1.30 & 0.60 & 0.89 & 6.35 \\
\hline MS Ser & CAHA95 & 0.93 & $\mathrm{P}$ & $0.15 / 0.85$ & $\mathrm{~F}$ & - & 0.95 & - & 1.22 & 0.63 & 1.01 & 6.44 \\
\hline$"$ & NOT 98 & 0.21 & $\mathrm{P}$ & " & $\mathrm{A}$ & 0.77 & 0.90 & 0.08 & 1.33 & 0.55 & 1.02 & 6.44 \\
\hline$"$ & NOT 98 & 0.54 & $\mathrm{P}$ & $"$ & $\mathrm{~A}$ & 1.00 & 0.85 & 0.16 & 1.52 & 0.57 & 1.13 & 6.48 \\
\hline
\end{tabular}

* $E W$ corrected for the contribution of each component to the total continuum.

\section{Spectroscopic features analysed}

\subsection{Chromospheric activity indicators}

The echelle spectra analysed in this paper allow us to study the behaviour of the different optical chromospheric activity indicators formed at different atmospheric heights: $\mathrm{Na} \mathrm{I}_{1}, \mathrm{D}_{2}$ and $\mathrm{Mg}$ I b triplet (upper photosphere and lower chromosphere), Ca II IRT lines (lower chromosphere), $\mathrm{H} \alpha, \mathrm{H} \beta$, Ca II $\mathrm{H} \& \mathrm{~K}$ (middle chromosphere) and He I $\mathrm{D}_{3}$ (upper chromosphere). The chromospheric contribution in these features has been determined using the spectral subtraction technique described in detail by Montes et al. (1995a,b,c), (see Papers I and II). The synthesized spectrum was constructed using the program STARMOD developed at Penn State (Barden 1985). The inactive stars used as 
Table 4. Parameters of the broad and narrow Gaussian components used in the fit of the $\mathrm{H} \alpha$ subtracted spectra

\begin{tabular}{|c|c|c|c|c|c|c|c|c|c|c|}
\hline \multirow[b]{2}{*}{ Name } & \multirow[b]{2}{*}{ Obs. } & \multirow[b]{2}{*}{$\varphi$} & \multicolumn{4}{|c|}{$\mathrm{H} \alpha$ broad component } & \multicolumn{4}{|c|}{$\mathrm{H} \alpha$ narrow component } \\
\hline & & & $I$ & $\begin{array}{c}F W H M \\
(\AA) \\
\end{array}$ & $\begin{array}{c}E W_{\mathrm{B}} \\
(\AA)\end{array}$ & $\begin{array}{c}E W_{\mathrm{B}} / E W_{\mathrm{T}} \\
(\%)\end{array}$ & $\bar{I}$ & $\begin{array}{c}F W H M \\
(\AA) \\
\end{array}$ & $\begin{array}{c}E W_{\mathrm{N}} \\
(\AA)\end{array}$ & $\begin{array}{c}E W_{\mathrm{N}} / E W_{\mathrm{T}} \\
(\%)\end{array}$ \\
\hline HU Vir & NOT 98 & 0.29 & 0.302 & 3.643 & 1.172 & 47.5 & 0.787 & 1.545 & 1.294 & 52.5 \\
\hline$"$ & NOT 98 & 0.38 & 0.431 & 2.434 & 1.117 & 58.8 & 0.566 & 1.297 & 0.782 & 41.2 \\
\hline$"$ & McD 98 & 0.44 & 0.275 & 3.848 & 1.128 & 57.9 & 0.589 & 1.303 & 0.818 & 42.1 \\
\hline$"$ & $\operatorname{McD} 98$ & 0.54 & 0.223 & 2.296 & 0.544 & 32.2 & 0.724 & 1.486 & 1.145 & 67.8 \\
\hline$"$ & McD 98 & 0.64 & 0.351 & 3.283 & 1.226 & 66.3 & 0.464 & 1.257 & 0.621 & 33.7 \\
\hline$"$ & McD 98 & 0.73 & 0.267 & 2.993 & 0.849 & 59.9 & 0.436 & 1.223 & 0.568 & 40.1 \\
\hline$"$ & McD 98 & 0.83 & 0.231 & 3.085 & 0.759 & 53.3 & 0.459 & 1.359 & 0.664 & 46.7 \\
\hline$"$ & McD 98 & 0.93 & 0.223 & 3.017 & 0.717 & 54.1 & 0.437 & 1.304 & 0.606 & 45.9 \\
\hline$"$ & McD 98 & 0.02 & 0.191 & 3.675 & 0.748 & 47.1 & 0.536 & 1.469 & 0.839 & 52.9 \\
\hline$"$ & McD 98 & 0.12 & 0.271 & 3.760 & 1.085 & 56.3 & 0.552 & 1.434 & 0.844 & 43.7 \\
\hline
\end{tabular}

Table 5. $\mathrm{H} \beta$ line measurements in the observed and subtracted spectra

\begin{tabular}{|c|c|c|c|c|c|c|c|c|c|c|c|}
\hline \multirow[b]{2}{*}{ Name } & \multirow[b]{2}{*}{ Obs. } & \multirow[b]{2}{*}{$\varphi$} & \multirow[b]{2}{*}{$\mathrm{E}$} & \multicolumn{3}{|c|}{ Observed $\mathrm{H} \beta$ Spectrum } & \multicolumn{3}{|c|}{ Subtracted $\mathrm{H} \beta$ Spectrum } & \multirow[b]{2}{*}{$\frac{E W(\mathrm{H} \alpha)}{E W(\mathrm{H} \beta)}$} & \multirow[b]{2}{*}{$\frac{E_{\mathrm{H} \alpha}}{E_{\mathrm{H} \beta}}$} \\
\hline & & & & $\begin{array}{c}W_{\text {obs }} \\
(\AA)\end{array}$ & $R_{\mathrm{c}}$ & $\begin{array}{l}E W \\
(\AA)\end{array}$ & $\begin{array}{c}W_{\text {sub }} \\
(\AA)\end{array}$ & $I$ & $\begin{array}{c}E W^{*} \\
(\AA)\end{array}$ & & \\
\hline UX Ari & NOT 96 & 0.95 & $\mathrm{C}$ & 1.02 & 0.57 & 0.48 & 1.23 & 0.29 & 0.54 & 3.94 & 4.24 \\
\hline $12 \mathrm{Cam}$ & NOT 98 & 0.64 & - & 1.39 & 0.24 & 1.15 & 0.42 & 0.12 & 0.06 & 5.67 & 7.90 \\
\hline V1149 Ori & NOT 98 & 0.89 & - & 1.70 & 0.31 & 1.24 & 0.51 & 0.21 & 0.13 & 4.46 & 7.14 \\
\hline OU Gem & NOT 96 & 0.80 & $\mathrm{H}$ & 1.09 & 0.48 & 0.62 & 0.73 & 0.16 & 0.20 & 3.05 & 3.84 \\
\hline$"$ & $"$ & $"$ & $\mathrm{C}$ & 0.45 & 0.74 & 0.12 & 0.33 & 0.18 & 0.20 & 5.15 & 9.03 \\
\hline$"$ & NOT 98 & 0.19 & $\mathrm{H}$ & 1.03 & 0.42 & 0.66 & 0.62 & 0.12 & 0.13 & 3.92 & 4.93 \\
\hline$"$ & $"$ & $"$ & $\mathrm{C}$ & 0.44 & 0.90 & 0.05 & 0.93 & 0.10 & 0.30 & 2.33 & 4.09 \\
\hline$"$ & NOT 98 & 0.77 & $\mathrm{H}$ & 1.07 & 0.47 & 0.61 & 0.78 & 0.13 & 0.16 & 2.87 & 3.62 \\
\hline$"$ & $"$ & $"$ & $\mathrm{C}$ & 0.36 & 0.80 & 0.08 & 0.57 & 0.16 & 0.33 & 2.82 & 4.95 \\
\hline$"$ & INT 99 & 0.70 & $\mathrm{H}$ & 0.89 & 0.64 & 0.34 & 0.75 & 0.12 & 0.14 & 4.79 & 6.03 \\
\hline$"$ & $"$ & $"$ & $\mathrm{C}$ & 0.34 & 0.90 & 0.04 & 0.23 & 0.06 & 0.10 & 5.00 & 8.78 \\
\hline$\sigma$ Gem & NOT 96 & 0.81 & - & 1.27 & 0.24 & 1.03 & 0.63 & 0.07 & 0.04 & 4.50 & 7.20 \\
\hline$"$ & NOT 98 & 0.88 & - & 1.33 & 0.28 & 1.00 & 0.59 & 0.10 & 0.07 & 4.86 & 7.78 \\
\hline BF Lyn & NOT 98 & 0.02 & $\mathrm{~T}$ & - & 0.62 & - & 1.23 & 0.31 & 0.36 & 2.75 & 3.31 \\
\hline$"$ & INT 99 & 0.11 & $\mathrm{H}$ & - & 0.40 & - & 1.16 & 0.13 & 0.36 & 2.53 & 3.04 \\
\hline$"$ & $"$ & $"$ & $\mathrm{C}$ & - & 0.89 & - & 0.76 & 0.22 & 0.35 & 2.48 & 2.98 \\
\hline$"$ & NOT 96 & 0.43 & $\mathrm{~T}$ & - & 0.69 & - & 1.71 & 0.31 & 0.57 & 1.74 & 2.09 \\
\hline$"$ & NOT 98 & 0.49 & $\mathrm{~T}$ & - & 0.56 & - & 0.77 & 0.39 & 0.35 & 2.74 & 3.29 \\
\hline$"$ & NOT 98 & 0.54 & $\mathrm{~T}$ & - & 0.61 & - & 1.05 & 0.40 & 0.39 & 2.51 & 3.02 \\
\hline IL Hya & NOT 98 & 0.02 & $\mathrm{C}$ & 1.27 & 0.35 & 0.87 & 0.68 & 0.17 & 0.13 & 4.84 & 6.26 \\
\hline FG UMa & NOT 98 & - & - & 1.42 & 0.35 & 0.98 & 0.39 & 0.15 & 0.10 & 8.10 & 9.00 \\
\hline$"$ & NOT 98 & - & - & 1.28 & 0.38 & 0.85 & 0.59 & 0.19 & 0.14 & 7.36 & 8.17 \\
\hline LR Hya & NOT 98 & 0.58 & $\mathrm{H}$ & 1.42 & 0.56 & 0.63 & - & 0.00 & 0.00 & - & - \\
\hline & $"$ & $"$ & $\mathrm{C}$ & 1.25 & 0.59 & 0.57 & - & 0.00 & 0.00 & - & - \\
\hline HU Vir & NOT 98 & 0.29 & - & 1.27 & 0.73 & 0.32 & 1.19 & 0.49 & 0.60 & 4.12 & 5.58 \\
\hline$"$ & NOT 98 & 0.38 & - & 1.57 & 0.77 & 0.37 & 1.08 & 0.46 & 0.52 & 3.67 & 4.95 \\
\hline DK Dra & NOT 96 & 0.90 & $\mathrm{~T}$ & 1.27 & 0.45 & 0.69 & 1.36 & 0.16 & 0.25 & 2.29 & 3.31 \\
\hline$"$ & NOT 98 & 0.84 & $\mathrm{H}$ & 1.69 & 0.44 & 0.85 & - & 0.00 & 0.00 & - & - \\
\hline$"$ & $"$ & $"$ & $\mathrm{C}$ & 0.60 & 0.60 & 0.29 & 0.22 & 0.12 & 0.07 & 9.43 & 13.0 \\
\hline BQ CVn & NOT 98 & - & $\mathrm{C}$ & 1.32 & 0.40 & 0.83 & 0.96 & 0.14 & 0.18 & 3.89 & 4.55 \\
\hline IS Vir & NOT 98 & - & - & 1.27 & 0.31 & 0.97 & 0.60 & 0.20 & 0.13 & 5.38 & 8.93 \\
\hline BL CVn & NOT 98 & 0.21 & $\mathrm{C}$ & 1.49 & 0.34 & 1.04 & - & 0.00 & 0.00 & - & - \\
\hline BM CVn & NOT 98 & 0.26 & - & 1.28 & 0.39 & 0.85 & 0.67 & 0.27 & 0.20 & 4.45 & 6.67 \\
\hline MS Ser & CAHA95 & 0.93 & $\mathrm{P}$ & 1.45 & 0.55 & 0.59 & 0.95 & 0.29 & 0.33 & 3.06 & 4.04 \\
\hline$"$ & NOT 98 & 0.21 & $\mathrm{P}$ & 1.22 & 0.60 & 0.50 & 0.80 & 0.37 & 0.40 & 2.55 & 3.36 \\
\hline$"$ & NOT 98 & 0.54 & $\mathrm{P}$ & 1.42 & 0.50 & 0.72 & 0.79 & 0.29 & 0.29 & 3.90 & 5.14 \\
\hline
\end{tabular}

* $E W$ corrected for the contribution of each component to the total continuum.

reference stars in the spectral subtraction were observed during the same observing run as the active stars or were taken from our libraries of late-type stars (see Montes 1998). We have determined the excess emission equivalent width $(E W)$ (measured in the subtracted spectra) and converted to absolute chromospheric flux at the stellar surface. We have estimated the errors in the measured $E W$ taking into account the typical internal precisions of STARMOD $\left(0.5-2 \mathrm{~km} \mathrm{~s}^{-1}\right.$ in velocity shifts, $\pm 5 \mathrm{~km} \mathrm{~s}^{-1}$ in $V \sin i$, and $5 \%$ in intensity weights), the rms obtained in the fit between observed and synthesized spectra in the regions outside the chromospheric features (typically in the range $0.01-0.03$ ) and the standard deviations resulting in the $E W$ measurements. The estimated errors are in the range $10-20 \%$. For low active stars errors are larger and we have considered as a clear detection of excess emission or absorption in the chromospheric lines 
Table 6. Ca II H \& K and H $\epsilon$ lines measures in the observed and subtracted spectra

\begin{tabular}{|c|c|c|c|c|c|c|c|c|c|c|c|c|}
\hline \multirow[b]{2}{*}{ Name } & \multirow[b]{2}{*}{ Obs. } & \multirow[b]{2}{*}{$\varphi$} & \multirow[b]{2}{*}{$\mathrm{E}$} & \multicolumn{3}{|c|}{ Reconstruction } & \multicolumn{3}{|c|}{ Spectral subtraction } & \multicolumn{3}{|c|}{ Absolute flux } \\
\hline & & & & $\begin{array}{l}E W \\
(\mathrm{~K})\end{array}$ & $\begin{array}{l}E W \\
(\mathrm{H})\end{array}$ & $\begin{array}{l}E W \\
(\mathrm{H} \epsilon)\end{array}$ & $\begin{array}{c}E W^{*} \\
(\mathrm{~K})\end{array}$ & $\begin{array}{c}E W^{*} \\
(\mathrm{H})\end{array}$ & $\begin{array}{c}E W^{*} \\
(\mathrm{H} \epsilon)\end{array}$ & $\begin{array}{c}\log F \\
(\mathrm{~K})\end{array}$ & $\begin{array}{c}\log F \\
(\mathrm{H})\end{array}$ & $\begin{array}{c}\log F \\
(\mathrm{H} \epsilon)\end{array}$ \\
\hline UX Ari & NOT 96 & 0.95 & $\mathrm{C}$ & 1.61 & 1.34 & 0.12 & 2.76 & 2.68 & 0.44 & 6.92 & 6.90 & 6.12 \\
\hline $12 \mathrm{Cam}$ & NOT 98 & 0.64 & - & 1.52 & 1.24 & - & 1.98 & 1.75 & - & 6.45 & 6.40 & - \\
\hline V1149 Ori & NOT 98 & 0.89 & - & 1.96 & 1.51 & - & 2.45 & 2.33 & - & 6.15 & 6.12 & - \\
\hline OU Gem & NOT 96 & 0.80 & $\mathrm{H}$ & 0.48 & 0.35 & 0.07 & 0.77 & 0.54 & 0.10 & 6.02 & 5.87 & 5.13 \\
\hline$"$ & $"$ & $"$ & $\mathrm{C}$ & 0.31 & 0.19 & - & 1.07 & 0.70 & - & 5.68 & 5.50 & - \\
\hline$"$ & NOT 98 & 0.19 & $\mathrm{H}$ & 0.63 & 0.52 & - & 1.06 & 0.94 & - & 6.16 & 6.11 & - \\
\hline$"$ & $"$ & $"$ & $\mathrm{C}$ & 0.45 & 0.44 & 0.16 & 1.60 & 1.70 & 0.70 & 5.86 & 5.88 & 5.50 \\
\hline$"$ & NOT 98 & 0.77 & $\mathrm{H}$ & 0.65 & 0.66 & 0.07 & 1.08 & 1.08 & 0.31 & 6.17 & 6.17 & 5.63 \\
\hline$"$ & $"$ & $"$ & $\mathrm{C}$ & 0.49 & 0.38 & - & 1.83 & 1.43 & - & 5.92 & 5.81 & - \\
\hline$\sigma$ Gem & NOT 96 & 0.81 & - & 1.35 & 1.14 & 0.07 & 1.53 & 1.35 & - & 5.88 & 5.82 & - \\
\hline$"$ & NOT 98 & 0.88 & - & 1.32 & 1.20 & 0.02 & 1.83 & 1.87 & - & 5.96 & 5.97 & - \\
\hline BF Lyn & NOT 98 & 0.02 & $\mathrm{H}$ & 1.06 & 0.94 & 0.19 & 2.38 & 2.10 & 0.56 & 6.74 & 6.68 & 6.11 \\
\hline$"$ & $"$ & $"$ & $\mathrm{C}$ & 0.93 & 0.94 & 0.16 & 2.10 & 2.08 & 0.48 & 6.68 & 6.68 & 6.04 \\
\hline$"$ & NOT 96 & 0.43 & $\mathrm{H}$ & 1.12 & 0.96 & 0.13 & 2.64 & 2.52 & 0.84 & 6.78 & 6.76 & 6.29 \\
\hline$"$ & $"$ & $"$ & $\mathrm{C}$ & 1.04 & 0.89 & 0.25 & 2.48 & 2.30 & 0.96 & 6.76 & 6.72 & 6.34 \\
\hline$"$ & NOT 98 & 0.49 & $\mathrm{~T}$ & 1.89 & 1.75 & 0.30 & 3.96 & 3.72 & 0.88 & 6.96 & 6.93 & 6.31 \\
\hline$"$ & NOT 98 & 0.54 & $\mathrm{H}$ & 0.95 & 0.83 & 0.19 & 2.20 & 2.06 & 0.50 & 6.70 & 6.68 & 6.06 \\
\hline$"$ & $"$ & $"$ & $\mathrm{C}$ & 0.97 & 0.79 & 0.15 & 2.20 & 1.96 & 0.52 & 6.70 & 6.65 & 6.08 \\
\hline IL Hya & NOT 98 & 0.02 & $\mathrm{C}$ & 1.79 & 1.60 & - & 1.94 & 1.91 & - & 6.38 & 6.38 & - \\
\hline FG UMa & NOT 98 & - & - & 1.55 & 1.35 & - & 1.95 & 1.77 & - & 6.93 & 6.89 & - \\
\hline$"$ & NOT 98 & - & - & 1.68 & 1.35 & - & 2.05 & 1.82 & - & 6.96 & 6.90 & - \\
\hline LR Hya & NOT 98 & 0.58 & $\mathrm{H}$ & 0.29 & 0.27 & - & 1.06 & 1.04 & - & 6.67 & 6.66 & - \\
\hline$"$ & $"$ & $"$ & $\mathrm{C}$ & 0.29 & 0.24 & - & 1.06 & 0.88 & - & 6.67 & 6.59 & - \\
\hline HU Vir & NOT 98 & 0.29 & - & 2.67 & 2.68 & 0.52 & 2.75 & 2.96 & 0.62 & 6.44 & 6.48 & 5.80 \\
\hline$"$ & NOT 98 & 0.38 & - & 3.34 & 2.90 & 0.49 & 3.78 & 3.00 & 0.96 & 6.58 & 6.48 & 5.99 \\
\hline DK Dra & NOT 96 & 0.90 & $\mathrm{~T}$ & 0.79 & 0.37 & - & 1.41 & 1.19 & - & 6.18 & 6.11 & - \\
\hline$"$ & NOT 98 & 0.84 & $\mathrm{H}$ & 1.08 & 0.83 & - & 2.62 & 1.92 & - & 6.45 & 6.32 & - \\
\hline$"$ & $"$ & $"$ & $\mathrm{C}$ & 1.04 & 0.69 & - & 2.76 & 1.80 & - & 6.48 & 6.29 & - \\
\hline BQ CVn & NOT 98 & - & $\mathrm{C}$ & 1.30 & 1.14 & 0.04 & 1.69 & 1.65 & 0.16 & 6.39 & 6.38 & 5.36 \\
\hline IS Vir & NOT 98 & - & - & 1.07 & 1.05 & - & 2.10 & 2.05 & - & 5.99 & 5.97 & - \\
\hline BL CVn & NOT 98 & 0.21 & $\mathrm{C}$ & 1.28 & 1.21 & - & 1.85 & 1.77 & - & 6.43 & 6.41 & - \\
\hline $\mathrm{BM}$ CVn & NOT 98 & 0.26 & - & 3.08 & 2.45 & 0.17 & 3.25 & 2.98 & - & 6.55 & 6.51 & - \\
\hline MS Ser & NOT 98 & 0.21 & $\mathrm{P}$ & 2.05 & 1.83 & - & 2.59 & 2.74 & - & 6.70 & 6.66 & - \\
\hline$"$ & $"$ & $"$ & $\mathrm{~S}$ & 0.13 & 0.33 & - & 2.65 & 3.64 & - & 7.24 & 7.38 & - \\
\hline$"$ & NOT 98 & 0.54 & $\mathrm{~T}$ & 1.95 & 1.93 & 0.16 & 2.20 & 2.31 & 0.41 & 6.56 & 6.58 & 5.83 \\
\hline
\end{tabular}

* $E W$ corrected for the contribution of each component to the total continuum.

only when these features in the difference spectrum are larger than $3 \sigma$.

Table 3 gives the $\mathrm{H} \alpha$ line parameters, measured in the observed and subtracted spectra of the sample. The Col. 3 gives the orbital phase $(\varphi)$ for each spectrum. In the Col. 4 , $\mathrm{H}$ and $\mathrm{C}$ mean emission from the hot and cool components, respectively, and $T$ means that at these phases the spectral features cannot be deblended. The Col. 5 gives the relative contribution of the hot and cool components to the total continuum $\left(S_{\mathrm{H}}\right.$ and $\left.S_{\mathrm{C}}\right)$, respectively. The Col. 6 describes the observed $\mathrm{H} \alpha$ profile, i.e. whether the line is in absorption (A), in emission (E) or totally filled-in by emission $(\mathrm{F})$. The Cols. 7, 8 and 9 give the following parameters measured in the observed spectrum: the full width at half maximum $\left(W_{\text {obs }}\right)$, the residual intensity $\left(R_{\mathrm{c}}=\frac{F_{\mathrm{c}}}{F_{\text {cont }}}\right)$ and the equivalent width $(E W)$. The last four columns give the following parameters measured in the subtracted spectrum: the full width at half maximum $\left(W_{\text {sub }}\right)$, the peak emission intensity $(I)$, the excess $\mathrm{H} \alpha$ emission equivalent width $(E W(\mathrm{H} \alpha))$, corrected for the contribution of the components to the total continuum and the logarithm of the absolute flux at the stellar surface $\left(\log F_{\mathrm{S}}(\mathrm{H} \alpha)\right)$ obtained with the calibration of Hall (1996) as a function of $(V-R)$.

In Table 4 we list the parameters $(I, F W H M, E W)$ of the broad and narrow components used in the two Gaussian-component fit to the $\mathrm{H} \alpha$ subtracted emission profile. We have performed this fit in the stars that show broad wings. See the comments for each individual star in Sect. 4 and the interpretation of these components given in Sect. 5.

Table 5 gives the $\mathrm{H} \beta$ line parameters, measured in the observed and subtracted spectra, as in the case of the $\mathrm{H} \alpha$ line. In this table we also give the ratio of excess emission $E W$ in the $\mathrm{H} \alpha$ and $\mathrm{H} \beta$ lines, $\frac{E W(\mathrm{H} \alpha)}{E W(\mathrm{H} \beta)}$, and the ratio of excess emission $\frac{E_{\mathrm{H} \alpha}}{E_{\mathrm{H} \beta}}$ with the correction:

$\frac{E_{\mathrm{H} \alpha}}{E_{\mathrm{H} \beta}}=\frac{E W(\mathrm{H} \alpha)}{E W(\mathrm{H} \beta)} * 0.2444 * 2.512^{(B-R)}$

given by Hall \& Ramsey (1992) that takes into account the absolute flux density in these lines and the color difference in the components. We have used this ratio as a diagnostic for discriminating between the presence of plage-like and prominence-like material at the stellar surface, following the theoretical modelling by Buzasi (1989) who found that low $E_{\mathrm{H}} \alpha / E_{\mathrm{H}} \beta(\approx 1-2)$ can be achieved both in plages 
Table 7. Ca II IRT lines measures in the observed and subtracted spectra

\begin{tabular}{|c|c|c|c|c|c|c|c|c|c|c|c|c|c|}
\hline \multirow{3}{*}{ Name } & \multirow{3}{*}{ Obs. } & \multirow{3}{*}{$\varphi$} & \multirow{3}{*}{$\mathrm{E}$} & \multicolumn{3}{|c|}{ Reconstruction } & \multicolumn{3}{|c|}{ Spectral subtraction } & \multirow{3}{*}{$\frac{E W(\lambda 8542)}{E W(\lambda 8498)}$} & \multicolumn{3}{|c|}{ Absolute flux } \\
\hline & & & & $E W$ & $E W$ & $E W$ & $E W^{*}$ & $E W^{*}$ & $E W^{*}$ & & $\log F$ & $\log F$ & $\log F$ \\
\hline & & & & $\lambda 8498$ & $\lambda 8542$ & $\lambda 8662$ & $\lambda 8498$ & $\lambda 8542$ & $\lambda 8662$ & & $\lambda 8498$ & $\lambda 8542$ & $\lambda 8662$ \\
\hline UX Ari & NOT 96 & 0.95 & $\mathrm{C}$ & - & - & 0.31 & - & - & 1.33 & - & - & - & 6.63 \\
\hline $12 \mathrm{Cam}$ & NOT 98 & 0.64 & - & - & - & - & - & 0.64 & 0.47 & - & - & 6.28 & 6.14 \\
\hline V1149 Ori & NOT 98 & 0.89 & - & - & - & - & - & 0.83 & 0.71 & - & - & 6.27 & 6.21 \\
\hline OU Gem & NOT 96 & 0.80 & $\mathrm{H}$ & - & - & 0.02 & - & - & 0.56 & - & - & - & 6.16 \\
\hline$"$ & " & $"$ & $\mathrm{C}$ & - & - & 0.07 & - & - & 0.30 & - & - & - & 5.76 \\
\hline$"$ & NOT 98 & 0.19 & $\mathrm{H}$ & - & 0.06 & - & - & 0.53 & 0.40 & - & - & 6.14 & 6.02 \\
\hline$"$ & $"$ & $"$ & $\mathrm{C}$ & - & 0.14 & 0.16 & - & 0.37 & 0.20 & - & - & 5.85 & 5.59 \\
\hline$"$ & NOT 98 & 0.77 & $\mathrm{H}$ & - & 0.05 & - & - & 0.58 & 0.50 & - & - & 6.18 & 6.11 \\
\hline$"$ & " & $"$ & $\mathrm{C}$ & - & 0.13 & 0.10 & - & 0.63 & 0.27 & - & - & 6.09 & 5.72 \\
\hline$"$ & INT 99 & 0.70 & $\mathrm{H}$ & - & 0.02 & - & 0.58 & 0.96 & 1.01 & 1.66 & 6.18 & 6.40 & 6.42 \\
\hline$"$ & $"$ & $"$ & $\mathrm{C}$ & - & 0.06 & 0.07 & 0.27 & 0.53 & 0.50 & 1.96 & 5.72 & 6.02 & 5.99 \\
\hline$\sigma$ Gem & NOT 96 & 0.81 & - & - & - & - & - & - & 0.38 & - & - & - & 5.93 \\
\hline$"$ & NOT 98 & 0.88 & - & - & - & - & - & 0.84 & 0.59 & - & - & 6.26 & 6.11 \\
\hline BF Lyn & NOT 98 & 0.02 & $\mathrm{H}$ & - & 0.14 & 0.06 & - & 0.99 & 0.74 & - & - & 6.47 & 6.34 \\
\hline$"$ & $"$ & $"$ & $\mathrm{C}$ & - & 0.10 & 0.08 & - & 0.93 & 0.70 & - & - & 6.45 & 6.32 \\
\hline$"$ & INT 99 & 0.11 & $\mathrm{H}$ & 0.16 & 0.12 & 0.10 & 0.74 & 0.91 & 0.74 & 1.23 & 6.35 & 6.43 & 6.34 \\
\hline$"$ & $"$ & $"$ & $\mathrm{C}$ & 0.16 & 0.17 & 0.14 & 0.39 & 0.58 & 0.37 & 1.49 & 6.07 & 6.24 & 6.04 \\
\hline$"$ & McD 98 & 0.12 & $\mathrm{H}$ & - & 0.11 & 0.08 & 0.29 & 0.42 & 0.33 & 1.45 & 5.94 & 6.10 & 5.99 \\
\hline$"$ & " & $"$ & $\mathrm{C}$ & - & 0.11 & 0.08 & 0.52 & 0.49 & 0.66 & 0.94 & 6.19 & 6.17 & 6.30 \\
\hline$"$ & McD 98 & 0.16 & $\mathrm{H}$ & - & 0.12 & 0.09 & 0.27 & 0.34 & 0.33 & 1.26 & 5.91 & 6.01 & 5.99 \\
\hline$"$ & $"$ & $"$ & $\mathrm{C}$ & - & 0.18 & 0.10 & 0.45 & 0.45 & 0.66 & 1.00 & 6.13 & 6.13 & 6.30 \\
\hline$"$ & McD 98 & 0.18 & $\mathrm{H}$ & - & 0.17 & 0.08 & 0.28 & 0.38 & 0.31 & 1.36 & 5.92 & 6.06 & 5.97 \\
\hline$"$ & $"$ & $"$ & $\mathrm{C}$ & - & 0.16 & 0.07 & 0.55 & 0.68 & 0.77 & 1.24 & 6.21 & 6.31 & 6.36 \\
\hline$"$ & McD 98 & 0.40 & $\mathrm{H}$ & - & 0.10 & - & 0.32 & 0.43 & 0.57 & 1.34 & 5.98 & 6.11 & 6.23 \\
\hline$"$ & $"$ & $"$ & $\mathrm{C}$ & - & 0.09 & - & 0.57 & 0.44 & 0.62 & 0.77 & 6.23 & 6.12 & 6.27 \\
\hline$"$ & NOT 96 & 0.43 & $\mathrm{H}$ & - & - & 0.10 & - & - & 0.76 & - & - & - & 6.36 \\
\hline$"$ & $"$ & $"$ & $\mathrm{C}$ & - & - & 0.12 & - & - & 0.64 & - & - & - & 6.28 \\
\hline$"$ & NOT 98 & 0.49 & $\mathrm{~T}$ & - & 0.26 & 0.22 & - & 0.82 & 0.63 & - & - & 6.39 & 6.27 \\
\hline$"$ & NOT 98 & 0.54 & $\mathrm{H}$ & - & 0.14 & 0.09 & - & 0.83 & 0.72 & - & - & 6.39 & 6.33 \\
\hline$"$ & " & $"$ & $\mathrm{C}$ & - & 0.15 & 0.11 & - & 0.90 & 0.73 & - & - & 6.43 & 6.34 \\
\hline$"$ & McD 98 & 0.67 & $\mathrm{H}$ & - & 0.11 & 0.06 & 0.29 & 0.38 & 0.36 & 1.31 & 5.94 & 6.06 & 6.03 \\
\hline$"$ & $"$ & $"$ & $\mathrm{C}$ & - & 0.13 & 0.08 & 0.57 & 0.70 & 0.48 & 1.23 & 6.23 & 6.32 & 6.16 \\
\hline$"$ & McD 98 & 0.92 & $\mathrm{H}$ & - & 0.07 & 0.11 & 0.39 & 0.38 & 0.42 & 0.97 & 6.07 & 6.05 & 6.10 \\
\hline$"$ & " & $"$ & $\mathrm{C}$ & - & 0.12 & 0.13 & 0.53 & 0.62 & 0.58 & 1.17 & 6.20 & 6.27 & 6.24 \\
\hline IL Hya & NOT 98 & 0.02 & $\mathrm{C}$ & - & 0.15 & 0.05 & - & 1.01 & 0.79 & - & - & 6.44 & 6.34 \\
\hline FG UMa & NOT 98 & - & - & - & 0.04 & 0.01 & - & 0.84 & 0.55 & - & - & 6.48 & 6.29 \\
\hline & NOT 98 & - & - & - & - & - & - & 0.64 & 0.55 & - & - & 6.36 & 6.29 \\
\hline LR Hya & NOT 98 & 0.58 & $\mathrm{H}$ & - & - & - & - & 0.60 & 0.52 & - & - & 6.33 & 6.27 \\
\hline$"$ & $"$ & $"$ & $\mathrm{C}$ & - & - & - & - & 0.80 & 0.58 & - & - & 6.45 & 6.32 \\
\hline HU Vir & NOT 98 & 0.29 & - & - & 0.32 & 0.19 & - & 1.59 & 1.46 & - & - & 6.58 & 6.55 \\
\hline$"$ & NOT 98 & 0.38 & - & - & 0.25 & 0.14 & - & 1.19 & 1.04 & - & - & 6.46 & 6.40 \\
\hline$"$ & McD 98 & 0.44 & - & 0.32 & 0.30 & 0.22 & 0.83 & 1.41 & 0.65 & 1.70 & 6.30 & 6.53 & 6.19 \\
\hline$"$ & McD 98 & 0.64 & - & 0.24 & 0.13 & 0.20 & 0.66 & 1.10 & 0.88 & 1.67 & 6.20 & 6.42 & 6.32 \\
\hline$"$ & McD 98 & 0.73 & - & 0.38 & 0.17 & 0.32 & 0.64 & 0.93 & 0.71 & 1.45 & 6.19 & 6.35 & 6.23 \\
\hline$"$ & McD 98 & 0.83 & - & 0.15 & 0.12 & 0.12 & 0.62 & 0.89 & 0.81 & 1.43 & 6.17 & 6.33 & 6.29 \\
\hline$"$ & McD 98 & 0.93 & - & 0.09 & 0.18 & 0.14 & 0.63 & 0.89 & 0.57 & 1.41 & 6.18 & 6.33 & 6.14 \\
\hline$"$ & McD 98 & 0.02 & - & 0.19 & 0.27 & 0.18 & 0.63 & 1.13 & 0.60 & 1.79 & 6.18 & 6.43 & 6.16 \\
\hline$"$ & McD 98 & 0.12 & - & 0.35 & 0.18 & 0.09 & 0.71 & 1.10 & 0.47 & 1.55 & 6.23 & 6.42 & 6.05 \\
\hline DK Dra & NOT 96 & 0.90 & $\mathrm{~T}$ & - & - & - & - & - & 1.12 & - & - & - & 6.47 \\
\hline$"$ & NOT 98 & 0.84 & $\mathrm{H}$ & - & - & - & - & 0.65 & 0.47 & - & - & 6.24 & 6.09 \\
\hline$"$ & $"$ & $"$ & $\mathrm{C}$ & - & - & - & - & 1.59 & 0.98 & - & - & 6.63 & 6.41 \\
\hline BQ CVn & NOT 98 & - & $\mathrm{C}$ & - & 0.08 & 0.03 & - & 1.11 & 0.85 & - & - & 6.50 & 6.38 \\
\hline IS Vir & NOT 98 & - & - & - & - & - & - & 0.92 & 0.70 & - & - & 6.30 & 6.18 \\
\hline BL CVn & NOT 98 & 0.21 & $\mathrm{C}$ & - & - & - & - & 0.52 & 0.30 & - & - & 6.17 & 5.93 \\
\hline BM CVn & NOT 98 & 0.26 & - & - & 0.14 & 0.04 & - & 0.86 & 0.75 & - & - & 6.36 & 6.30 \\
\hline MS Ser & NOT 98 & 0.21 & $\mathrm{P}$ & - & 0.32 & 0.26 & - & 0.96 & 0.95 & - & - & 6.42 & 6.42 \\
\hline$"$ & $"$ & $"$ & $\mathrm{~S}$ & - & - & - & - & 0.47 & 0.53 & - & - & 6.11 & 6.16 \\
\hline$"$ & NOT 98 & 0.54 & $\mathrm{~T}$ & - & 0.30 & 0.21 & - & 1.05 & 0.75 & - & - & 6.46 & 6.31 \\
\hline
\end{tabular}

* $E W$ corrected for the contribution of each component to the total continuum.

and prominences viewed against the disk, but that high ratios $(\approx 3-15)$ can only be achieved in extended regions viewed off the limb. The study of chromospherically active binaries by Hall \& Ramsey (1992) has demonstrated the presence of large amounts of extended, prominence-like material in these stars.

We also analyse the possible filling-in of the core of the $\mathrm{Na}$ I $\mathrm{D}_{1}$ and $\mathrm{D}_{2}$ lines as other chromospheric activity indicator as well as the behaviour of the $\mathrm{He} \mathrm{I}_{3}$ line, which can be in absorption, filled-in due to frequent low-level flaring or in emission due to flares (see Papers I and II; Saar et al. 1997; Montes et al. 1996b, 1999).

Table 6 gives the $\mathrm{Ca}$ II $\mathrm{H} \& \mathrm{~K}$ and $\mathrm{H} \epsilon$ lines parameters, measured in the observed and subtracted spectra. In Cols. 5 and 6 we list the $E W$ for the $\mathrm{K}$ and $\mathrm{H}$ lines, obtained by reconstruction of the absorption line profile (described by Fernández-Figueroa et al. 1994, hereafter FFMCC). In Cols. 7, 8 and 9 we give the $E W$ for the $\mathrm{K}$, $\mathrm{H}$ and $\mathrm{H} \epsilon$ lines using the spectral subtraction technique (explained by Montes et al. 1995c, 1996a) and corrected 
for the contribution of the components to the total continuum. In Cols. 10, 11 and 12 we list the corresponding logarithm of the surface flux obtained by means of the linear relationship between the absolute surface flux at $3950 \AA$ (in erg $\mathrm{cm}^{-2} \mathrm{~s}^{-1} \AA^{-1}$ ) and the colour index $(V-R)$ by Pasquini et al. (1988).

Table 7 gives the Ca II IRT lines parameters, measured in the observed spectra by reconstruction of the absorption line profile and using the spectral subtraction. The columns of this table have the same meaning as in Table 6 for the Ca II H \& K lines. The absolute fluxes at the stellar surface have been obtained using the calibration of Hall (1996) as a function of $(V-R)$. For the observing runs in which the $\lambda 8542$ and $\lambda 8498$ lines are included we also give the ratio of excess emission $E W, E_{8542} / E_{8498}$, which is also an indicator of the type of chromospheric structure that produces the observed emission. In solar plages, values of $E_{8542} / E_{8498} \approx 1.5-3$ are measured, while in solar prominences the values are $\approx 9$, the limit of an optically thin emitting plasma (Chester 1991). However, the observations of active stars (Chester et al. 1994; Arévalo \& Lázaro 1999) indicate that these lines exhibit markedly different behaviour. The $E_{8542} / E_{8498}$ ratios found in these stars are smaller (closer to the optically thick value of one) than solar plages. These values indicate that the Ca II IRT emission arises predominantly in chromospheric plages.

\subsection{The $\mathrm{Li}$ I $\lambda 6707.8$ line}

The resonance doublet of $\mathrm{Li}$ I at $\lambda 6708 \AA$ is an important diagnostic of age in late-type stars since it is destroyed easily by thermonuclear reactions in the stellar interior. It is well-known that a large number of chromospherically active binaries shows Li I abundances higher than other stars of the same mass and evolutionary stage (Barrado et al. 1997, 1998; Paper I, Montes \& Ramsey 1998). This line is only included in our echelle spectra in the McD98 and INT99 observing runs. In Fig. 25 we have plotted representative spectra of OU Gem, BF Lyn and HU Vir in this spectral region. A K1III reference star with some photospheric lines identified has been also plotted in order to better identify the expected position of the $\mathrm{Li}$ I line. It was only possible to measure the equivalent width of the $\mathrm{Li}$ I absorption line in the SB1 system HU Vir. In the case of the SB2 systems OU Gem and BF Lyn the possible small absorption Li I of one or both components is blended with photospheric lines of the other component.

\section{Individual results}

In this section we describe the behaviour of the above mentioned chromospheric activity indicators for each star of the sample. The profiles of $\mathrm{H} \alpha, \mathrm{H} \beta$, Ca II $\mathrm{H} \& \mathrm{~K}$ and Ca II IRT are displayed from Figs. 1 to 23 . For each system we have plotted the observed spectrum (solid-line) and the synthesized spectrum (dashed-line) in the left panel and the subtracted spectrum (dotted line) in the right panel. The name of the star, the observing run (NOT96, NOT98, McD98, INT99) and the orbital phase $(\varphi)$ of each spectrum are given in every figure. The He $\mathrm{I}_{3}$ line, for selected stars of the sample, is displayed in Fig. 24.

\subsection{UX Ari (HD 21242)}

This double-lined spectroscopic binary (G5V/K0IV) is a well known RS CVn system and extensively studied in the literature (Carlos \& Popper 1971; Bopp \& Talcott 1978; Huenemoerder et al. 1989; Raveendran \& Mohin 1995). Recently, Duemmler \& Aarum (2000) have given a new orbit determination, which we have adopted in Table 2. Our previous $\mathrm{H} \alpha$ observations (Montes et al. 1995a,b) showed clear $\mathrm{H} \alpha$ emission above the continuum from the cool component. This emission was superimposed to the weak absorption of the hot component. Our spectrum in the Ca II H \& K region (Montes et al. 1995c) showed strong emission from the cool component and a weak $\mathrm{H} \epsilon$ emission line. We also detected a flare in this system through simultaneous $\mathrm{H} \alpha, \mathrm{Na}$ I $\mathrm{D}_{1}, \mathrm{D}_{2}$ and $\mathrm{He}$ I $\mathrm{D}_{3}$ observations (Montes et al. 1996b; Paper I).

In the new observation (NOT96) we observe intense emission in the $\mathrm{Ca}$ II $\mathrm{H} \& \mathrm{~K}, \mathrm{H} \epsilon, \mathrm{H} \alpha$ and $\mathrm{Ca}$ II $\lambda 8662$ lines and a filled-in absorption $\mathrm{H} \beta$ line from the cool component (see Fig. 1). The excess emission measured in all these activity indicators is larger than in our previous observations of this system in quiescent state in 1992 and 1995.

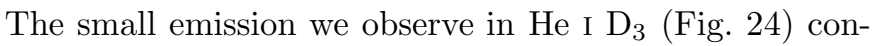
firms the high level of activity of UX Ari in this observation. The He $\mathrm{I}_{3}$ line has been observed as clear emission in this system in other occasions associated to flare-like events (Montes et al. 1996b and references therein).

\section{2. $12 \mathrm{Cam}$ (BM Cam, HD 32357)}

This single-lined spectroscopy binary was classified by Bidelman (1964) as a K0 giant. He also noticed Ca II H \& K emission. Later, Hall et al. (1995) revised the system spectroscopically and photometrically and obtained new values of orbital parameters, given in Table 2. Eker et al. (1995) observed that $\mathrm{H} \alpha$ profiles showed an asymmetric shape with a round shoulder in the red wing and a steeper blue wing. They also confirmed the variable $\mathrm{H} \alpha$ filling, which was suspected by Strassmeier et al. (1990) too. In our previous observations of this system (FFMCC) we found the $\mathrm{H} \alpha$ line filled-in and strong $\mathrm{Ca}$ II $\mathrm{H} \& \mathrm{~K}$ emission.

In the new spectrum (Fig. 2) we find strong emission in the $\mathrm{Ca}$ II $\mathrm{H} \& \mathrm{~K}$ lines. Thanks to the higher resolution of this spectrum it is possible to see now that 

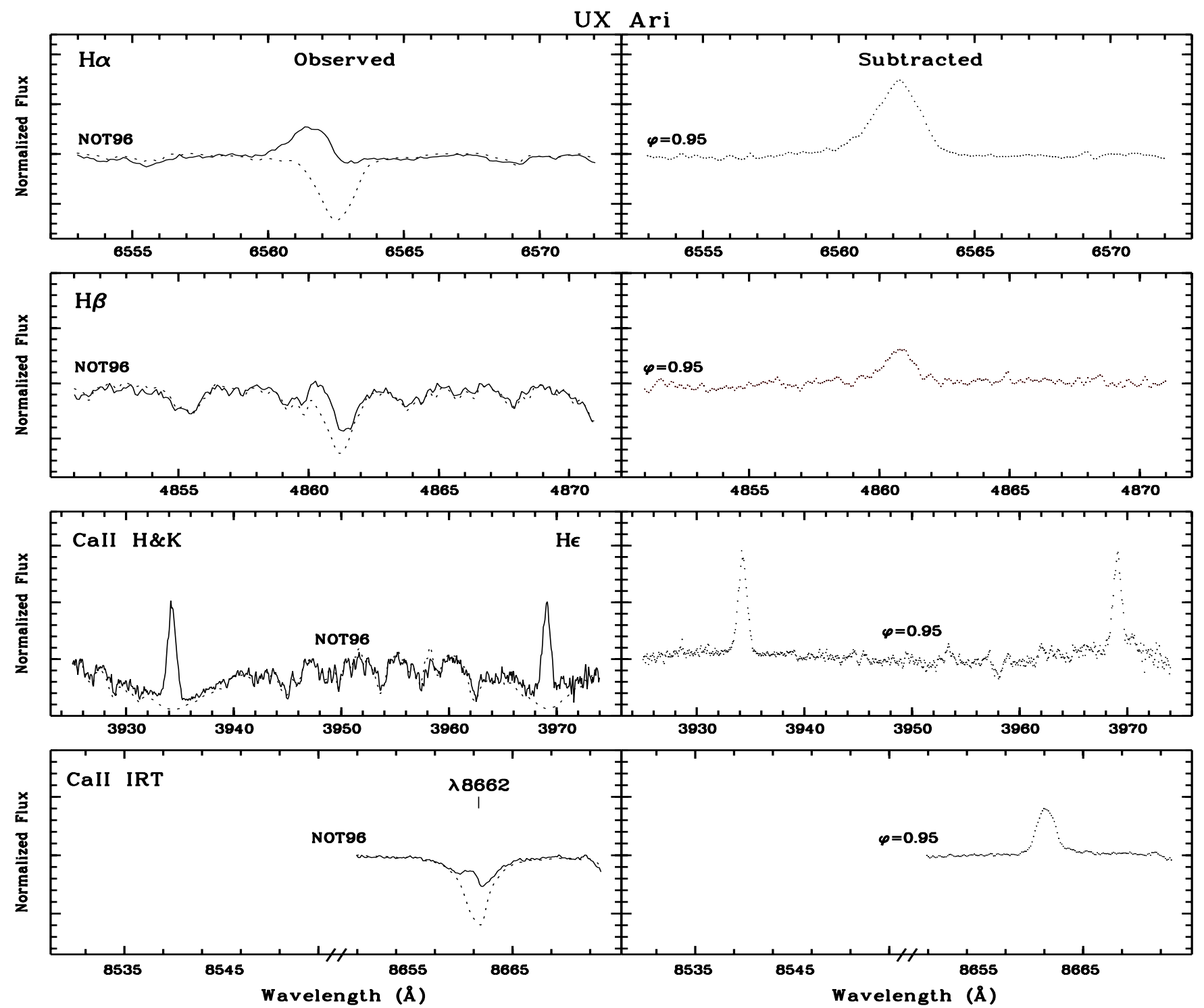

Fig. 1. $\mathrm{H} \alpha, \mathrm{H} \beta$, Ca II H \& K, and Ca II IRT spectra of UX Ari. Observed and synthetic spectra in the left panel and subtracted spectra in the right panel

both lines exhibit self-absorption with red asymmetry. Small $\mathrm{H} \epsilon$ emission is observed, but it was impossible to deblend it from the $\mathrm{Ca}$ II $\mathrm{H}$ line. Both $\mathrm{H} \alpha$ and $\mathrm{H} \beta$ appear in absorption with a slight filling-in, the $\mathrm{H} \alpha$ filling-in is smaller than the one corresponding to other epochs. The $\mathrm{H} \alpha$ line shows excess absorption in the red wing similar to the asymmetric shape of $\mathrm{H} \alpha$ observed by Eker et al. (1995). The application of the spectral subtraction technique reveals that the He I $\mathrm{D}_{3}$ line appears as an absorption feature (Fig. 24). This fact is more frequent in giants than in dwarfs (Paper I). Finally, the Ca II IRT $(\lambda \lambda$ 8542, $8662 \AA)$ absorption lines are clearly filled-in.

\subsection{V1149 Ori (HD 37824)}

This single-lined spectroscopic binary, classified as K1III + F by Bidelman \& MacConnell (1973), was listed as a G5IV star by Hirshfeld \& Sinnott (1982). Our previous observations revealed clear excess $\mathrm{H} \alpha$ emission (Montes et al. 1995a,b; Paper I), strong Ca II H \& K and $\mathrm{H} \epsilon$ emission (Montes et al. 1995c) and clear absorption in the He $\mathrm{I} \mathrm{D}_{3}$ line in the subtracted spectrum (Paper I).

In the new observation (NOT98, Fig. 3), the $\mathrm{H} \alpha$ and $\mathrm{H} \beta$ lines show a filled-in absorption profile, and clear absorption is observed in the blue wing of both lines. This excess absorption in the blue wing, not observed in our previous observations of this system, could be indicative of variable mass motion. To confirm this behaviour we took a new spectrum with the ESA-MUSICOS spectrograph in January 2000 (forthcoming paper) in which the blue wing 


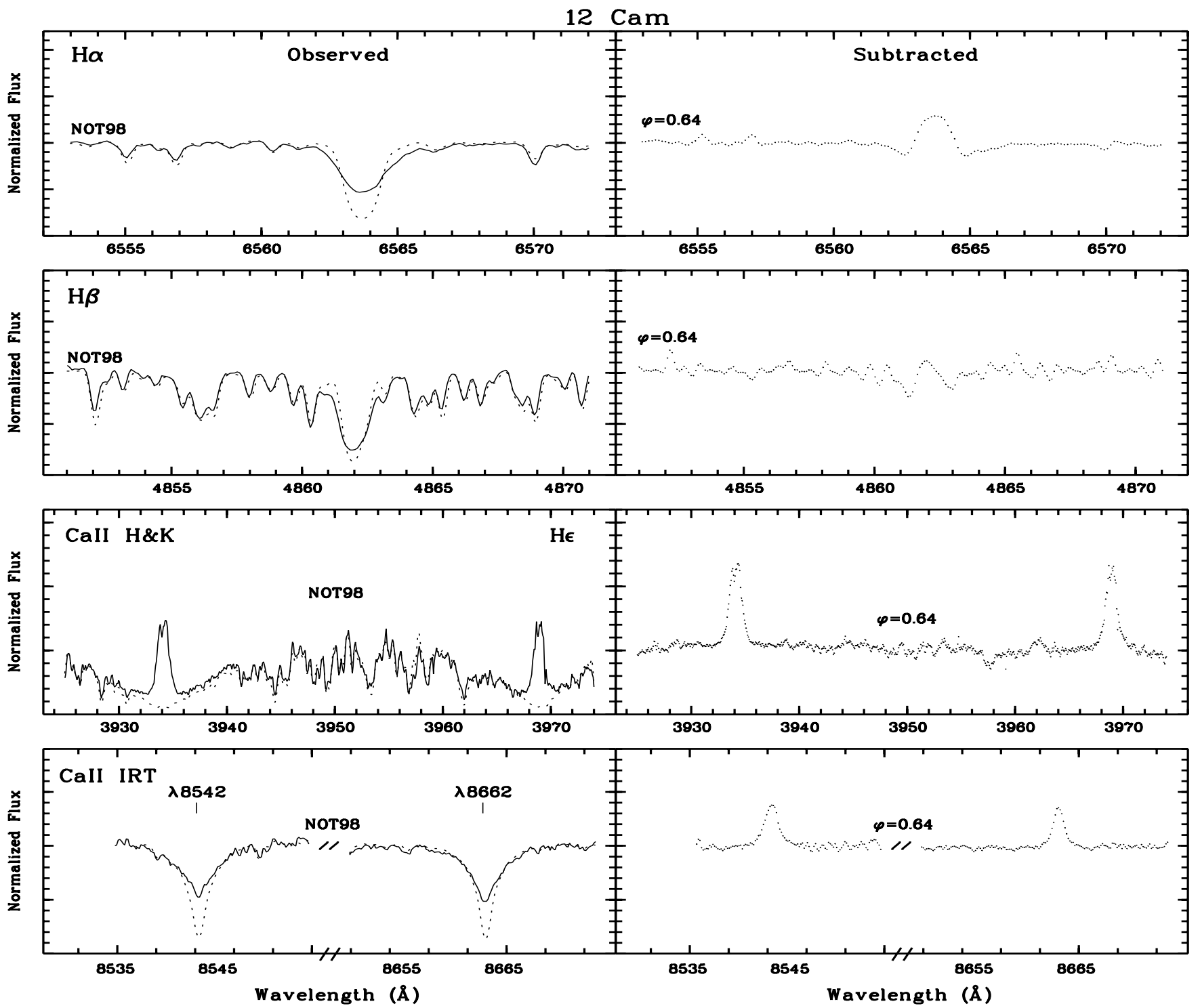

Fig. 2. $\mathrm{H} \alpha, \mathrm{H} \beta$, Ca II H \& K, and Ca II IRT spectra of 12 Cam. Observed and synthetic spectra in the left panel and subtracted spectra in the right panel

of the $\mathrm{H} \alpha$ line is in emission, confirming the high variability of the $\mathrm{H} \alpha$ line profile in this system. Strong emission is observed in the $\mathrm{Ca}$ II $\mathrm{H} \& \mathrm{~K}$ lines but the $\mathrm{H} \epsilon$ line is not detected. The Ca II IRT lines $(\lambda \lambda 8542,8662 \AA)$ show a strong filling-in. The $\mathrm{He} \mathrm{I}_{3}$ line appears in absorption (Fig. 24).

\subsection{OU Gem (HD 45088)}

OU Gem is a bright ( $V=6.79$, Strassmeier et al. 1990) and nearby $(d=14.7 \mathrm{pc}, \mathrm{ESA} 1997)$ BY Dra-type SB2 system $(\mathrm{K} 3 \mathrm{~V} / \mathrm{K} 5 \mathrm{~V})$ with an orbital period of 6.99 days and a noticeable eccentricity (Griffin \& Emerson 1975). Both components show Ca II H \& $\mathrm{K}$ emission, though the primary shows slightly stronger emission than the secondary. The $\mathrm{H} \alpha$ line is in absorption for the primary and filled-in for the secondary (Bopp 1980; Bopp et al. 1981a,b; Strassmeier et al. 1990; Montes et al. 1995a,b, 1996). Dempsey et al. (1993a) observed that the Ca II IRT lines were filled-in. This binary was detected by the WFC on board the ROSAT satellite during the all-sky survey (Pounds et al. 1993; Pye et al. 1995). OU Gem has $1.710^{29} \mathrm{erg} \mathrm{s}^{-1} \mathrm{X}$-ray luminosity, typical value of the BY Dra systems (Dempsey et al. 1993b, 1997). The photometric variability was discovered by Bopp et al. (1981a) and they also computed a 7.36-day photometric period. It is interesting that the orbital and rotational periods differ in $5 \%$ due to the appreciable orbital eccentricity $(e=0.15)$, according to Bopp (1980). Although BY Dra systems are main-sequence stars, their evolutionary stage is not clear. OU Gem has been listed by Soderblom et al. (1990) and Montes et al. (2000a, 2000c) as a possible member of the 


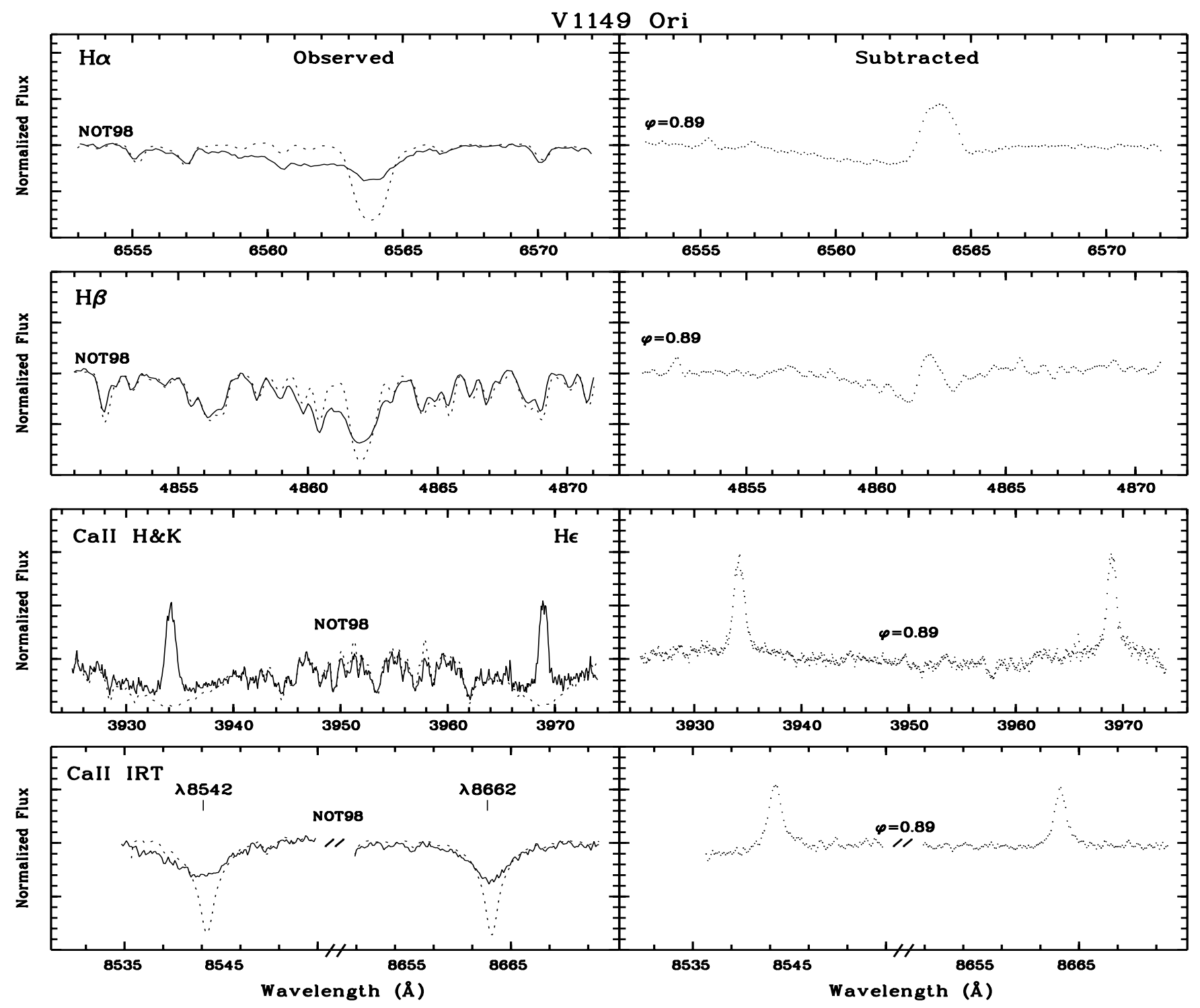

Fig. 3. $\mathrm{H} \alpha, \mathrm{H} \beta$, Ca II H \& K, and Ca II IRT spectra of V1149 Ori. Observed and synthetic spectra in the left panel and subtracted spectra in the right panel

UMa moving group (300 Myr), indicating that it may be a young star.

The Ho line: In the observed spectra, we see an absorption line for the primary star and a nearly complete filling-in for the secondary star. After applying the spectral subtraction technique, clear excess $\mathrm{H} \alpha$ emission is obtained for the two components, being stronger for the hot one (see Fig. 4 upper panel). The excess $\mathrm{H} \alpha$ emission $E W$ is measured in the subtracted spectrum and corrected for the contribution of the components to the total continuum. We took one spectrum in this region in Dec-92 (Montes et al. 1995b). At the orbital phase of this observation $(\varphi=0.48)$ we could not separate the emission from both components and we measured the total excess $\mathrm{H} \alpha$ emission $E W$ relative to the combined continuum. We obtained a similar value to Mar-96, Apr-98 and Jan-99 val- ues obtained adding up the excess emission $E W$ from the two components.

The $H \beta$ line: Looking at the observed spectra, we only see the $\mathrm{H} \beta$ line for the primary, in absorption. After applying the spectral subtraction technique small excess $\mathrm{H} \beta$ emission is obtained for the two components (see Fig. 4 lower panel). We have obtained, in general, $\frac{E_{\mathrm{H} \alpha}}{E_{\mathrm{H} \beta}}$ values larger than three for the two components, so the emission can come from prominences.

The $C a$ II $H$ \& $K$ and $H \epsilon$ lines: We observe that both components of this binary have the $\mathrm{Ca}$ II $\mathrm{H} \& \mathrm{~K}$ and $\mathrm{H} \epsilon$ lines in emission. We can also see that the excess Ca II $\mathrm{H}$ \& $\mathrm{K}$ emission of the hot star is larger than the one of the cool star (Fig. 5 upper panel). The measured excess Ca II $\mathrm{H} \& \mathrm{~K}$ emission of both components is larger in the two spectra of the NOT98 observing run than in the NOT96 

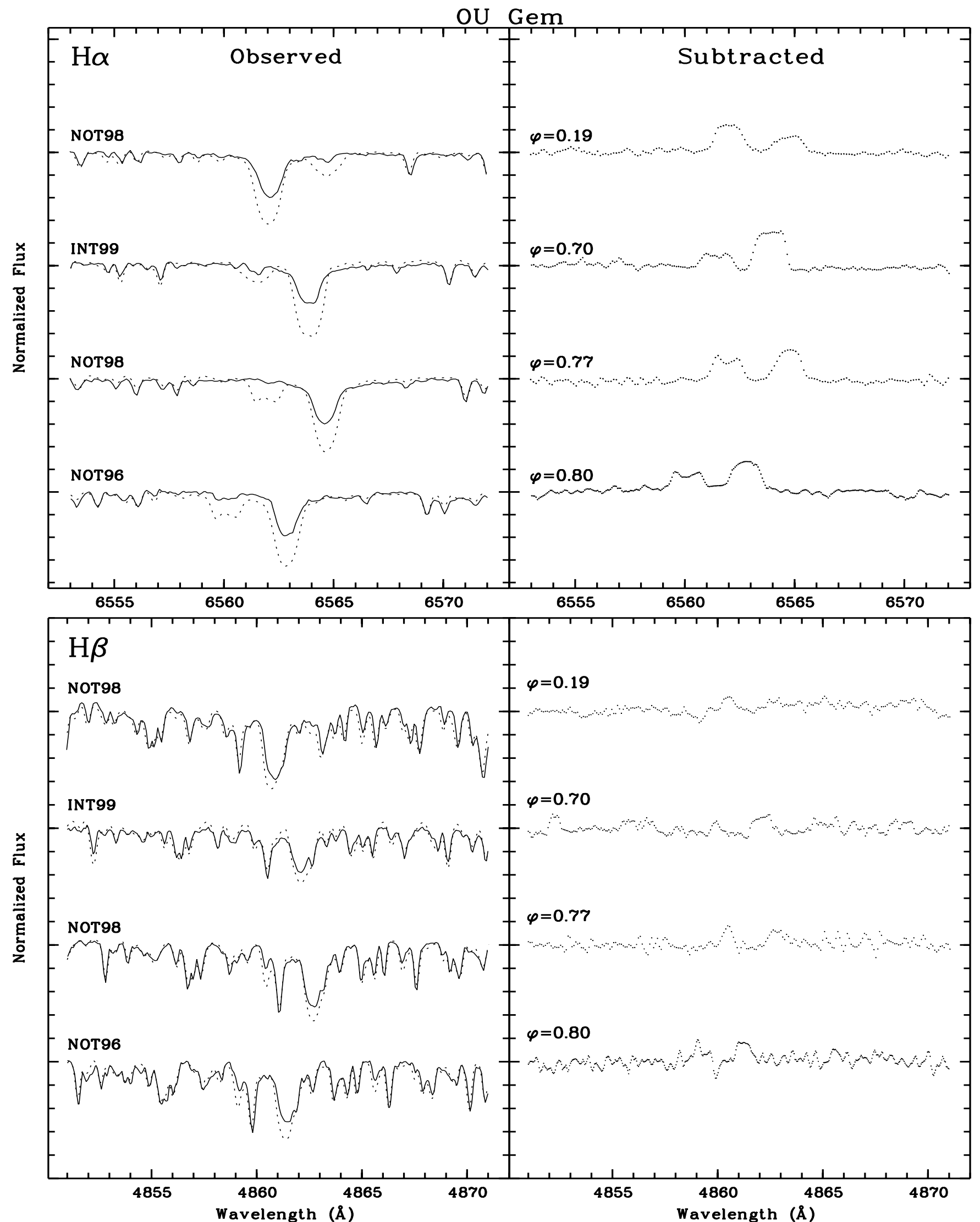

Fig. 4. $\mathrm{H} \alpha$ and $\mathrm{H} \beta$ spectra of $\mathrm{OU}$ Gem. Observed and synthetic spectra in the left panel and subtracted spectra in the right panel 
D. Montes et al.: Multiwavelength optical observations of chromospherically active binary systems. III.

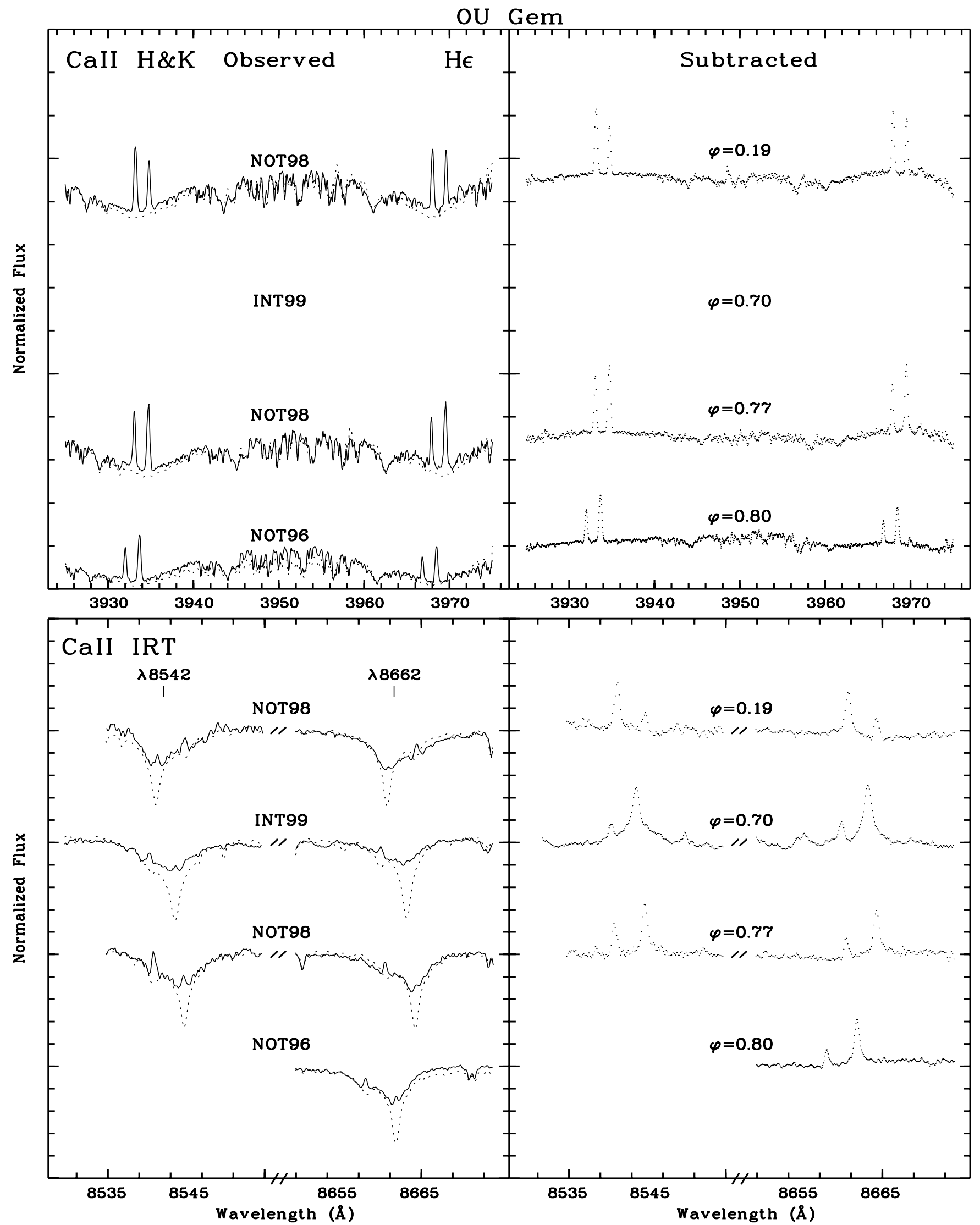

Fig. 5. Ca II H \& K and Ca II IRT spectra of OU Gem. Observed and synthetic spectra in the left panel and subtracted spectra in the right panel 

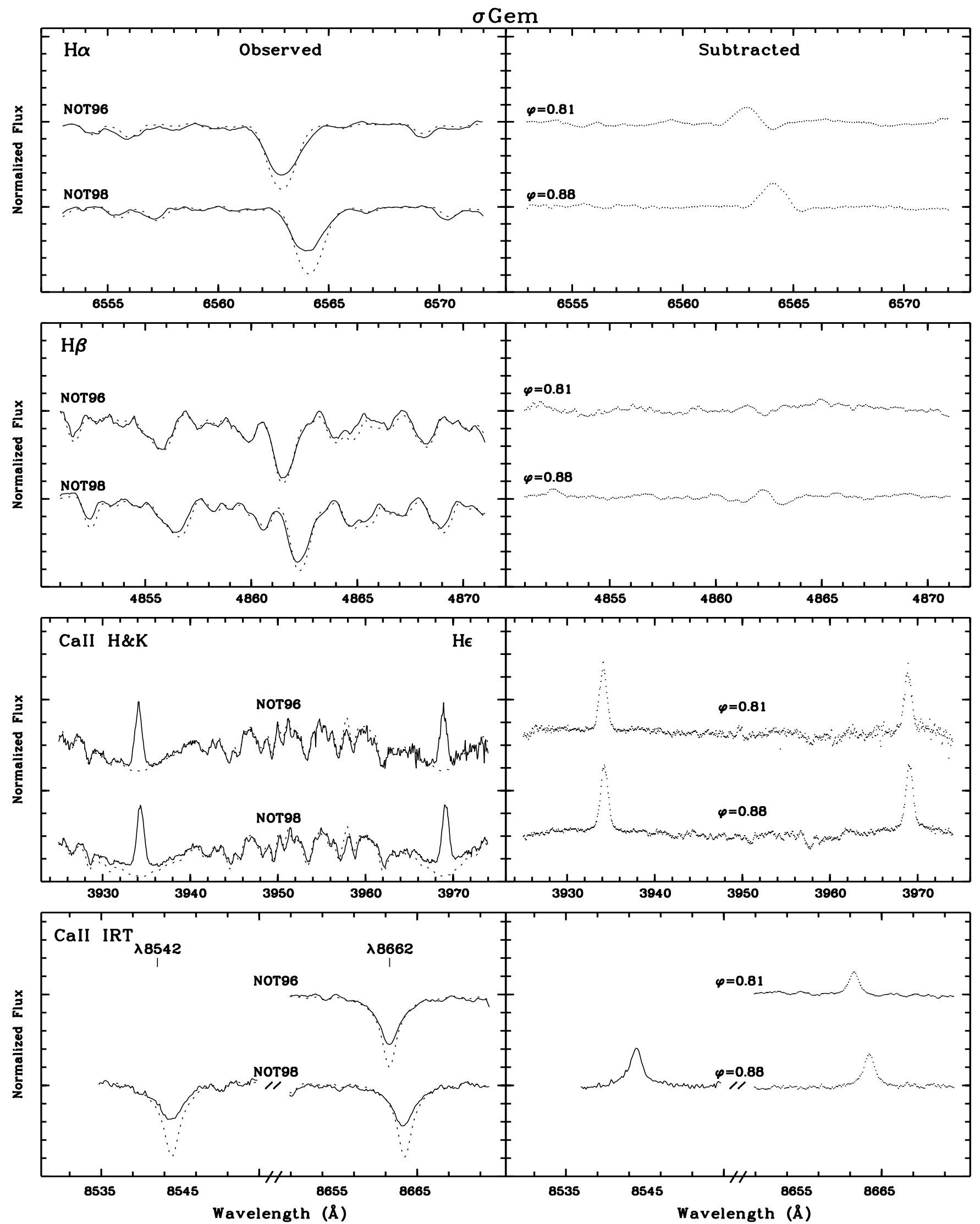

Fig. 6. $\mathrm{H} \alpha, \mathrm{H} \beta$, Ca II H \& $\mathrm{K}$, and Ca II IRT spectra of $\sigma$ Gem. Observed and synthetic spectra in the left panel and subtracted spectra in the right panel 


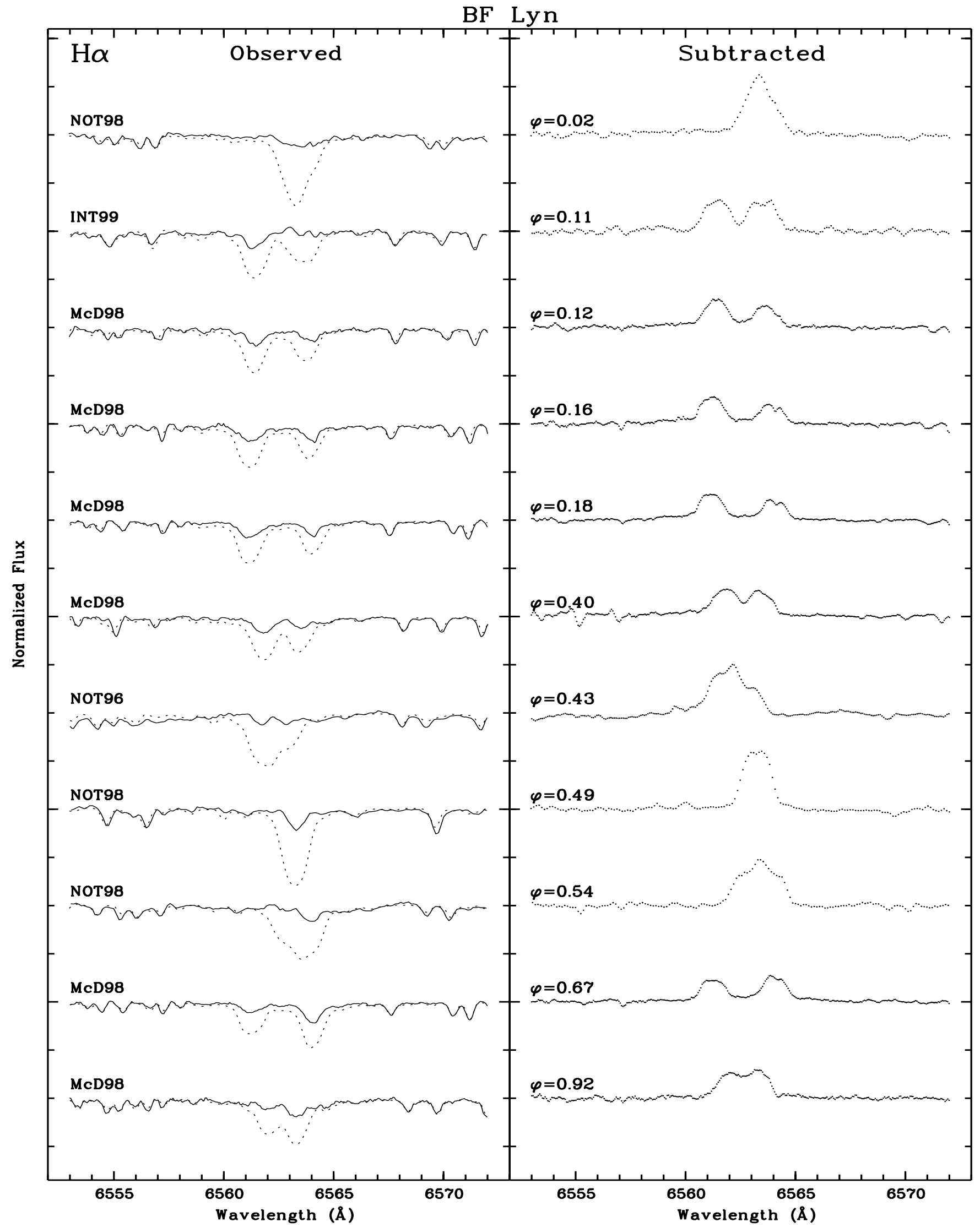

Fig. 7. H $\alpha$ observed (left panel) and subtracted (right panel) spectra of BF Lyn 

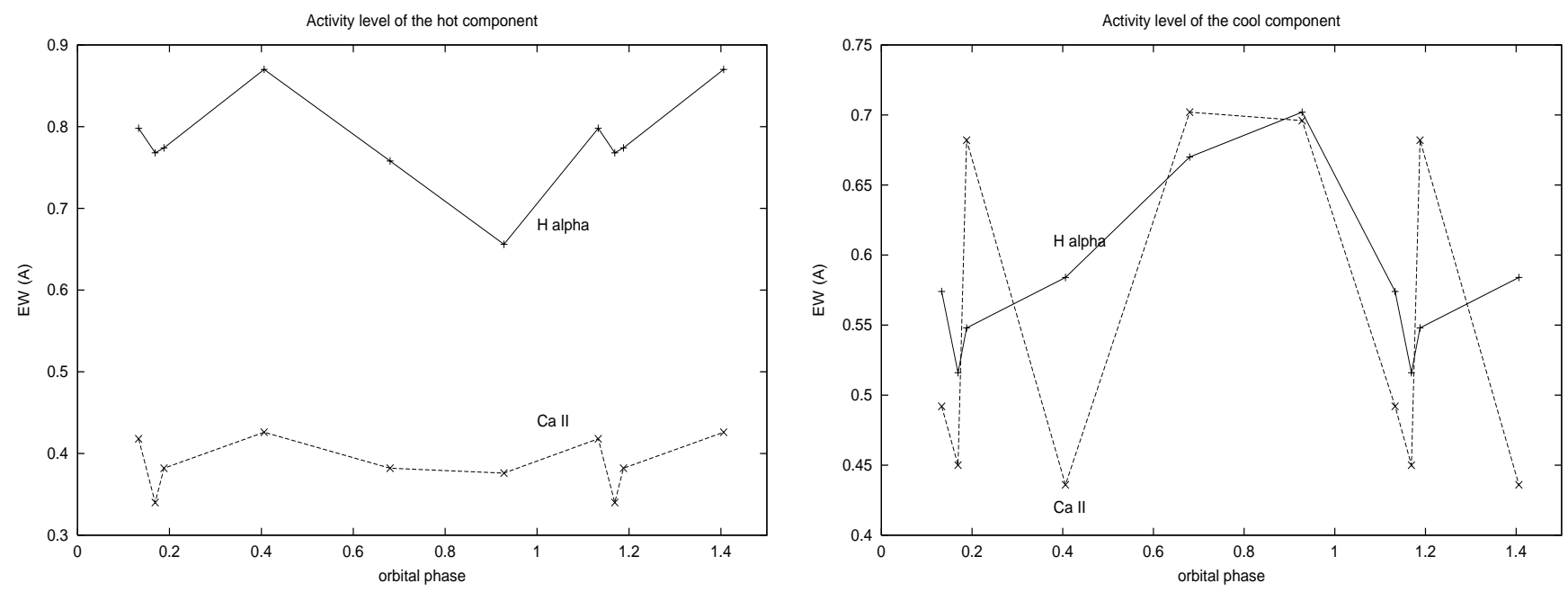

Fig. 8. $\mathrm{H} \alpha$ and Ca II IRT $\lambda 8542 \mathrm{EW}$ of the hot (left panel) and cool (right panel) components of BF Lyn for the McD98 run versus the orbital phase

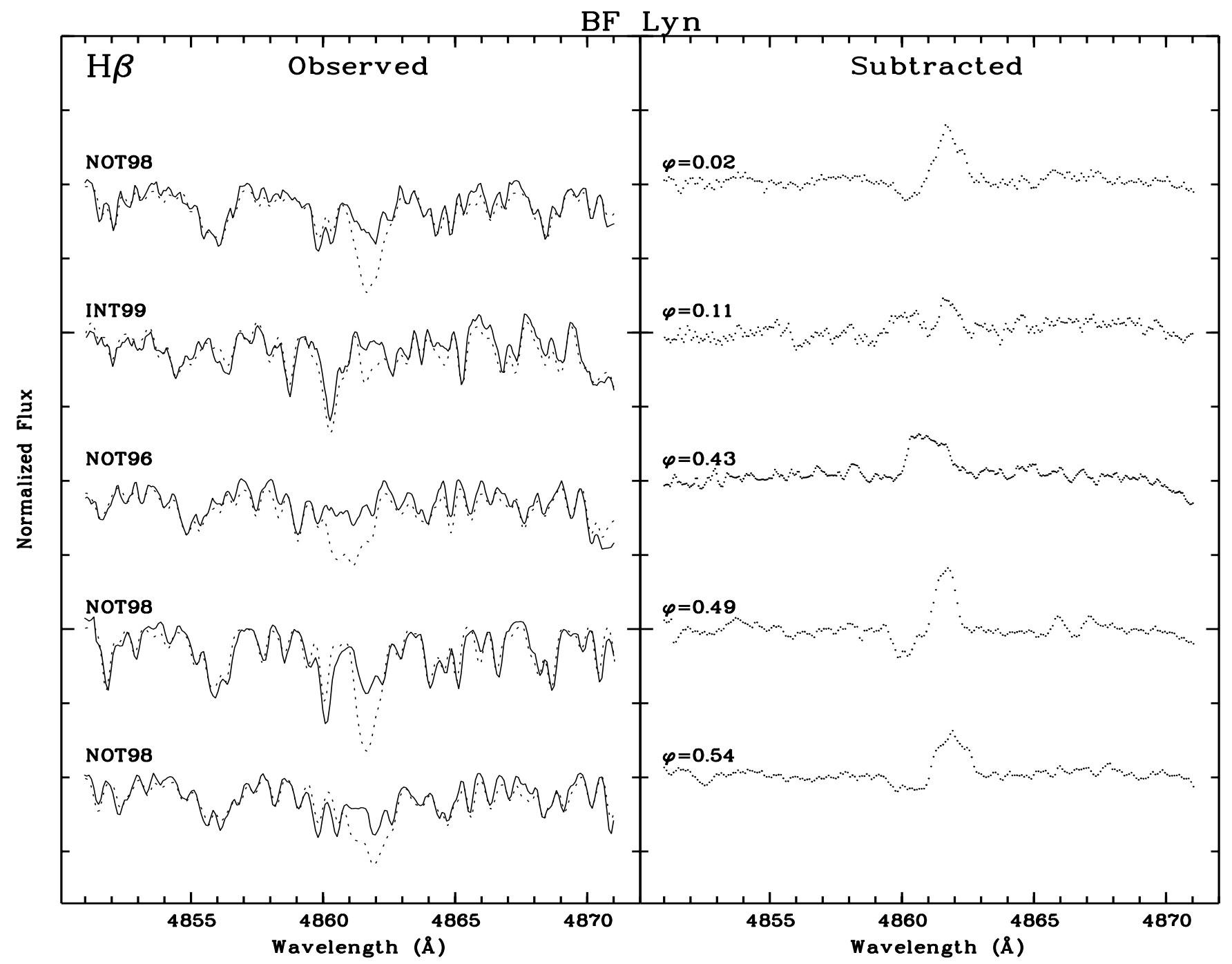

Fig. 9. $\mathrm{H} \beta$ observed (left panel) and subtracted (right panel) spectra of BF Lyn 


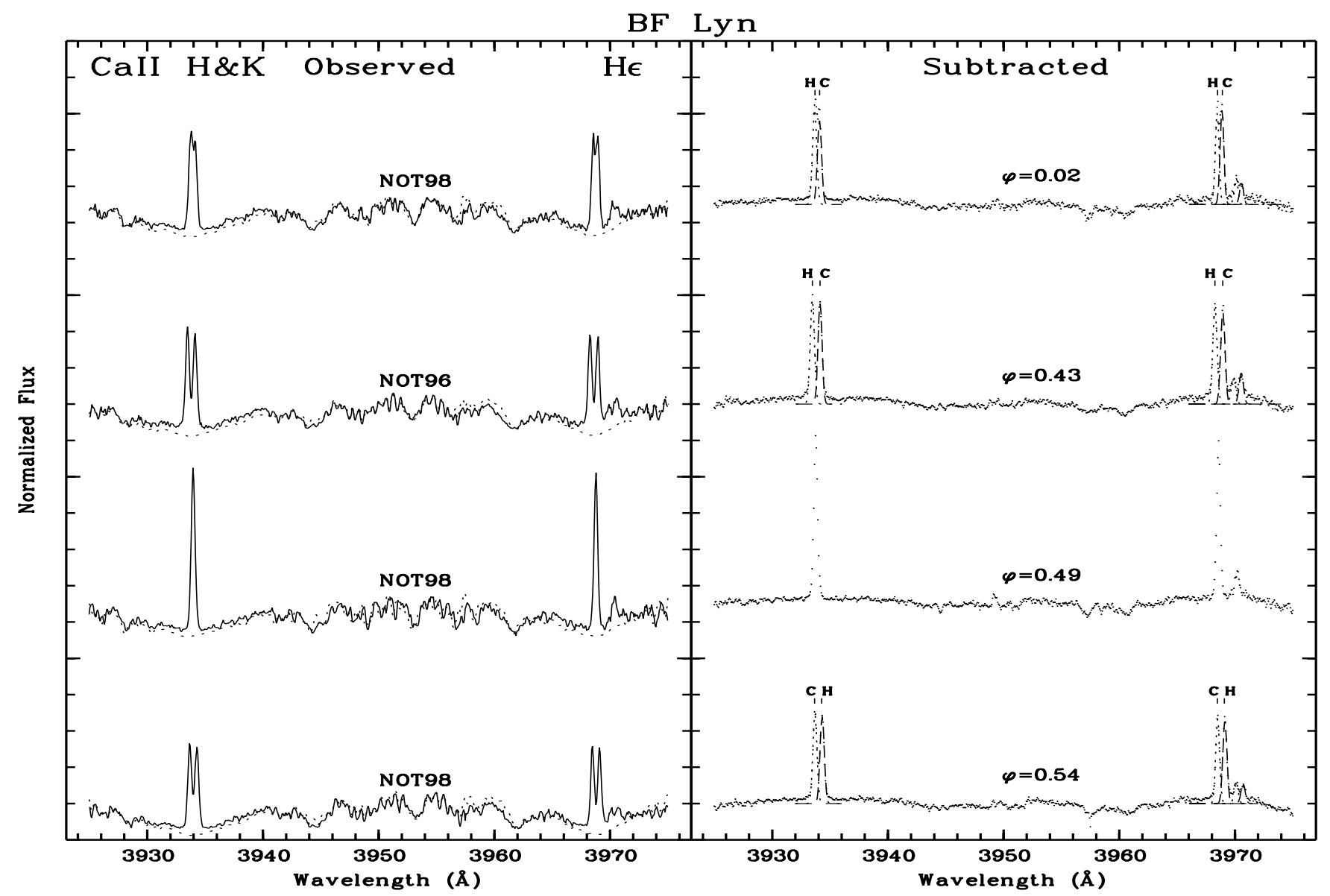

Fig. 10. Ca II H \& K observed (left panel) and subtracted (right panel) spectra of BF Lyn. We have also plotted the Gaussian fit to the subtracted spectrum used to deblend the contribution of the hot $(\mathrm{H})$ and cool $(\mathrm{C})$ components (short- and long-dashed lines)

spectrum. Overlapping between the $\mathrm{H} \epsilon$ line of one star and the $\mathrm{Ca}$ II $\mathrm{H}$ line of the other only allows to see the $\mathrm{H} \epsilon$ line of the cool star at orbital phase 0.19 , and $\mathrm{H} \epsilon$ of the hot star otherwise.

The Ca II IRT lines: In the observed spectra, we can see that both components of OU Gem show the Ca II IRT lines in emission superimposed to the corresponding absorption. After applying the spectral subtraction technique, clear excess emission appears for the two components, being clearly stronger for the hot one (see Fig. 5 lower panel).

\section{5. $\sigma$ Gem (HD 62044)}

This single-lined spectroscopic binary belongs to the longperiod group of RS CVn binary systems. It was classified as K1III, but the radius obtained by Duemmler et al. (1997) seems to be too small for a giant star. In Table 2 we have adopted the orbital and physical parameters updated by Duemmler et al. (1997). Strong and variable Ca II $\mathrm{H} \& \mathrm{~K}$ emission always centered at the absorption line has been reported by Bopp (1983), Strassmeier et al. (1990),
FFMCC, Montes 1995, Montes et al. 1996b. Our previous observation in the $\mathrm{H} \alpha$ line region (Montes et al. 1995a,b) revealed small excess emission, similar to that reported by Strassmeier et al. (1990) and Frasca \& Catalano (1994). Variable excess $\mathrm{H} \alpha$ emission anti-correlated with spot regions has been found by Zhang \& Zhang (1999).

The new observations (NOT96, NOT98) show strong $\mathrm{Ca}$ II $\mathrm{H} \& \mathrm{~K}$ emission lines and small emission in the $\mathrm{H} \epsilon$ line. After applying the spectral subtraction, a small filling-in is observed in the $\mathrm{H} \alpha, \mathrm{H} \beta$ and $\mathrm{Ca}$ II IRT lines (Fig. 6). We observe in this system notable He I $\mathrm{D}_{3}$ absorption (Fig. 24). All the activity indicators show an increase in the 1998 observation $(\varphi=0.88)$ with respect to 1996 one $(\varphi=0.81)$. The emission in the Ca II H \& K lines in these two observations is noticeably larger than in our previous observations at different epochs and orbital phases.

\subsection{BF Lyn (HD 80715)}

This double-lined spectroscopic binary with spectral types $\mathrm{K} 2 \mathrm{~V} /[\mathrm{dK}]$ shows variable $\mathrm{H} \alpha$ emission and strong 


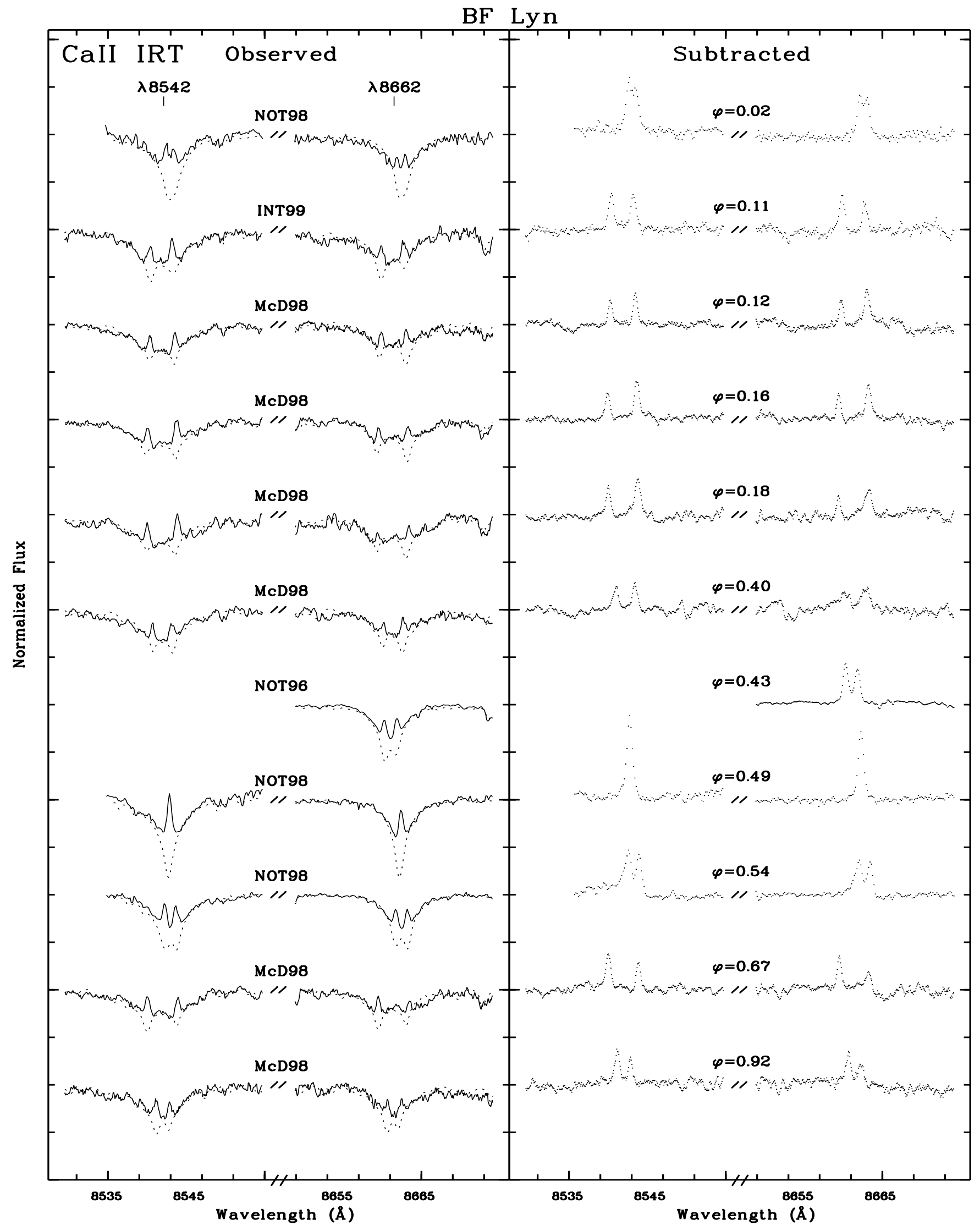

Fig. 11. Ca II IRT $\lambda 8542$ and $\lambda 8662$ observed (left panel) and subtracted (right panel) spectra of BF Lyn 

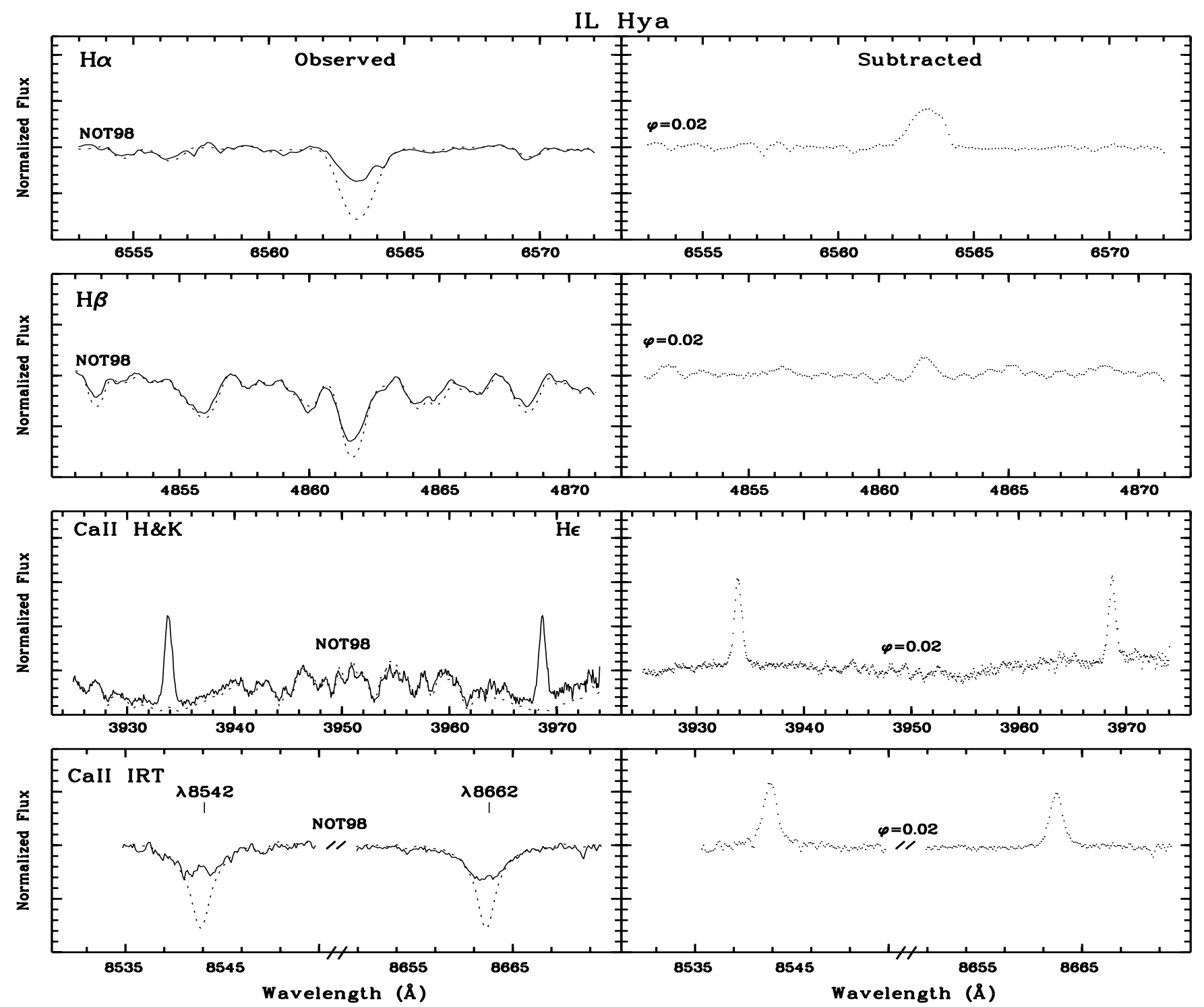

Fig. 12. $\mathrm{H} \alpha, \mathrm{H} \beta$, Ca II H \& K, and Ca II IRT spectra of IL Hya. Observed and synthetic spectra in the left panel and subtracted spectra in the right panel

$\mathrm{Ca}$ II $\mathrm{H} \& \mathrm{~K}, \mathrm{H} \epsilon$ and $\mathrm{Ca}$ II IRT emission from both components (Barden \& Nations 1985; Strassmeier et al. 1989b). In our previous observation (Montes et al. 1995c) we found strong emission in the $\mathrm{Ca}$ II $\mathrm{H} \& \mathrm{~K}$ lines from both components with very similar intensity and the $\mathrm{H} \epsilon$ line in emission. The orbital period is 3.80406 days (Barden \& Nations 1985), and Strassmeier et al. (1989b), from photometric observations, found that BF Lyn is a synchronized binary with a circular orbit.

In the four runs analysed in this paper we have obtained 11 spectra of BF Lyn at different orbital phases. We have used the original heliocentric Julian date on conjunction $\left(T_{\text {conj }}\right)$ given by Barden \& Nations (1985) to calculate the orbital phases since we discovered a mistake in the Strassmeier et al. (1993) catalog where the orbital data from Barden \& Nations (1985) are compiled. In the original paper the epoch was determined using Modified Julian Date (MJD), that is why 0.5 days must be added to the Strassmeier et al. (1993) date, who used the 2440000.0 Julian day as a reference date.

The Ho line: We took several spectra of BF Lyn in the $\mathrm{H} \alpha$ line region in four different epochs and at different orbital phases. In all the spectra (Fig. 7) we can see the $\mathrm{H} \alpha$ line in absorption from both components. The spectral subtraction reveals that both stars have excess $\mathrm{H} \alpha$ emission. At some orbital phases, near to the conjunction, it is impossible to separate the contribution of both components. The excess $\mathrm{H} \alpha$ emission of BF Lyn shows variations with the orbital phase for both components, but the hot star is the most active in $\mathrm{H} \alpha$. In Fig. 8 we have plotted for the McD 98 observing run the excess $\mathrm{H} \alpha$ emission $E W$ versus the orbital phase for the hot and cool components. 

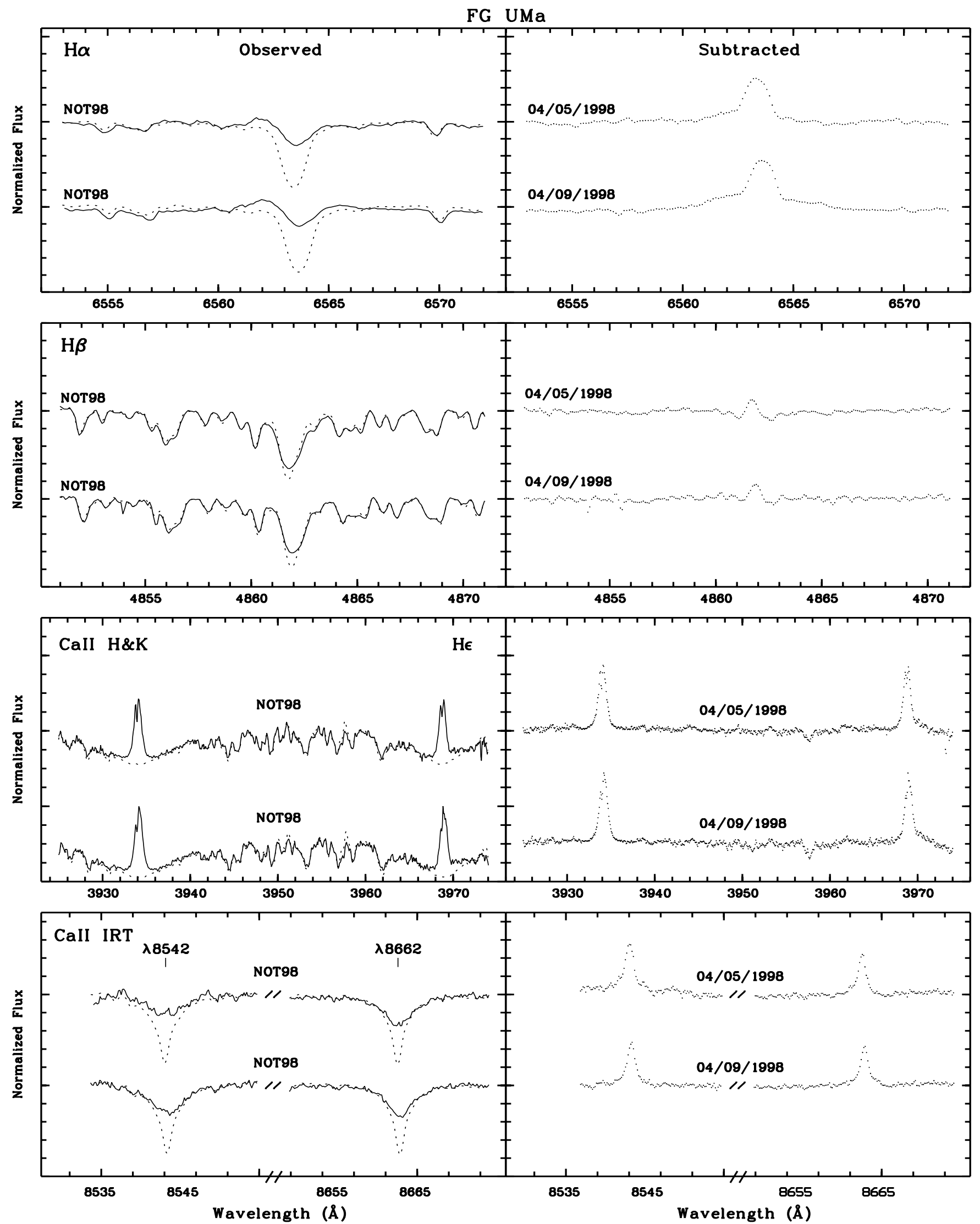

Fig. 13. $\mathrm{H} \alpha, \mathrm{H} \beta$, Ca II H \& K, and Ca II IRT spectra of FG UMa. Observed and synthetic spectra in the left panel and subtracted spectra in the right panel 


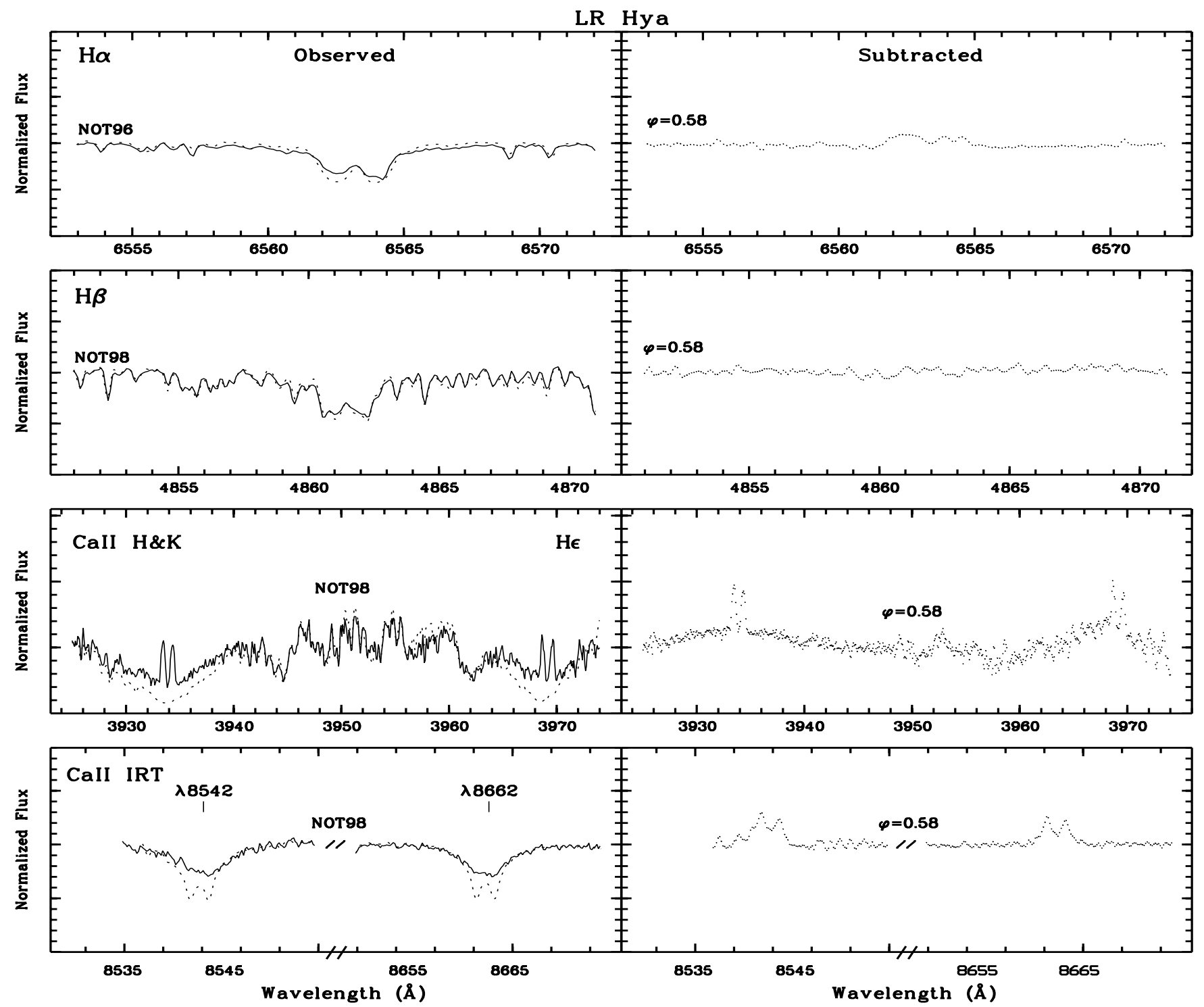

Fig. 14. $\mathrm{H} \alpha, \mathrm{H} \beta$, Ca II H \& K, and Ca II IRT spectra of LR Hya. Observed and synthetic spectra in the left panel and subtracted spectra in the right panel

The highest excess $\mathrm{H} \alpha$ emission $E W$ for the hot component has been reached at about 0.4 orbital phase and the lowest value is placed at about 0.9 orbital phase, whereas the cool component shows the highest excess $\mathrm{H} \alpha$ emission $E W$ at near 0.9 orbital phase and the lowest value between 0.2 and 0.4 orbital phases. The variations of the excess $\mathrm{H} \alpha$ emission $E W$ for both components are anti-correlated, which indicates that the chromospheric active regions are concentrated on faced hemispheres of both components, but at about 0.4 and 0.9 orbital phases for the hot and cool component, respectively. The same behaviour is also found in Ca II IRT. The excess $\mathrm{H} \alpha$ emission $E W$ also shows seasonal variations, for instance, the values of the INT99 observing run are very different, specially for the cool component, from McD 98 values at very similar orbital phase.
The $H \beta$ line: Five spectra in the $\mathrm{H} \beta$ line region are available. In all of them the $\mathrm{H} \beta$ line appears in absorption from both components. The application of the spectral subtraction technique reveals clear excess $\mathrm{H} \beta$ emission from both stars (see Fig. 9). The $\left(\frac{E_{\mathrm{H} \alpha}}{E_{\mathrm{H} \beta}}\right)$ values that we have found for this star allow us to say that the emission comes from extended regions viewed off the limb.

The $C a$ II $H \& K$ and $H \epsilon$ lines: We took four spectra in the Ca II H \& $\mathrm{K}$ region during the NOT (96 \& 98) observing runs (Fig. 10). Another spectrum was taken in 1993 with the $2.2 \mathrm{~m}$ telescope at the German Spanish Astronomical Observatory (CAHA) (Montes et al. 1995c). These spectra exhibit clear and strong Ca II $\mathrm{H} \& \mathrm{~K}$ and $\mathrm{H} \epsilon$ emission lines. At $0.02,0.43$, and 0.54 orbital phases the emission from both components can be deblended using a two-Gaussian fit (see Fig. 10). In the case of CAHA 93 
run, the $\mathrm{H} \epsilon$ emission line from the hot component is overlapped with the $\mathrm{Ca}$ II $\mathrm{H}$ emission of the cool component. The excess $\mathrm{Ca}$ II $\mathrm{H} \& \mathrm{~K}$ and $\mathrm{H} \epsilon$ emission changes with the orbital phase during the NOT 98 run in the same way as the corresponding excess Ca II $\lambda 8542$ and $\mathrm{H} \alpha$ emission. The excess Ca II H \& K emission $E W$ also shows seasonal variations, for instance, the values of CAHA 93 observing run are lower than NOT $96 \& 98$ values.

The $C a$ II IRT lines: In all the spectra we can see the Ca II IRT lines in emission from both components (Fig. 11). As in the case of $\mathrm{H} \alpha$, the Ca II IRT emission shows variations with the orbital phase for both components. In Fig. 8 we have plotted, for the McD 98 observing run, the excess Ca II $\lambda 8542$ emission $E W$ versus the orbital phase for the hot and cool components. The variations of the excess Ca II emission $E W$ for both components are anti-correlated and they show the same behaviour as the excess $\mathrm{H} \alpha$ emission $E W$.

\subsection{IL Hya (HD 81410)}

IL Hydrae is a typical RS CVn star with very strong Ca II $\mathrm{H} \& \mathrm{~K}$ emission (Bidelman \& MacConnell 1973). The 12.86833-day photometric period derived by Raveendran et al. (1982) is very close to the orbital period. It was found to be an X-ray source and a microwave emitter (Mitrou et al. 1996). From a photometric analysis, Cutispoto (1995) estimated the secondary to be a G8V star. Later, Donati et al. (1997) detected the secondary component in the optical range and they calculated a $1.0 R_{\odot}$ radius for it. Weber \& Strassmeier (1998) gave a K0III-IV type for the primary and computed the first double-lined orbit of IL Hya. Later, Raveendran \& Mekkaden (1998) gave a new orbital solution and just recently, Fekel et al. (1999) have presented updated SB2 orbital elements which we have adopted and they are given in Table 2 (we have corrected the heliocentric Julian date on conjunction $\left(T_{\text {conj }}\right)$ so that the primary is in front). The multiwavelength Doppler images presented by Weber \& Strassmeier (1998) revealed a cool polar spot and several features at low latitudes. These authors also found that the $\mathrm{H} \alpha E W$ showed sinusoidal variation which was in phase with the photospheric light curve.

We have taken one spectrum of IL Hya (NOT98) with 0.02 orbital phase which is very close to the conjunction, so the very weak lines of the secondary can not be detected in any wavelength. In the observed spectrum (Fig. 12), the $\mathrm{H} \alpha$ line can be seen as a filled-in absorption line with a superimposed $1.04 \AA\left(48 \mathrm{~km} \mathrm{~s}^{-1}\right)$ red-shifted absorption feature, as obtained from a two-Gaussian fit. After applying the spectral subtraction, clear excess emission is observed. The excess emission shows an asymmetric profile due to the red-shifted absorption feature. Similar $\mathrm{H} \alpha$ profiles were observed by Weber \& Strassmeier (1998) in this system, but the red-shifts measured in their spectra were larger $(1.24 \AA)$ and remained constant during a rotational cycle. This behaviour could be due to mass motions that change from one epoch to another, but a combination of several dynamical processes may be involved. A filling-in is also found in the $\mathrm{H} \beta$ line. According to the value obtained for the corrected ratio of the excess emission $E W$ of both lines, the emission may be ascribed to an extended region viewed off the limb. The $\mathrm{He} \mathrm{I}_{3}$ line appears in absorption (Fig. 24), but no filling-in is detected in the $\mathrm{Na}$ I $\mathrm{D}_{1}, \mathrm{D}_{2}$ lines. The $\mathrm{Ca}$ II $\mathrm{H} \& \mathrm{~K}$ lines are observed in emission. Furthermore, the Ca II IRT lines show clear central emission reversal.

\subsection{FG UMa (HD 89546)}

FG UMa is the least studied star of our sample. Bidelman (1981) included it in his Catalogue of stars with Ca II H \& $\mathrm{K}$ emission. This star is identified as a single-lined binary by CABS. A 21.50-day photometric period has been obtained from the automated monitoring that Henry et al. (1995a) carried out. From spectroscopic measurements, they confirmed $V \sin i=(15 \pm 2) \mathrm{km} \mathrm{s}^{-1}$ and a G8IV spectral type. These authors also mentioned that, according to an unpublished orbital analysis, the system is synchronized and circularized. We have also taken from them the orbital period and the radius. Some indication of possible eclipses is noted in the Hipparcos Catalogue (ESA 1997). Fluxes of the Ca II H \& K emission lines have been calculated by Strassmeier (1994b) and a filled-in and variable $\mathrm{H} \alpha$ line has been reported by Henry et al. (1995a).

We have not got enough data to be able to compute the orbital phases corresponding to the two spectra (NOT98) that we present here. However, there is a change of 0.2 in the photometric phase between both observations. We have not found any evidence of the secondary star through the whole spectral range. Moreover, according to the appearance of some Ti I and Fe I lines (Paper II) we suggest that the observed spectra correspond to a luminosity class more evolved than subgiant (in agreement with the radius calculated by Henry et al. 1995a, who suggested a luminosity class III-IV). The presence of a notable He I $\mathrm{D}_{3}$ absorption line (Fig. 24) encourages this conclusion. In the observed spectra of the $\mathrm{H} \alpha$ and $\mathrm{H} \beta$ lines (Fig. 13), both absorption lines show a clear filling-in. The spectral subtraction allows us to compare the two observations. As it can be read in Table 5 the ratio of the excess emission $E W$ is typical of extended regions viewed off limb, and a significant variation, mainly due to $\mathrm{H} \beta$, is obtained for the two different nights. Furthermore, excess emission is detected in the blue wing of the $\mathrm{H} \alpha$ line. Similar behaviour was mentioned by Henry et al. (1995a). Strong filling-in is observed in the Ca II IRT lines. The Ca II H \& $\mathrm{K}$ lines present strong emission with clear self-absorption with blue-shifted asymmetry in both observations. 


\subsection{LR Hya (HD 91816)}

It is a double-lined spectroscopic binary, classified as a BY Dra-type system, that contains two almost identical K-type dwarf components (Bopp et al. 1984). The orbital parameters were determined by Fekel et al. (1988) who suggested a K0V spectral type for both components. The photometric period of 3.1448 days, given in CABS, was reported by Bopp et al. (1984), but following observation campaigns could not confirm that value. In fact, the results obtained are not consistent (Strassmeier 1989; Cutispoto 1991, 1993) and point out low-amplitude rotational modulation due to the development and decline of small active regions at different longitudes of both components.

We only have got one spectrum (NOT98) of this system at 0.58 orbital phase, so that the chromospheric activity indicators from both components can be easily analyzed. The $\mathrm{H} \alpha$ absorption line (Fig. 14) shows a weak filling-in for both components, as it has been previously mentioned by Strassmeier et al. (1990). No filling-in is detected in the $\mathrm{H} \beta$ line. Although the $S / N$ ratio in the $\mathrm{Ca}$ II $\mathrm{H} \& \mathrm{~K}$ region is very low in this observation and the synthetic and observed spectra are not well matched, we can clearly see moderate emission in the $\mathrm{Ca}$ II $\mathrm{H} \&$ $\mathrm{K}$ lines from both components. The Ca II IRT lines of the two stars exhibit a clear filling-in. The measured excess emission in the different lines are very similar in both components, although a bit larger in the red-shifted one.

\subsection{0. $H U$ Vir (HD 106225)}

HU Vir is a late-type star (KOIII-IV) with strong Ca II H \& K emission noted by Bidelman (1981) for the first time. Recently, Fekel et al. (1999) have discovered that HU Vir is a triple system with a long period of about 6.3 years and we have taken from them the spectral type, $T_{\text {conj }}$ and the rotational period. The $B-V$ colour index has been taken from Hipparcos Catalogue (ESA 1997) and $V \sin i$ from Fekel (1997). Fekel et al. (1986) observed the $\mathrm{H} \alpha$ line in emission and Strassmeier \& Fekel (1990) found the H $\epsilon$ line in emission. Such emission lines are seen in the most active RS CVn type systems. Strassmeier (1994a) found a big, cool polar spot from Doppler imaging and two hot plages $180^{\circ}$ apart from the $\mathrm{H} \alpha$ and $\mathrm{Ca}$ II line-profile analysis. The chromospheric plages seemed to be spatially related to two large appendages of the polar spot. Broadening in the $\mathrm{H} \alpha$ profile suggested mass flow in a coronal loop connecting the two plage regions. Hatzes (1998) used the Doppler imaging technique to derive the cool spot distribution. He found a large spot at latitude $45^{\circ}$ and a weak polar spot with an appendage. The polar spot was considerably smaller than similar features found on other RS $\mathrm{CVn}$ stars. From an analysis of the $\mathrm{H} \alpha$ variations he also found evidence for a plage located at high $\left(\approx 70^{\circ}\right)$ latitude, near the polar extension.
The Ho line: Strassmeier (1994a) identified three distinctive features in the $\mathrm{H} \alpha$ line: blue-shifted emission, central sharp absorption and red-shifted broad absorption. Hatzes (1998) found similar behaviour in this line. In our spectra (Fig. 15), the $\mathrm{H} \alpha$ line always appears in emission, although its intensity is variable. Moreover, the emission is blue-shifted at some orbital phases. Hall \& Ramsey (1992, 1994) explained the blue-shifted emission as prominencelike structures. We can see red-shifted broad absorption at the 0.54 orbital phase but we never observe central sharp absorption. Walter \& Byrne (1998) said that there was growing evidence for continuous low-level mass in-fall, seen as red-shifted absorption in $\mathrm{H} \alpha$ line profiles. We can also notice that the subtracted $\mathrm{H} \alpha$ profiles have broad and variable extended wings which are not well matched using a single-Gaussian fit. These profiles have been fitted using two Gaussian components. The parameters of the broad and narrow components used in the two-Gaussian fit are given in Table 4 and the corresponding profiles are plotted in the right panel of Fig. 15. These broad wings are observed at different orbital phases and in different epochs. In some cases the blue wing is noticeable stronger than the red wing and the fit is better matched when the broad component is blue-shifted with respect to the narrow component. We have interpreted these broad components as microflaring activity that occurs in the chromosphere of this very active star (Papers I, II; Montes et al. 1998b). The contribution of the broad component to the total $E W$ of the line ranges from $32 \%$ to $66 \%$ which is in the range observed in the stars analysed in Papers I and II. Strassmeier (1994a), in 1991, and Hatzes (1998), in 1995, observed strong emission between the 0.27 and 0.51 orbital phases. We have obtained the strongest emission at the 0.44 orbital phase, in January 1998, and at 0.29 , in April 1998 (see Table 3). Thus, our $0.29-0.44$ orbital phase interval is similar to Strassmeier and Hatzes's orbital phase interval, so we can conclude that HU Vir has an active longitude area (which corresponds to that orbital phase interval) since 1991. Similar active longitudes have been found by other authors in several chromospherically active binaries (Oláh et al. 1991; Henry et al. 1995b; Jetsu 1996; Berdyugina \& Tuominen 1998).

The $H \beta$ line: A nearly complete filling-in is observed (Fig. 16 upper panel). After applying the spectral subtraction technique, clear excess $\mathrm{H} \beta$ emission is obtained. We have obtained $\frac{E_{\mathrm{H} \alpha}}{E_{\mathrm{H} \beta}}$ values larger than three (see Table 5), so the emission can come from prominences.

The $\mathrm{Ca}$ II $\mathrm{H} \& \mathrm{~K}$ and $\mathrm{H \epsilon}$ lines: We can observe very strong $\mathrm{Ca}$ II $\mathrm{H} \& \mathrm{~K}$ emission and an important $\mathrm{H} \epsilon$ emission line superimposed to the wide Ca II H line (Fig. 16 lower panel). The $\mathrm{H} \epsilon$ line in emission indicates that $\mathrm{HU}$ Vir is a very active system. Similar strong emission was found in our previous observation obtained in March 1993 at 0.71 orbital phase (Montes et al. 1995c). We can also notice that the largest values of the excess Ca II H \& K emission $E W$ appear at the 0.29 and 0.38 orbital phases 


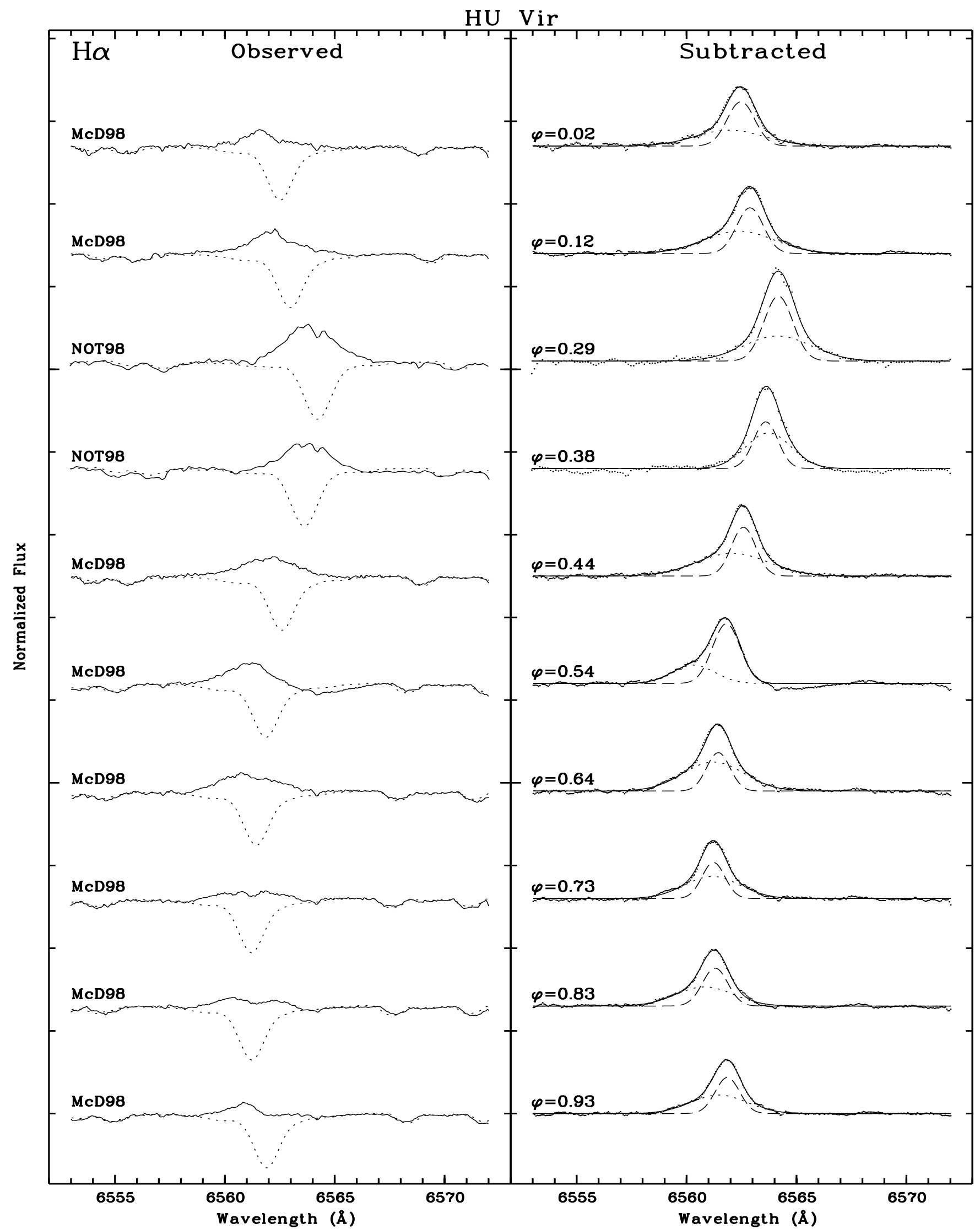

Fig. 15. H $\alpha$ observed (left panel) and subtracted (right panel) spectra of HU Vir. We have superposed the two Gaussian components fit (solid-line). The sort-dashed-line represents the broad component and the large-dashed-line the narrow one 


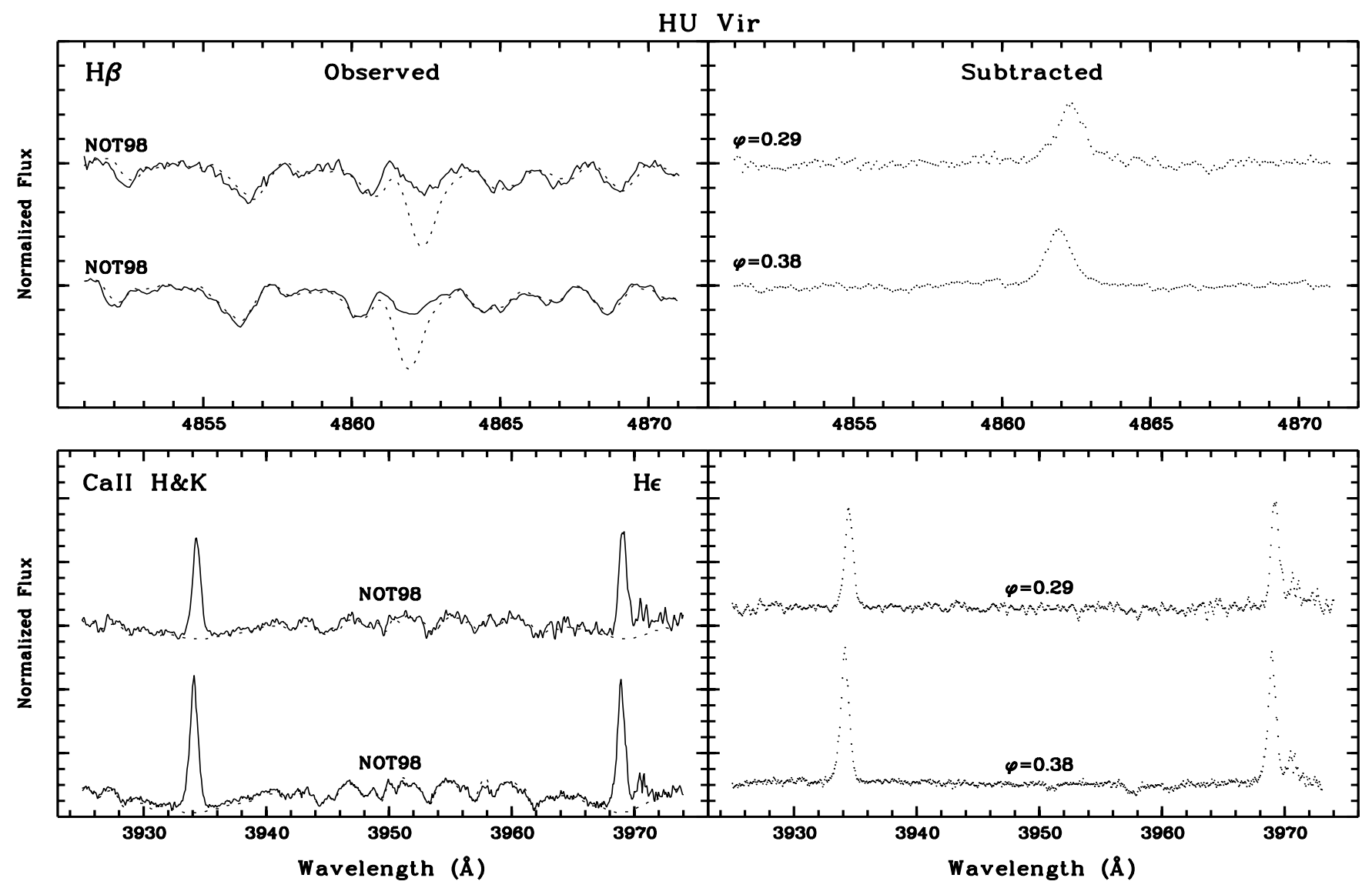

Fig. 16. $\mathrm{H} \beta$, and Ca II H \& K observed (left panel) and subtracted (right panel) spectra of HU Vir

(see Table 6). It is in agreement with the $\mathrm{H} \alpha$ line behaviour.

The Ca II IRT lines: HU Vir shows the Ca II IRT lines in emission above the continuum (Fig. 17). We observe that the emission is centered at its corresponding absorption. We also notice that the subtracted profiles have broad wings due to microflares according to Montes et al. (1997). The excess Ca II $\lambda 8542$ emission $E W$ (see Table 7) behaves like the excess $\mathrm{H} \alpha$ emission $E W$.

The He I $D_{3}$ line: We have not detected any significant absorption or emission for $\mathrm{He}_{\mathrm{I}} \mathrm{D}_{3}$ (Fig. 24), contrary to the absorption observed in other giants. This total fillingin of the He $\mathrm{I}_{3}$ line could be explained (Saar et al. 1997; Paper II) as a filling-in due to the low-level flaring (microflares) that takes place in this very active star according to the $\mathrm{H} \alpha$ broad component that we have found.

The $L i$ I $\lambda 6707.8$ line: The Li I absorption line is clearly observed in the eight spectra of the McD98 observing run (see Fig. 25). The mean $E W$ obtained is $56 \pm 11$ (mA). At this spectral resolution the $\mathrm{Li}$ I line is blended with the nearby Fe I $\lambda 6707.41 \AA$ line. We have corrected the total $E W$ measured, $E W(\mathrm{Li}$ I + Fe I $)$, by subtracting the $E W$ of Fe I calculated from the empirical relationship with $(B-V)$ given by Soderblom et al. (1990) $(E W(\mathrm{Fe} \mathrm{I})=$ $24(\mathrm{~m} \AA))$. Finally, the corrected $E W(\mathrm{Li} \mathrm{I})_{\mathrm{corr}}=32(\mathrm{~m} \AA)$ was converted into abundances by means of the curves of growth computed by Pallavicini et al. (1987), obtaining $\log N(\mathrm{Li}$ I $)=1.2($ on a scale where $\log N(\mathrm{H})=12.0)$ with an accuracy of the $\approx 0.30$ dex. This value is larger than the lower limit reported by Barrado et al. (1998) for this star.

\subsection{DK Dra (HD 1066r7)}

This is a double-lined spectroscopic binary with almost identical components of spectral types K1III and Ca II H \& K emission from both components (Bopp et al. 1979; Fekel et al. 1986; Strassmeier 1994b). Eker et al. (1995) reported variable nature of $\mathrm{H} \alpha$ and, using a subtracted spectrum, found emission of similar intensity from both components. In our previous observations (Montes et al. 1995a,b; FFMCC) we found a broad excess $\mathrm{H} \alpha$ emission line and the Ca II H \& $\mathrm{K}$ lines in emission, but all of them were taken at orbital phases near to the conjunction, so it was impossible to distinguish the contribution of each component.

The spectra analysed in this paper were taken at 0.90 (NOT98) and 0.84 (NOT98) orbital phases (see Fig. 18). In both spectra we observe the $\mathrm{H} \alpha, \mathrm{H} \beta$ and $\mathrm{Ca}$ II $\lambda 8662$ lines filled-in, and the $\mathrm{Ca}$ II $\mathrm{H} \& \mathrm{~K}$ lines in emission. 


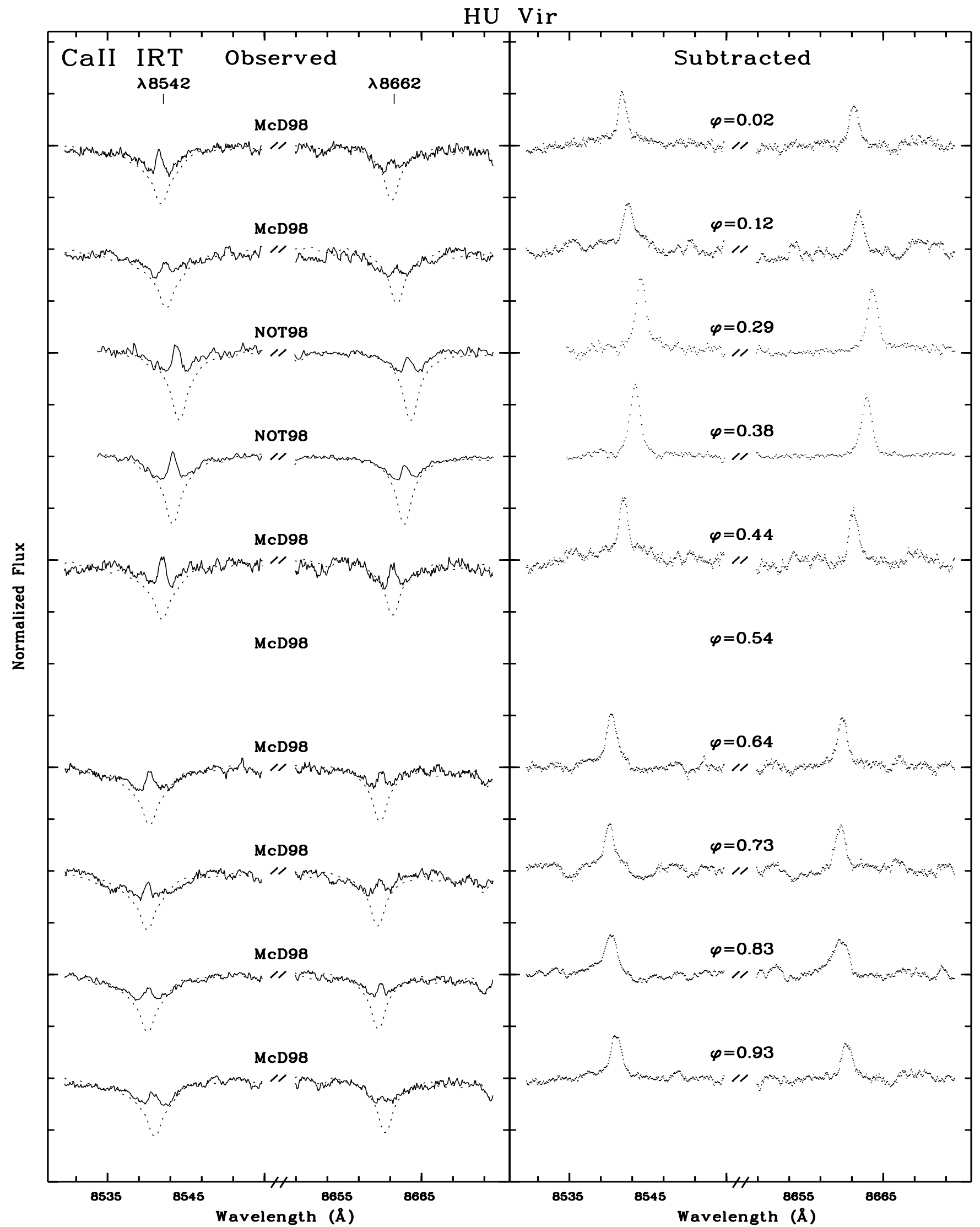

Fig. 17. Ca II IRT $\lambda 8542$ and $\lambda 8662$ observed (left panel) and subtracted (right panel) spectra of HU Vir 

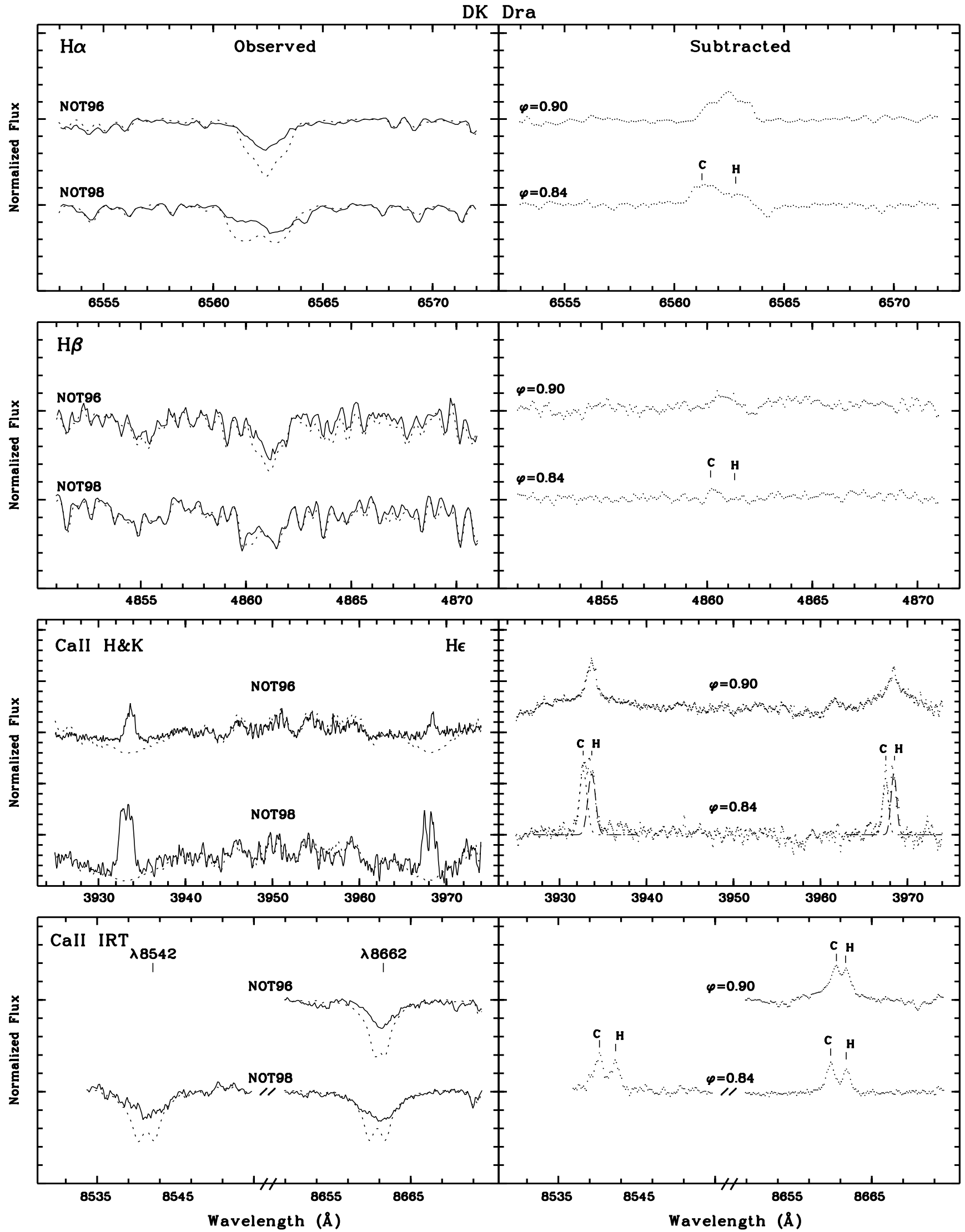

Fig. 18. $\mathrm{H} \alpha, \mathrm{H} \beta$, Ca II H \& K, and Ca II IRT spectra of DK Dra. Observed and synthetic spectra in the left panel and subtracted spectra in the right panel. We have also plotted the Gaussian fit to the subtracted spectrum used to deblend the contribution of the hot $(\mathrm{H})$ and cool $(\mathrm{C})$ components in the Ca II H \& K lines at 0.84 orbital phase (short- and long-dashed lines) 


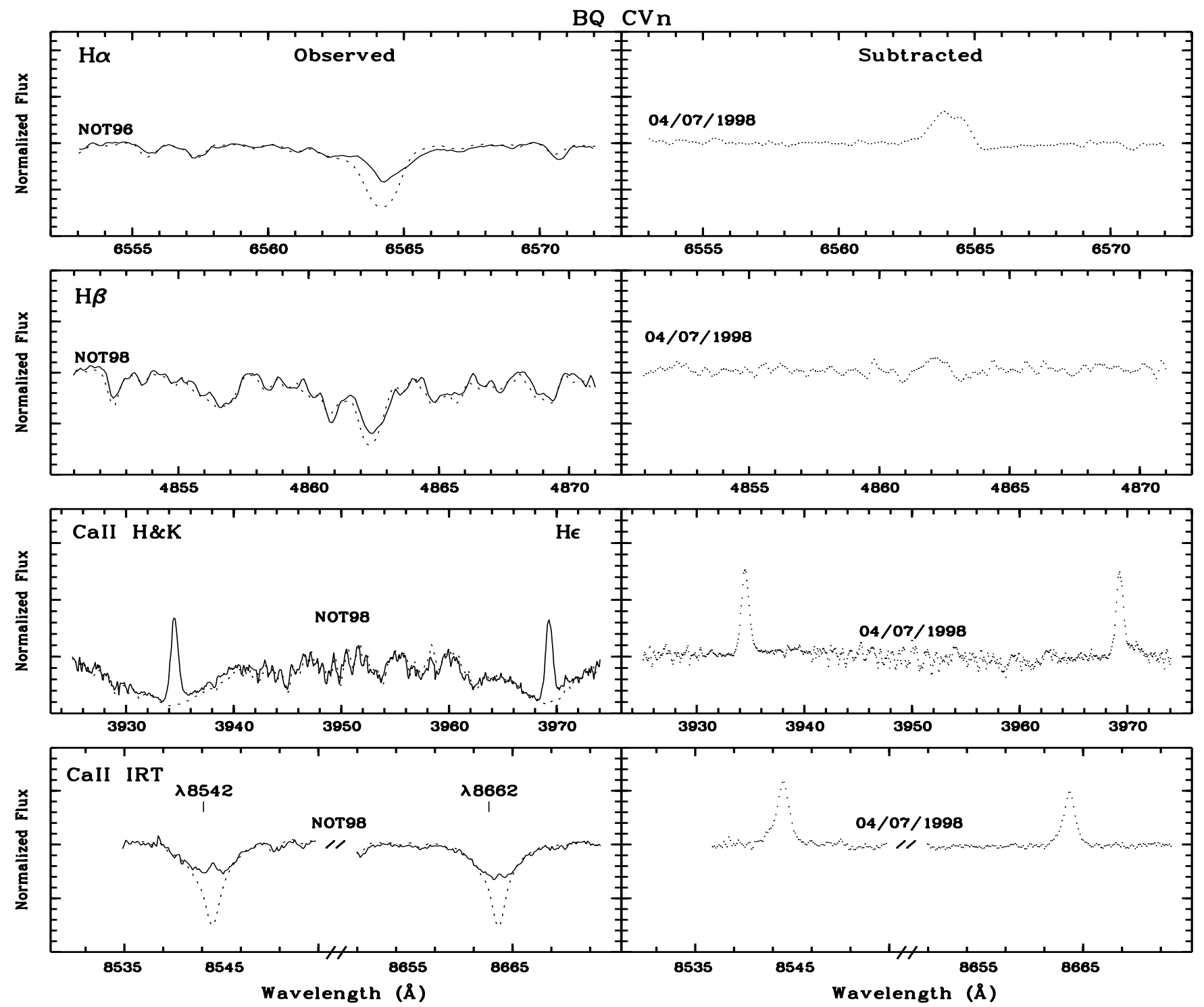

Fig. 19. $\mathrm{H} \alpha, \mathrm{H} \beta$, Ca II H \& K, and Ca II IRT spectra of BQ CVn. Observed and synthetic spectra in the left panel and subtracted spectra in the right panel

At 0.90 , we cannot separate the contribution of each component in $\mathrm{H} \alpha, \mathrm{H} \beta$ and $\mathrm{Ca}$ II $\mathrm{H} \& \mathrm{~K}$ lines, but due to the large wavelength of the Ca II $\lambda 8662$ line, a double peak is clearly observed in the subtracted spectrum of this line. In the 0.84 spectrum, we can see that the $\mathrm{H} \alpha$ line is filledin for both stars. Moreover, we can observe the excess $\mathrm{H} \alpha$ emission of the blue-shifted component is bigger than that of the red-shifted one. The excess $\mathrm{H} \beta$ emission seems to come only from the blue-shifted component. Although the $S / N$ ratio in the $\mathrm{Ca}$ II $\mathrm{H} \& \mathrm{~K}$ lines region is low, we can clearly see broad and two-piked emission in the $\mathrm{H} \& \mathrm{~K}$ lines. In the subtracted spectrum of the Ca II IRT $\lambda \lambda 8542$ and 8662 lines the emission from both components is clearly separated, being the emission of the blueshifted one slightly larger. All of this indicates that the blue-shifted component is slightly more active than the other component. Very small absorption is observed in the He I $\mathrm{D}_{3}$ line in the expected position for both components (see Fig. 24), contrary to the notable absorption observed in other giants of the sample. This is probably due to the blend with other photospheric lines of both stars in this SB2 system.

\subsection{2. $B Q C V n(H D$ 112859)}

A quarter of century ago Schild (1973) classified this star as a peculiar G8III-IV. Henry et al. (1995a) detected its Ca II H \& K emission and noticed that at red wavelengths the spectrum was double-lined although the intensity of the lines of both components was very different. They suggested a K0III primary and a late-F spectral type for the secondary. We have taken from these authors both the 

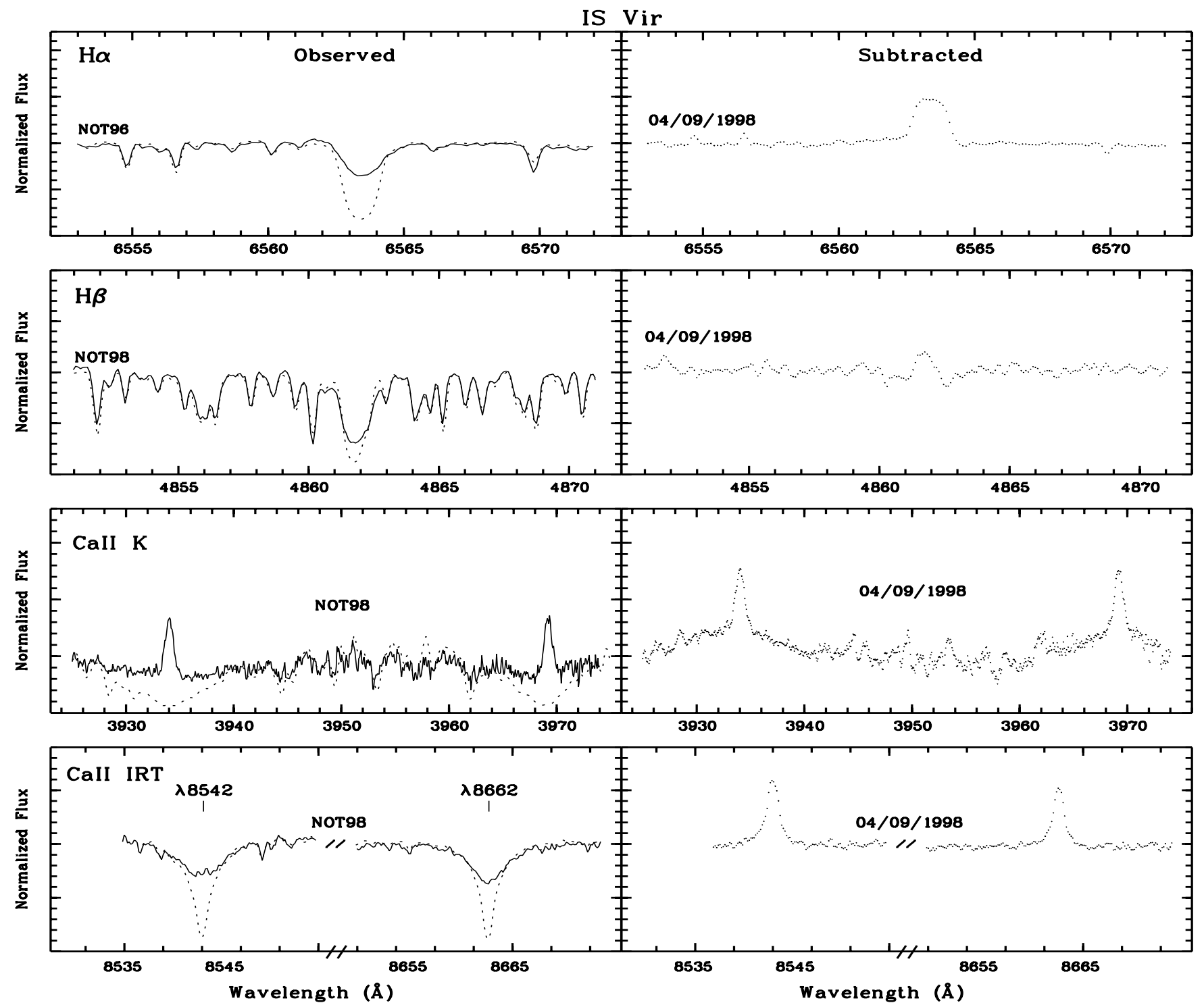

Fig. 20. $\mathrm{H} \alpha, \mathrm{H} \beta$, Ca II H \& K, and Ca II IRT spectra of IS Vir. Observed and synthetic spectra in the left panel and subtracted spectra in the right panel

orbital and rotational period and the radius. On the other hand, Strassmeier (1994b) confirmed the strong Ca II $\mathrm{H} \& \mathrm{~K}$ emission.

Orbital parameters have not been published, so we cannot calculate the orbital phase of our spectrum (NOT98). However, some conclusions can be obtained looking at the observed spectrum (Fig. 19): at infrared and red wavelengths, the secondary lines are not clear. A very weak blue-shifted absorption feature can be ascribed to the secondary in the $\mathrm{H} \alpha$ region. At shorter wavelengths, as in the $\mathrm{Na} \mathrm{I}_{1}, \mathrm{D}_{2}$ lines region, the spectral lines of the secondary are more conspicuous. In the $\mathrm{Ca}$ II $\mathrm{H} \& \mathrm{~K}$ lines region the contribution of the $\mathrm{F}$ star is evident in the broad Ca II absorption lines, where a clear red-shift of the emission can be seen. Taking into account what has been said above, we have calculated the synthesized spectrum using a F8V star as a template one for the secondary. In the observed spectrum, the $\mathrm{H} \alpha$ line shows a filling-in for the primary star. The $\mathrm{H} \beta$ line is slightly filled-in. The presence of the He I $\mathrm{D}_{3}$ absorption line is detected (Fig. 24). In our spectrum we observe strong $\mathrm{Ca}$ II $\mathrm{H} \& \mathrm{~K}$ emission and a weak $\mathrm{H} \epsilon$ emission line from the cool star. Finally, the Ca II IRT lines exhibit reversal emission.

\subsection{IS Vir (HD 113816)}

IS Vir is a single-lined spectroscopic binary classified as K0III by Henry et al. (1995a). We have taken from them the orbital and rotational periods and the radius given in Table 2. Strong Ca II H \& K emission was observed (Buckley et al. 1987; Strassmeier 1994b; Montes et al. 

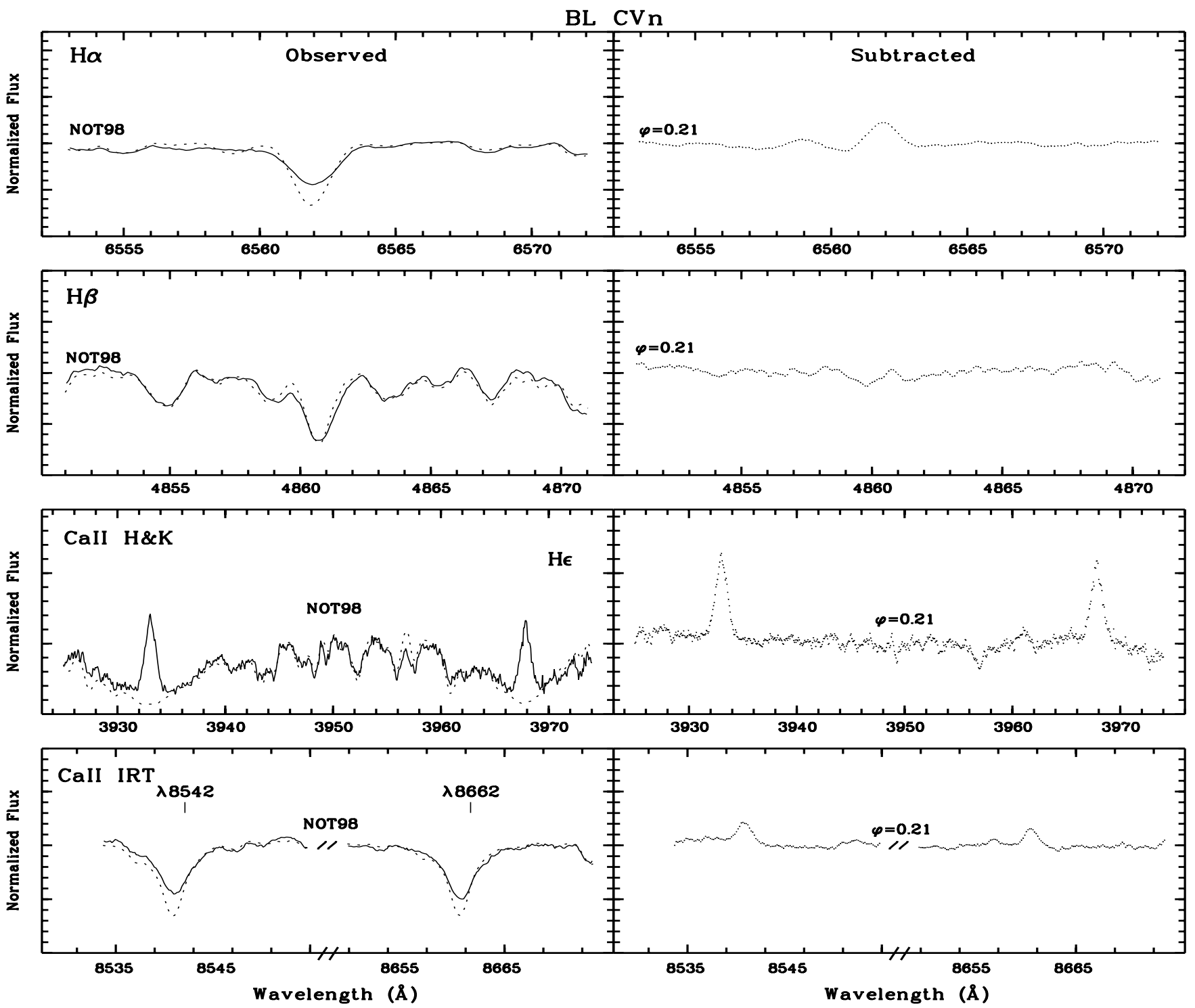

Fig. 21. $\mathrm{H} \alpha, \mathrm{H} \beta$, Ca II H \& K, and Ca II IRT spectra of BL CVn. Observed and synthetic spectra in the left panel and subtracted spectra in the right panel

1995c). In our previous observation of this system in the Ca II H \& K lines region in Mar-93 at 0.68 orbital phase (Montes et al. 1995c) we found strong emission in the $\mathrm{H}$ \& $\mathrm{K}$ lines with intensity above the continuum at $3950 \AA$ A, but lower than reported by Strassmeier (1994b).

In the new spectrum (NOT 98) (Fig. 20), the H $\alpha$ line and the Ca II IRT lines show intense filled-in absorption, whereas the $\mathrm{H} \beta$ line only shows a slight filling-in. In the $\mathrm{Ca}$ II $\mathrm{H} \& \mathrm{~K}$ lines region the $S / N$ ratio is low and the synthetic and observed spectra are not well matched, but a strong emission in the $\mathrm{H} \& \mathrm{~K}$ lines well above the continuum is observed. The He $\mathrm{I}_{3}$ line appears in absorption (Fig. 24).

\subsection{BL CVn (HD 115781)}

This double-lined spectroscopy binary was classified by Hall (1990) as K1III + FIV, and later as K1III + G5IV by Stawikowski \& Glebocki (1994). Lines et al. (1985) found this system to have a photometric period of $9.31 \pm$ 0.06 days and an amplitude of $0.16 \mathrm{mag}$. They attributed the light variability to the ellipticity effect because the orbital period was twice the photometric period and times of maximum brightness occurred at times of maximum positive radial velocity. It is a nearly-synchronous binary: its orbital period is $18.6917 \pm 0.0011$ days (Griffin \& Fekel 1988 ) and its rotational period is 18.70 days (Stawikowski \& Glebocki 1994). The orbital eccentricity cannot be far from zero (Griffin \& Fekel 1988). Fekel et al. (1986) found $V \sin i$ values of $(35 \pm 2) \mathrm{km}^{-1}$ and $(7 \pm 2) \mathrm{km}^{-1}$ for the primary and secondary, respectively. The great line 

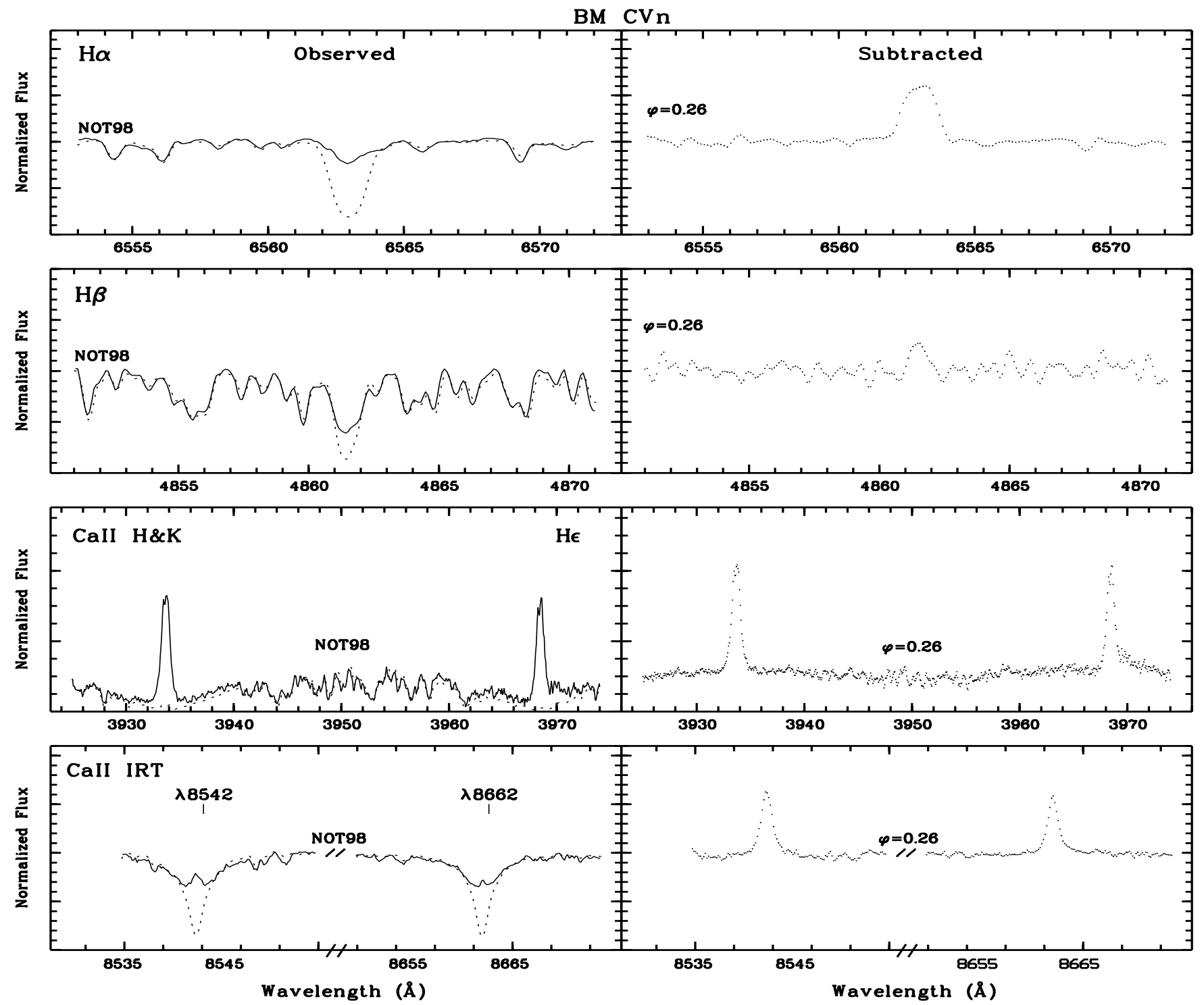

Fig. 22. $\mathrm{H} \alpha, \mathrm{H} \beta$, Ca II H \& K, and Ca II IRT spectra of BM CVn. Observed and synthetic spectra in the left panel and subtracted spectra in the right panel

broadening of BL CVn and the ellipsoidal light variations might suggest that the $\mathrm{K}$ giant star is close to filling its Roche lobe (Griffin \& Fekel 1988). Moderate H $\alpha$ absorption is found by Fekel et al. (1986). Strassmeier et al. (1990) found strong Ca II H \& K emission.

In our present observations (NOT98, Fig. 21) the $\mathrm{H} \alpha$ and $\mathrm{H} \beta$ lines appears in absorption in the observed spectrum. After applying the spectral subtraction technique, we only obtain small excess $\mathrm{H} \alpha$ emission. Broad emission is observed in the $\mathrm{Ca}$ II $\mathrm{H} \& \mathrm{~K}$ lines. We observe small absorption in the $\mathrm{He} \mathrm{I}_{3}$ line (Fig. 24). A small filling-in is obtained in the Ca II $\lambda 8542$ and $\lambda 8662$ lines.

\subsection{5. $B M C V n(H D 116204)$}

This single-lined spectroscopy binary was classified by Keenan (1940) as K1III. It is a nearly-synchronous binary: its orbital period is $(20.6252 \pm 0.0018)$ days (Griffin $\&$ Fekel 1988) and its rotational period is (20.66 \pm 0.03$)$ days (Strassmeier et al. 1989a). The orbit is judged to be circular (Griffin \& Fekel 1988). It is also a relatively fast rotator, Fekel et al. (1986) found rotationally broadened lines with $V \sin i=(15 \pm 2) \mathrm{km}^{-1}$. This system shows strong Ca II H \& K emission (Bidelman 1983), together with an emission-line spectrum typical of RS CVn stars in the IUE ultraviolet region, but $\mathrm{H} \alpha$ is an absorption line (Griffin \& Fekel 1988).

In our present spectrum (NOT98, Fig. 22) we observe the $\mathrm{H} \alpha$ line as nearly total filled-in absorption. After applying the spectral subtraction technique, strong excess 

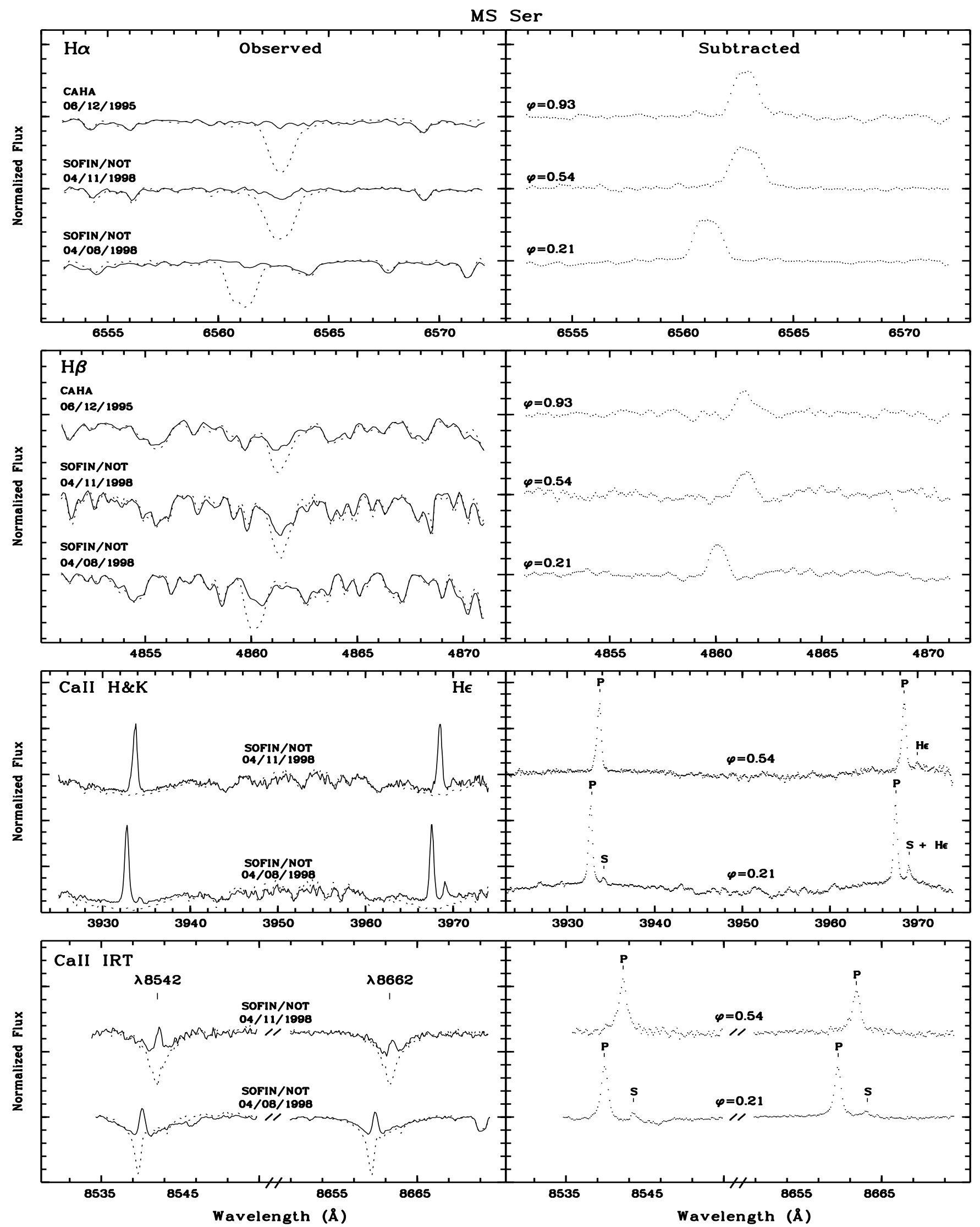

Fig. 23. $\mathrm{H} \alpha, \mathrm{H} \beta$, Ca II H \& K, and Ca II IRT spectra of MS Ser. Observed and synthetic spectra in the left panel and subtracted spectra in the right panel 
$\mathrm{H} \alpha$ emission is obtained. The $\mathrm{H} \beta$ line appears as an absorption line in the observed spectrum and clear excess emission is observed in the subtracted spectrum. The $\frac{E_{\mathrm{H} \alpha}}{E_{\mathrm{H} \beta}}$ ratio obtained is larger than 3 , which indicates that the emission would arise from extended regions viewed off the limb. The Ca II H \& K lines show strong emission, with a blue-shifted self-absorption feature, and small emission is also detected in the $\mathrm{H} \epsilon$ line. The $\mathrm{He} \mathrm{I}_{3}$ line appears in absorption (Fig. 24). A clear emission reversal is observed in the Ca II IRT $\lambda 8542$ and $\lambda 8662$ lines.

\subsection{6. $M S \operatorname{Ser}(H D 143313)$}

Griffin (1978) first observed the binary nature of MS Ser calculating the orbital elements for this system (see Table 2). Griffin gave its $T_{0}$ in MJD, and this yielded Strassmeier et al. (1993) to a bad calculation of the $T_{0}$ in HJD. Griffin also proposed $\mathrm{K} 2 \mathrm{~V} / \mathrm{K} 6 \mathrm{~V}$ as spectral types of the components, based on photometric arguments for the secondary star. Bopp et al. (1981b) observed a variable filling-in of the $\mathrm{H} \alpha$ line and calculated a photometric period of 9.60 days, slightly different from the orbital period. Miller \& Osborn (1996) confirmed the value of the photometric period and Strassmeier et al. (1990) observed strong emission in the Ca II H \& K composite spectrum. Dempsey et al. (1993a) noted some filling-in in the Ca IRT lines for MS Ser, but not reverse emission. Alekseev (1999) made a photometric and polarimetric study of MS Ser, calculating a spot area of $15 \%$ of the total stellar surface, and observed some seasonal variations. Finally, Osten \& Saar (1998) revised the stellar parameters for MS Ser and suggested K2IV/G8V as a better classification. We have also found that the primary component may have a luminosity class IV or higher based on our analysis of some metallic lines, like the Ti I lines, the Hipparcos data and the Wilson Bappu effect (see Sanz-Forcada et al. 1999).

Two spectra of MS Ser were taken in the NOT98 observing run. Moreover, we analyse here another spectrum taken on 12th June 1995 with the $2.2 \mathrm{~m}$ telescope at the German Spanish Observatory (CAHA) in Calar Alto (Almería, Spain), using a Coudé spectrograph with the $\mathrm{f} / 3$ camera, CCD RCA \#11 covering two ranges: $\mathrm{H} \alpha$ (from 6510 to $6638 \AA$ ) and $\mathrm{H} \beta$ (from 4807 to $4926 \AA$ ), with a resolution of $\Delta \lambda 0.26$ in both cases.

The $H \alpha$ and $H \beta$ lines: In both lines, we observe a nearly total filling-in in the 1995 spectra and small absorption in the spectra taken in the NOT98 observing run. After applying the spectral subtraction, a clear filling-in in the $\mathrm{H} \alpha$ and $\mathrm{H} \beta$ lines is observed in the three spectra (see Fig. 23). The $\mathrm{H} \alpha$ line of this system is highly variable. We have found, in the present spectra, a variable filling-in whereas Bopp et al. (1981b) found $\mathrm{H} \alpha$ was a weak emission line. In the three new spectra we have taken of this system with the FOCES echelle spectrograph in July 1999 (forthcoming paper) we observe variable $\mathrm{H} \alpha$ emission well above the continuum.

The $C a$ II $H$ \& $K$ and $H \epsilon$ lines: Strong emission in these lines and the $\mathrm{H} \epsilon$ line in emission arising from the primary component was observed in our previous observation of this system in March 1993 at 0.16 orbital phase (Montes et al. 1995c). In the present observations (Fig. 23) we have deblended the emission arising from both components in the spectrum taken near to the quadratures $(\varphi=$ $0.21)$. The strongest emission, centered at the absorption line, arises from the K2IV component, which is the component with larger contribution to the continuum. The red-shifted and less intense emission corresponds to the G8V secondary component. In the $\varphi=0.55$ observation we cannot separate the contribution from both stars. The $\mathrm{H} \epsilon$ line appears in emission in both spectra. The emission intensity observed in our 1993 and 1998 spectra is larger than the emission intensity observed in the 1988 spectrum presented by Strassmeier et al. (1990).

The Ca II IRT lines: A clear emission reversal is observed in the core of the Ca II IRT absorption lines $\lambda 8542$ and $\lambda 8662$ in both spectra (Fig. 23). After applying the spectral subtraction technique, we can see small excess emission arising from the secondary component, in the 0.21 spectrum, as in the case of the Ca II H \& K lines. This emission reversal observed here clearly contrasts with the only filling-in in these lines reported by Dempsey et al. (1993a).

The $\mathrm{He}$ I $\mathrm{D}_{3}$ line: We can distinguish (Fig. 24) the $\mathrm{He} \mathrm{I}_{3}$ line as a very small absorption from the primary star. A slight variation is observed between the spectra at 0.21 and 0.55 orbital phases. The luminosity class of this star (IV-III) and the SB2 nature of the system could be the reason of this small absorption in the $\mathrm{He} \mathrm{I}_{3} \mathrm{D}_{3}$ line in relation with that observed in other giants of the sample.

\section{Discussion and conclusions}

In this paper we have analysed, using the spectral subtraction technique, high resolution echelle spectra of 16 chromospherically active binary systems. These spectra include all the optical chromospheric activity indicators from the $\mathrm{Ca}$ II $\mathrm{H} \& \mathrm{~K}$ to $\mathrm{Ca}$ II IRT lines, and in some observing runs the $\mathrm{Li}$ I $\lambda 6707.8$ line too.

$\mathrm{H} \alpha$ emission above the continuum is observed in UX Ari and HU Vir, in the rest of the systems excess $\mathrm{H} \alpha$ emission is clearly detected in the subtracted spectra. Filled-in absorption $\mathrm{H} \beta$ line profiles have been found in all the stars of the sample except LR Hya and BL CVn, which also have a lower level of activity in the other chromospheric activity indicators.

Very broad and variable extended wings have been found in the subtracted $\mathrm{H} \alpha$ profile of the very active star HU Vir. These line profiles are not well matched using a single-Gaussian fit, and have been fitted using 


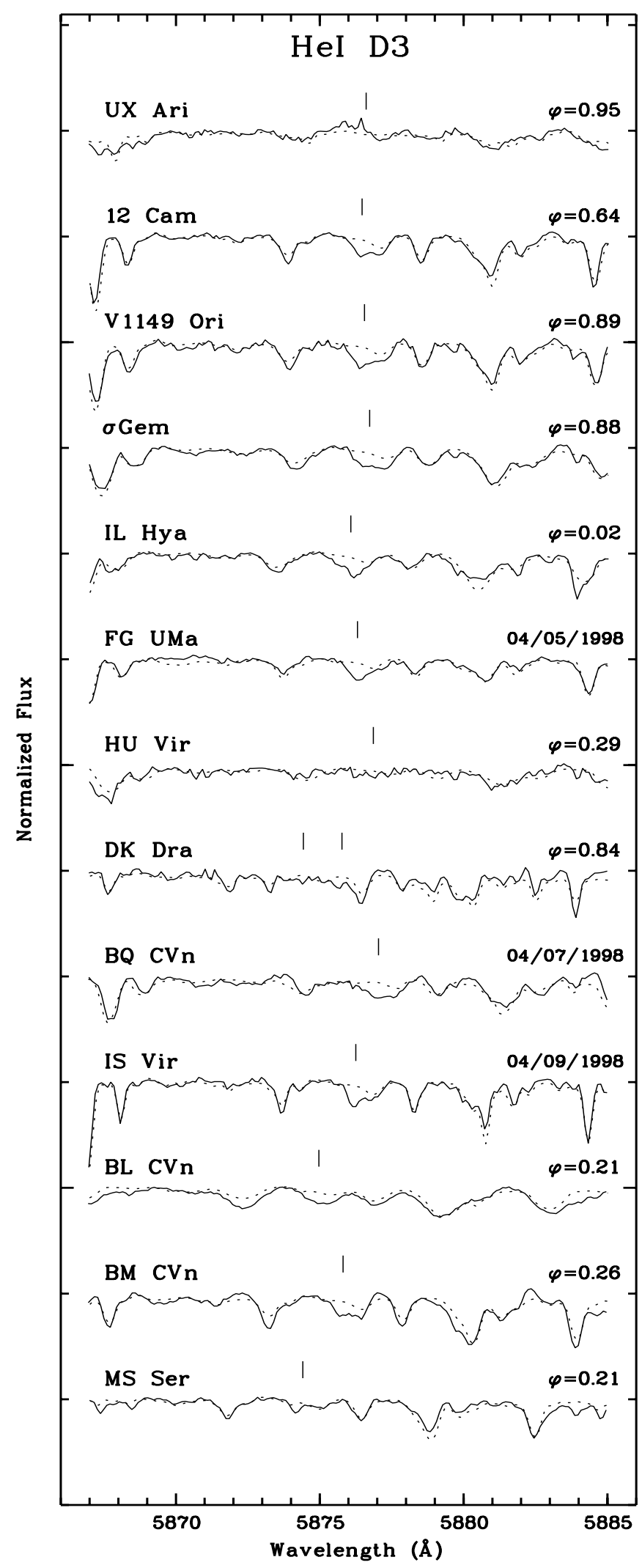

Fig. 24. He $\mathrm{I}_{3}$ line for selected stars of the sample. We plot observed spectrum (solid-line) and the synthesized spectrum (dashed-line). The expected position of the $\mathrm{He} \mathrm{I}_{3}$ line for each star is marked with a short vertical line two Gaussian components (narrow and broad). Similar behaviour has been found in other very active stars (Hatzes 1995; Jones et al. 1996; Stauffer et al. 1997; Papers I, II, Montes et al. 1998b). We have interpreted this broad component emission as arising from microflaring (high turbulence emitting plasma) activity that takes place in the chromosphere by similarity with the broad components found in the chromospheric $\mathrm{Mg}$ II h \& k lines (Wood et al. 1996; Busà et al. 1999) and in several transition region lines of active stars observed with GHRS-HST (Linsky \& Wood 1994; Linsky et al. 1995; Wood et al. 1996, 1997; Dempsey et al. 1996a,b; Robinson et al. 1996) and recently confirmed with STIS-HST observations (Pagano et al. 2000). In some cases the $\mathrm{H} \alpha$ line is asymmetric and the fit is better matched when the broad component is blue-shifted or red-shifted with respect to the narrow component. These asymmetries are also observed during the impulsive and gradual phases of solar and stellar flares (Montes et al. 1996b, 1999; Montes \& Ramsey 1999), and favour the interpretation of the broad component as due to upward and downward motions produced by microflaring in the chromosphere.

Excess absorption and emission is observed in the wings of the $\mathrm{H} \alpha$ and $\mathrm{H} \beta$ lines of several systems. Absorption features are detected by us in the blue wing of V1149 Ori and in the red wing of 12 Cam (also observed by Eker et al. 1995), IL Hya (also detected by Weber \& Strassmeier 1998) and HU Vir (also seen by Strassmeier 1994a, and Hatzes 1998). We detected excess emission in the blue wing of FG UMa too. Similar red-shifted absorption features were already seen by other authors in the single star OP And (Fekel et al. 1986) and in the binaries VY Ari (Bopp et al. 1989); XX Tri (Bopp et al. 1993) and IN Vir (Strassmeier 1997). Blue-shifted emission was also detected in the single giant YY Men (Vilhu et al. 1991). Several dynamical processes, or a combination of them, could be the origin of these blue-shifted or red-shifted absorption features: plage- and prominence-like structures (Hall \& Ramsey 1992, 1994; Neff 1996; Eibe 1998); continuous low-level mass infall (Walter \& Byrne 1998; Walter 1999; Eibe et al. 1999); local velocity fields and mass motions due to magnetic field inhomogeneities possibly coupled with a loop-like geometry (Strassmeier 1994a); fluctuations of both the column density and temperature gradient within the chromosphere (Smith \& Dupree 1988); stellar winds and anti-winds (Linsky et al. 1995).

Prominence-like extended material viewed off the limb has been detected in many stars of the sample according to the high ratios $E_{\mathrm{H} \alpha} / E_{\mathrm{H} \beta}$ obtained. Prominences viewed against the disk seem to be present at some orbital phases in the dwarfs OU Gem and BF Lyn.

The application of the spectral subtraction reveals that the $\mathrm{He}$ I $\mathrm{D}_{3}$ line appears as an absorption feature (Fig. 24) in mainly all the giants of the sample as in the case of the stars analysed in Paper I. Total filling-in of $\mathrm{He} \mathrm{I}_{3}$ is 
observed in the very active star HU Vir, similar to the behaviour observed in EZ Peg (Paper II). These results are in agreement with the behaviour reported by Saar et al. (1997). These authors found that while for few active stars the He I $\mathrm{D}_{3}$ line behaves "normally", increasing in absorption with increasing rotation, and showing consistent correlations with other activity indicators, the behaviour clearly diverges (large filling-in) when stars become very active, suggesting that the line could be filled-in due to frequent low-level flaring. In the most evolved stars the behaviour is different as a consequence of the lower chromospheric densities of these stars.

Ca II $\mathrm{H} \& \mathrm{~K}$ emission is observed in all the stars of the sample. Small emission in the close $\mathrm{H} \epsilon$ line is also detected in some of the more active stars. In some systems like OU Gem, BF Lyn, LR Hya, DK Dra and MS Ser the emission from both components is clearly deblended in these lines. Self-reversed absorption core with red asymmetry $\left(I\left(\mathrm{~K}_{2 \mathrm{~V}}\right)<I\left(\mathrm{~K}_{2 \mathrm{R}}\right)\right)$ is detected in the Ca II $\mathrm{H} \& \mathrm{~K}$ lines of the giants 12 Cam, FG UMa and BM CVn. The self-reversed feature is a consequence of the line formation process in the chromosphere (depth variation of the line source function in an atmosphere having a chromospheric temperature rise). Asymmetries in these profiles provide information on velocity fields in the line formation regions. In these three giants we observed a small red asymmetry (indicative of outward mass flux, wind), contrary to the blue asymmetry (indicative of upward propagating waves, but not large wind) normally observed in giant stars hotter than spectral type K3 (Stencel 1978) and also observed by us (FFMCC) in the giants (V1817 Cyg and V1764 Cyg).

The Ca II IRT lines result to be a very useful chromospheric activity indicator too. We have found that all the stars analysed here show a clear filled-in absorption line profile or even notable emission reversal (UX Ari, OU Gem, BF Lyn, IL Hya, HU Vir, BQ CVn, BM CVn, MS Ser). Thanks to the higher resolution of our spectra, we were able to detect emission reversal in the Ca II IRT lines in some systems in which previous studies (Linsky et al. 1979; Dempsey et al. 1993a) only reported a filling-in. An increase in the level of activity of these stars could also be the cause of these detections. When both components of the binary system are active the excess emission detected in the Ca II IRT lines is much more easily deblended thanks to the large wavelength of these lines (see e.g. DK Dra, Fig. 18). We have found $E_{8542} / E_{8498}$ ratios in the range $\simeq 1-2$ which is indicative of optically thick emission in plage-like regions, in contrast with the prominencelike material inferred by the $E_{\mathrm{H} \alpha} / E_{\mathrm{H} \beta}$ ratios. These small $E_{8542} / E_{8498}$ ratios, also found by Chester et al. (1994) and Arévalo \& Lázaro (1999) in other active binaries, indicate the existence of distinct sources of Balmer and Ca II IRT emission and suggest that the activity of these very active stars is not simply a scaled-up version of solar activity.

Some systems have been observed at different orbital phases and different epochs, covering all the orbital cycle

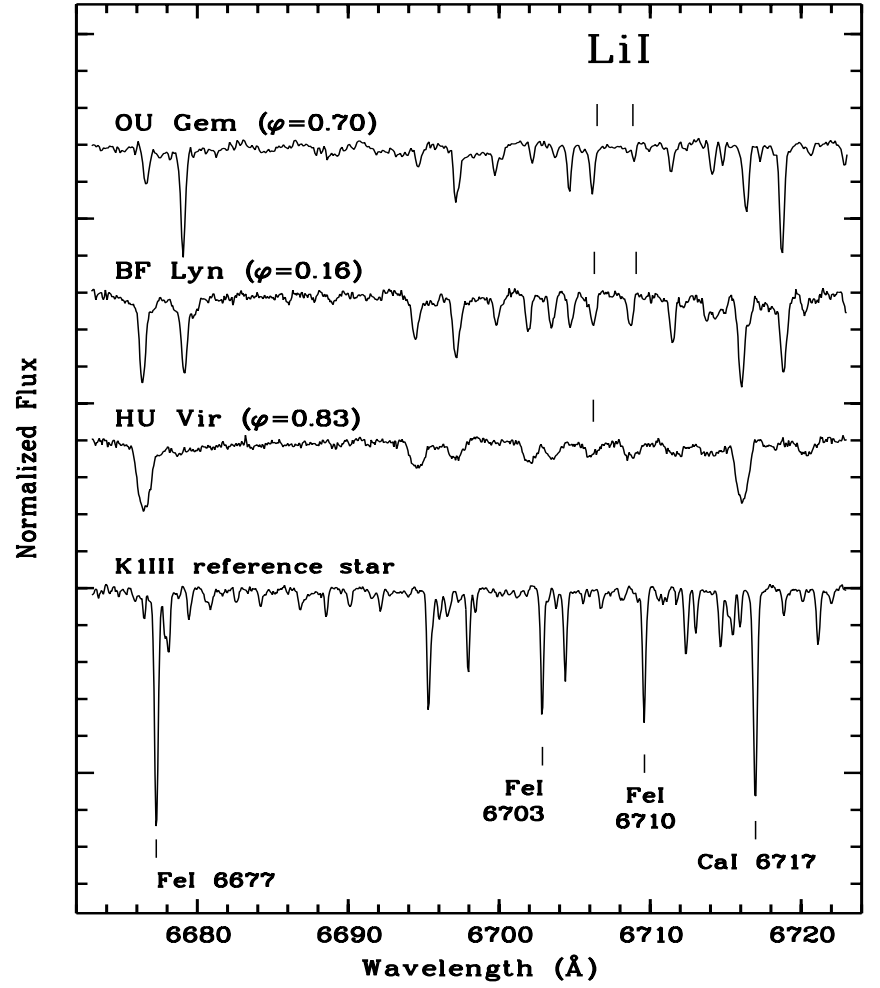

Fig. 25. Spectra of OU Gem, BF Lyn and HU Vir in the Li I $\lambda 6707.8$ line region. The expected position of the $\mathrm{Li}$ I line for each star is marked with a short vertical line. We have also plotted a K1III reference star with some photospheric lines identified

and it was possible to study the variability of the chromospheric emission. The excess $\mathrm{H} \alpha$ and Ca II IRT emission of BF Lyn in the McD98 observing run shows anti-correlated variations with the orbital phase (see Fig. 8). This anticorrelation could indicate that the chromospheric active regions are concentrated on faced hemispheres of both components but at about 0.4 and 0.9 orbital phases for the hot and cool component, respectively. Evidence of an active longitude area $(0.29-0.44$ orbital phase interval $)$ has been found in HU Vir when we compare with the higher level of activity in this phase interval also reported by Strassmeier (1994a), in 1991, and Hatzes (1998), in 1995.

Acknowledgements. We would like to thank Dr. L.W. Ramsey for collaborating in the McDonald observing run and the staff of McDonald observatory for their allocation of observing time and their assistance with our observations. We would like to thank Dr. B.H. Foing for allow us to use the ESA-MUSICOS spectrograph at Isaac Newton Telescope. We would also like to thank the referee S. Catalano for suggesting several improvements and clarifications. This work has been supported by the Universidad Complutense de Madrid and the Spanish Dirección General de Enseñanza Superior e Investigación Científica (DGESIC) under grant PB97-0259. 


\section{References}

Alekseev I.Y., 2000, ARep 44, 178

Arévalo M.J., Lázaro C., 1999, AJ 118, 1015

Barden S.C., 1985, ApJ 295, 162

Barden S.C., Nations H.L., 1985, in Cool Stars, Stellar Systems, and the Sun, Zeilik M., Gibson D.M. (eds.). Springer, p. 262

Barrado D., Fernández-Figueroa M.J., García López R.J., De Castro E., Cornide M., 1997, A\&A 326, 780

Barrado D., De Castro E., Fernández-Figueroa M.J., Cornide M., García López R.J., 1998, A\&A 337, 739

Baudrand J., Böhm T., 1992, A\&A 259, 711

Berdyugina S.V., Tuominen I., 1998, A\&A 336, L25

Berdyugina S.V., Ilyin I., Tuominen I., 1999, A\&A 349, 863

Bidelman W.H., 1964, ApJ 139, 405

Bidelman W.P., 1981, AJ 86, 553

Bidelman W.P., 1983, AJ 88, 1182

Bidelman W.P., McConnell D.J., 1973, AJ 78, 687

Bopp B.W., 1980, PASP 92, 218

Bopp B.W., 1983, in: IAU Colloq. 71, Activity in Red-Dwarf Stars, Byrne P.B., Rodonò M. (eds.). Dordrecht: Reidel, p. 363

Bopp B.W., Talcott J.C., 1978, AJ 83, 1517

Bopp B.W., Fekel F.C., Griffin R.F., Beavers W.I., Gunn J.E., Edwards D., 1979, AJ 84, 1763

Bopp B.W., Hall D.S., Henry G.W., Noah P., Klimke A., 1981a, PASP 93, 504

Bopp B.W., Noah P., Klimke A., Africano J.L., 1981b, ApJ 249, 210

Bopp B.W., Africano J.L., Goodrich B.D., Henry G.W., Hall D.S., Barksdale W.S. Jr., 1984, IBVS 2604, 1

Bopp B.W., Saar S.H., Ambruster C., et al., 1989, ApJ 339, 1059

Bopp B.W., Fekel F.C., Aufdenberg J.P., Dempsey R.C., Dadonas V., 1993, AJ 106, 2502

Buckley D.A., Tuohy I.R., Remillard R.A., Bradt H.V., Schwartz D.A., 1987, ApJ 315, 273

Busà I., Pagano I., Rodonò M., Neff J.E., Lanzafame A.C., 1999, A\&A 350, 571

Buzasi D.L., 1989, Ph.D. Thesis, Pennsylvania State Univ.

Carlos R.C., Popper D.M., 1971, PASP 83, 504

Cutispoto G., 1991, A\&AS 89, 435

Cutispoto G., 1993, A\&AS 102, 655

Cutispoto G., 1995, A\&AS 111, 507

Chester M.M., 1991, Ph.D. Thesis, Pennsylvania State Univ.

Chester M.M., Hall J.C., Buzasi D.L., 1994, in ASP Conf. Ser. 64, 390. The Eighth Cambridge Workshop on Cool Stars, Stellar Systems, and the Sun, Caillault J.-P. (ed.), San Francisco: ASP

Dempsey R.C., Bopp B.W., Henry G.W., Hall D.S., 1993a, ApJS 86, 293

Dempsey R.C., Linsky J.L., Fleming T.A., Schmitt J.H.M.M., 1993b, ApJ 413, 333

Dempsey R.C., Neff J., O'Neal D., Olah K., 1996a, AJ 111, 1356

Dempsey R.C., Neff J., Linsky J.L., Brown A., 1996b, in IAU Symp. 176, Stellar Surface Structure, Strassmeier K.G., Linsky J.L. (eds.). Kluwer Acad. Pub., p. 411

Dempsey R.C., Linsky J.L., Fleming T.A., Schmitt J.H.M.M., 1997, ApJ 478, 358
Donati J.-F., Semel M., Carter B.D., Rees D.E., Cameron A.C., 1997, MNRAS 291, 658

Duemmler R., Aarum V., 2000, in: ASP Conf. Ser., The 11th Cambridge Workshop on Cool Stars, Stellar Systems, and the Sun, García López R., Rebolo R., Zapatero Osorio M.R. (eds.) (in press)

Duemmler R., Ilyin I., Tuominen I., 1997, A\&AS 123, 209

Eibe M.T., 1998, A\&A 337, 757

Eibe M.T., Byrne P.B., Jeffries R.D., Gunn A.G., 1999, A\&A 341,527

Eker Z., Hall D.S., Anderson C.M., 1995, ApJS 96, 581

ESA, 1997, The Hipparcos and Tycho Catalogues, ESA SP-1200

Fekel F.C., 1997, PASP 109, 514

Fekel F.C., Moffett T.J., Henry G.W., 1986, ApJS 60, 551

Fekel F.C., Gillies K., Africano J., Quigley R., 1988, AJ 96, 1426

Fekel F.C., Strassmeier K.G., Weber M., Washüttl A., 1999, A\&AS 137, 369

Fernández-Figueroa M.J., Montes D., De Castro E., Cornide M., 1994, ApJS 90, 433 (FFMCC)

Frasca A., Catalano S., 1994, A\&A 284, 883

Griffin R.F., 1978, Obs. 98, 257

Griffin R.F., Emerson B., 1975, Observatory 95, 23

Griffin R.F., Fekel F.C., 1988, JApA 9, 213

Gunn A.G., Doyle J.G., 1997, A\&A 318, 60

Gunn A.G., Doyle J.G., Houdebine E.R., 1997, A\&A 319, 211

Hall D.S., 1990, AJ 100, 554

Hall J.C., 1996, PASP 108, 313

Hall J.C., Ramsey L.W., 1992, AJ 104, 1942

Hall J.C., Ramsey L.W., 1994, AJ 107, 1149

Hall J.C., Wolovitz J.B., 1998, AJ 115, 2571

Hall D.S., Fekel F.C., Henry G.W., Eaton J.A., et al., 1995, AJ 109, 1277

Hatzes A.P., 1995, AJ 109, 350

Hatzes A.P., 1998, A\&A 330, 541

Henry G.W., Fekel F.C., Hall D., 1995a, AJ 110, 2926

Henry G.W., Eaton J.A., Hamer J., Hall D.S., 1995b, ApJS 97,513

Hirshfeld A., Sinnott R.W., 1982, Sky Catalogue 2000, Vol. 1

Huenemoerder D.P., Buzasi D.L., Ramsey L.W., 1989, AJ 98, 2264

Jetsu L., 1996, A\&A 314, 153

Jones B.F., Fischer D.A., Stauffer J.R., 1996, AJ 112, 1562

Keenan P.C., 1940, ApJ 91, 506

Latorre A., Montes D., Fernández-Figueroa M.J., 1999, in 12th EADN Predoctoral School, "Selected Topics on Binary Stars", Observations and Physical Processes

Latorre A., Montes D., Fernández-Figueroa M.J., 2000, in: ASP Conf. Ser., The 11th Cambridge Workshop on Cool Stars, Stellar Systems, and the Sun, García López R., Rebolo R., Zapatero Osorio M.R. (eds.) (in press)

Lázaro C., Arévalo M.J., 1997, AJ 113, 2283

Lines R.D., Barksdale W.S., Stelzer H.J., Hall D.S., 1985, IBVS 2728

Linsky J.L., Hunten D., Glacken D., Kelch W., 1979, ApJS 41, 481

Linsky J.L., Wood B.E., 1994, ApJ 430, 342

Linsky J.L., Wood B.E., Judge P., Brown A., Andrulis C., Ayres T.R., 1995, ApJ 442, 381

McCarthy J.K., Sandiford B.A., Boyd D., Booth J., 1993, PASP 105, 881 
Miller R., Osborn W., 1996, Obs. 116, 382

Mitrou C.K., Doyle J.G., Mathioudakis K., Antonopoulou E., 1996, A\&AS 115, 61

Montes D., 1995, Ph.D. Thesis, Universidad Complutense de Madrid

Montes D., 1998, Ap\&SS 263, 275 (http://www.ucm.es/info/Astrof/spectra.html)

Montes D., Ramsey L.W., 1998, A\&A 340, L5

Montes D., Ramsey L.W., 1999, in: Solar and Stellar Activity: Similarities and Differences, Butler C.J., Doyle J.G. (eds.), ASP Conf. Ser. 158, 226

Montes D., Fernández-Figueroa M.J., De Castro E., Cornide M., 1994, A\&A 285, 609

Montes D., Fernández-Figueroa M.J., De Castro E., Cornide M., 1995a, A\&A 294, 165

Montes D., Fernández-Figueroa M.J., De Castro E., Cornide M., 1995b, A\&AS 109, 135

Montes D., De Castro E., Fernández-Figueroa M.J., Cornide M., 1995c, A\&AS 114, 287

Montes D., Fernández-Figueroa M.J., Cornide M., De Castro E., 1996a, A\&A 312, 221

Montes D., Sanz-Forcada J., Fernández-Figueroa M.J., Lorente R., 1996b, A\&A 310, L29

Montes D., Fernández-Figueroa M.J., De Castro E., SanzForcada J., 1997, A\&AS 125, 263 (Paper I)

Montes D., Sanz-Forcada J., Fernández-Figueroa M.J., De Castro E., Poncet A., 1998a, A\&A 330, 155a (Paper II)

Montes D., Fernández-Figueroa M.J., De Castro E., Cornide M., Poncet A., Sanz-Forcada J., 1998b, in ASP Conf. Ser. 154, CD-1516, The Tenth Cambridge Workshop on Cool Stars, Stellar Systems, and the Sun, Donahue R.A., Bookbinder J.A. (eds.), San Francisco: ASP

Montes D., Sanz-Forcada J., Fernández-Figueroa M.J., De Castro E., Cornide M., Ramsey L.W., 1998c, Ap\&SS 263, 279

Montes D., Saar S.H., Collier Cameron A., Unruh Y.C., 1999, MNRAS 305, 45

Montes D., Latorre A., Fernández-Figueroa M.J., 2000a, Stellar clusters and associations: convection, rotation, and dynamos, Pallavicini R., Micela G., Sciortino S. (eds.), ASP Conf. Ser. 198, 203

Montes D., Lachaume R., Fernández-Figueroa M.J., 2000b, in: ASP Conf. Ser., The 11th Cambridge Workshop on Cool Stars, Stellar Systems, and the Sun, García López R., Rebolo R., Zapatero Osorio M.R. (eds.) (in press)

Montes D., Fernández-Figueroa M.J., De Castro E., Cornide M., Latorre A., 2000c, in: ASP Conf. Ser., The 11th Cambridge Workshop on Cool Stars, Stellar Systems, and the Sun, García López R., Rebolo R., Zapatero Osorio M.R. (eds.) (in press)

Neff J.E., 1996, in IAU Symp. 176, Stellar Surface Structure, Strassmeier K.G., Linsky J.L. (eds.). Kluwer Acad. Pub., p. 113

Oláh K., Hall D.S., Henry G.W., 1991, A\&A 251, 531

Oliveira J.M., Foing B.H., 1999, A\&A 343, 213

Osten R.A., Saar S.H., 1998, MNRAS 295, 257

Pagano I., Linsky J.L., Robinson R.D., Carkner L., Woodgate B., 2000, in: The 11th Cambridge Workshop on Cool Stars, Stellar Systems, and the Sun, García López R., Rebolo R., Zapatero Osorio M.R. (eds.), ASP Conf. Ser. (in press)

Pallavicini R., Cerruti-Sola M., Duncan D.K., 1987, A\&A 174, 116
Pasquini L., Pallavicini R., Pakull M., 1988, A\&A 191, 253

Pounds K.A., Allan D.J., Barber C., et al., 1993, MNRAS 260, 77

Pye J.P., McGale P.A., Allan D.J., et al., 1995, MNRAS 274, 1165

Raveendran A.V., Mekkaden M.V., Mohin S., 1982, MNRAS 199, 707

Raveendran A.V., Mohin S., 1995, A\&A 301, 788

Raveendran A.V., Mekkaden M.V., 1998, A\&A 338, 1031

Robinson R.D., Airapetian V.S., Maran S.P., Carpenter K.G., 1996, ApJ 469, 872

Saar S.H., Huovelin J., Osten R.A., Shcherbakow A.G., 1997, A\&A 326, 741

Sanz-Forcada J., Montes D., Fernández-Figueroa M.J., De Castro E., Cornide M., 1998, in ASP Conf. Ser. 154, CD1450, The Tenth Cambridge Workshop on Cool Stars, Stellar Systems, and the Sun, Donahue R.A., Bookbinder J.A. (eds.), San Francisco: ASP

Sanz-Forcada J., Montes D., Fernández-Figueroa M.J., De Castro E., Cornide M., 1999, Solar and Stellar Activity: Similarities and Differences, Butler C.J., Doyle J.G. (eds.), ASP Conf. Ser. 158, 307

Schild R.E., 1973, AJ 78, 37

Schmidt-Kaler T., 1982, in Landolt-Börnstein, Vol. 2b, Schaifers K., Voig H.H. (eds.). Heidelberg: Springer

Smith G.H., Dupree A.K., 1988, AJ 95, 1547

Soderblom D.R., Pilachowski C.A., Fedele S.B., Jones B.F., 1990, ApJS 72, 191

Stauffer J.R., Hartmann L.W., Prosser C.F., et al., 1997, ApJ 479,776

Stawikowski A., Glebocki R., 1994, AcA 44, 393

Stencel R.E., 1978, ApJ 223, L37

Strassmeier K.G., 1989, IBVS 3290

Strassmeier K.G., 1994a, A\&A 281, 395

Strassmeier K.G., 1994b, A\&AS 103, 413

Strassmeier K.G., 1997, A\&A 319, 535

Strassmeier K.G., Hall D.S., Boyd L.J., Genet R.M., 1989a, ApJS 69, 141

Strassmeier K.G., Hotten J.T., Hall D.S., Fekel F.C., 1989b, PASP 101, 107

Strassmeier K.G., Fekel F.C., 1990, A\&A 320, 389

Strassmeier K.G., Fekel F.C., Bopp B.W., Dempsey R.C., Henry G.W., 1990, ApJS 72, 191

Strassmeier K.G., Hall D.S., Fekel F.C., Scheck M., 1993, A\&AS 100, 173 (CABS)

Strassmeier K.G., Handler G., Paunzen E., Rauth M., 1994, A\&A 281, 855

Vilhu O., Gustafsson B., Walter F.M., 1991, A\&A 241, 167

Walter F.M., Byrne P.B., 1998, in ASP Conf. Ser. 154, CD1458, The Tenth Cambridge Workshop on Cool Stars, Stellar Systems, and the Sun, Donahue R.A., Bookbinder J.A. (eds.), San Francisco: ASP

Walter F.M., 1999, in ASP Conf. Ser. 158, 87, Solar and Stellar Activity: Similarities and Differences, Butler C.J., Doyle J.G. (eds.), San Francisco: ASP

Weber M., Strassmeier K.G., 1998, A\&A 330, 1029

Wood B.E., Harper G.M., Linsky J.L., Dempsey R.C., 1996, ApJ 458, 761

Wood B.E., Linsky J.L., Ayres T.R., 1997, ApJ 478, 745

Zhang X.B., Zhang R.X., 1999, A\&AS 137, 217 


\section{List of objects}

"UX Ari" on page 104

"12 Cam" on page 104

"V1149 Ori" on page 104

"OU Gem" on page 104

" $\sigma$ Gem" on page 104

"BF Lyn" on page 104

"IL Hya" on page 104

"FG UMa" on page 104

"LR Hya" on page 104

"HU Vir" on page 104

"DK Dra" on page 104

"BQ CVn" on page 104

"IS Vir" on page 104

"BL CVn" on page 104

"BM CVn" on page 104

"MS Ser" on page 104

" $\beta$ Gem" on page 104

" $\alpha$ Tau" on page 104

"6 Lyn" on page 104

" $\alpha$ Boo" on page 104

"54 Psc" on page 104

"HR 1614" on page 104 